\title{
Relations for optical indicatrix parameters in the conditions of crystal torsion
}

\author{
Skab I., Vasylkiv Yu., Savaryn V. and Vlokh R. \\ Institute of Physical Optics, 23 Dragomanov St., 79005 Lviv, Ukraine, \\ e-mail: vlokh@ifo.lviv.ua
}

Received: 06.08.2010

\begin{abstract}
We have derived the relations describing optical indicatrix changes appearing in crystals of all the point symmetry groups for the different geometries of application of torque moment and light propagation directions.
\end{abstract}

Keywords: piezooptic effect, torsion stress

PACS: $78.20 . \mathrm{Hp}, 07.10 . \mathrm{Lw}$

UDC: 535.551

\section{Introduction}

It is known that the piezooptic effect consists in the changes of optical impermeability coefficients $\Delta B_{i j}$ (or the refractive indices $B_{i j}=\left(1 / n^{2}\right)_{i j}$ ) of a medium under the action of mechanical stress $\sigma_{k l}$. This can be described by the relation

$$
\Delta B_{i j}=B_{i j}-B_{i j}^{0}=\pi_{i j k l} \sigma_{k l},
$$

where $\pi_{i j k l}$ is the fourth-rank piezooptic tensor, and $B_{i j}$ and $B_{i j}^{0}$ the impermeability tensors of a strained and free samples, respectively.

There are many techniques aimed at study of the piezooptic effect in crystals [1]. However, in order to determine some of the piezooptic coefficients $\pi_{\lambda \mu}$ with the indices $\lambda=1,2, \ldots 6$ and $\mu=4,5,6$ in the matrix notation, one needs to apply so-called shear stress to a crystalline sample. Usually the shear-stressed state is created when loading a sample along the bisector of two mutually orthogonal crystallographic axes. When the above stress is applied, the existing components of the stress tensor are not limited to the shear ones only, and additional compressive and extension stresses appear along the principal crystallographic directions [1-3].

Besides (see, e.g., the analysis [4]), the piezooptic coefficients are usually measured with a high error that can exceed 30 per cent. This error is caused by a so-called barrelshaped distortion appearing due to a friction force between sample faces, a cover cap and a substrate used for sample loading. As a consequence, a resulting distribution of stresses inside a sample is a priori unknown. For more precise determination of the piezooptic coefficients, a three-point bending method is often used [5]. Then the stress distribution inside a sample can be determined in advance. 
Notice that the same should be true when a torsion mechanical moment is applied to a sample. Moreover, application of this kind of stresses should have the advantage consisting in possibilities for determining the shear stress-associated piezooptic coefficients. As mentioned above, the piezooptic tensor components $\pi_{\lambda \mu}$ with $\lambda=1,2, \ldots 6$ and $\mu=4,5,6$ in the matrix notation are referred to such the coefficients. Usually the latter cannot be measured in any simple way, due to a complicated experimental geometry required, and are therefore recalculated from the indirect experimental data on the basis of very cumbersome relations [2, 3], thus imposing increasing errors that can exceed the typical mean values of the coefficients themselves.

As shown in our works [6-10], application of the torsion $[6,7,9,10]$ or bending $[7$, $8,10]$ stresses leads to some spatial distribution of the optical birefringence and the angle of optical indicatrix rotation in crystals. In particular, when a crystal is twisted around $Z$ axis, a special point of zero induced birefringence is observed in the geometrical centre of $X Y$ cross section of a sample, corresponding to the zero shear stress components $\sigma_{13}$ and $\sigma_{23}$. This point belongs to the torsion axis. It has been found in our earlier studies that the birefringence linearly increases with increasing distance from the geometrical centre of the $X Y$ cross section. Moreover, it has been shown that the birefringence distribution forms a conical surface in the coordinates $(X, Y, \Delta n)[6,7]$.

The experiments mentioned above have used a single laser-beam polarimetry method, with scanning the beam across the $X Y$ face of a sample. This method reveals a low resolution limited by the laser beam diameter and so should be successfully replaced by an imaging polarimetric technique.

If a cylindrical sample is twisted around the $Z$ axis, the relevant stress tensor components may be determined as [11]

$$
\sigma_{\mu}=\frac{2 M_{z}}{\pi R^{4}}\left(X \delta_{4 \mu}-Y \delta_{5 \mu}\right),
$$

where $M_{z}=\int_{S} r \times P d S, \delta_{4 \mu}$ and $\delta_{5 \mu}$ are the Kronecker deltas, $R$ the cylinder radius, $S$ the square of the cylinder basis, and $P$ the mechanical load. Thus, we deal with the two shear components of the stress tensor, $\sigma_{32}$ and $\sigma_{31}$ :

$$
\sigma_{4}=\sigma_{23}=\frac{2 M_{z}}{\pi R^{4}} X
$$

and

$$
\sigma_{5}=\sigma_{13}=\frac{2 M_{z}}{\pi R^{4}} Y,
$$

which linearly depend on the coordinates. This dependence enables one to determine unambiguously a distribution of shear stress components inside a sample under study. Furthermore, application of the torsion moment makes it possible to produce pure tangential displacements (i.e., the pure shear stress components), which are usually 


\begin{tabular}{|c|c|c|c|c|c|c|c|c|c|}
\hline 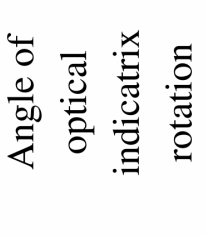 & 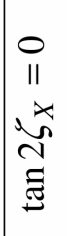 & 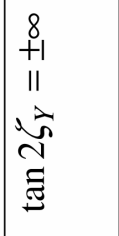 & $\begin{array}{l}8 \\
+1 \\
11 \\
1 \\
N \\
N \\
N \\
\Xi \\
\Xi\end{array}$ & 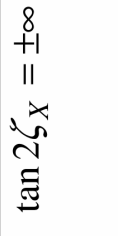 & 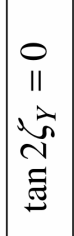 & 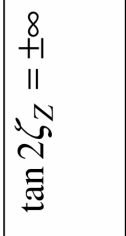 & 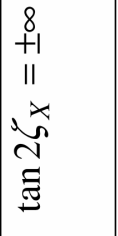 & 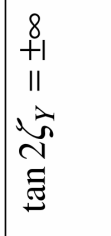 & 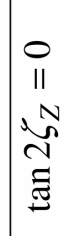 \\
\hline 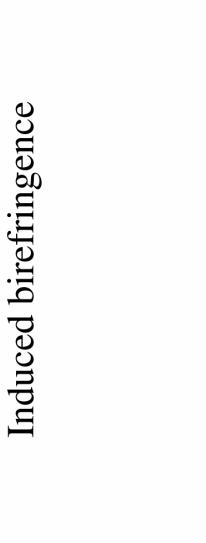 & $\begin{array}{l}0 \\
11 \\
3 \\
y^{3}\end{array}$ & 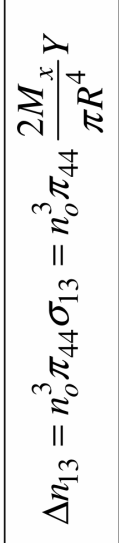 & 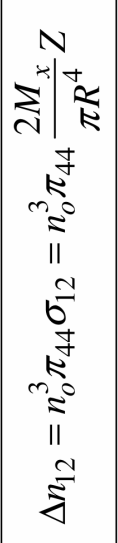 & 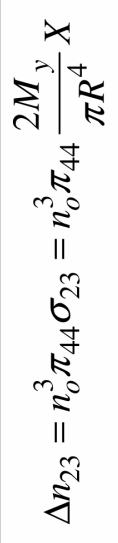 & \begin{tabular}{c}
0 \\
11 \\
$m$ \\
\multirow{2}{z}{}
\end{tabular} & 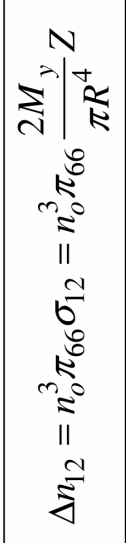 & 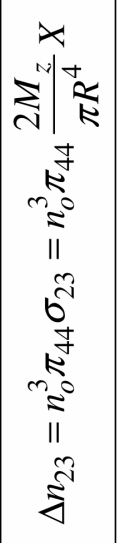 & 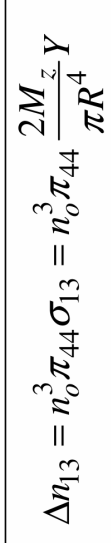 & $\begin{array}{c}2 \\
3 \\
11 \\
0 \\
0 \\
2\end{array}$ \\
\hline 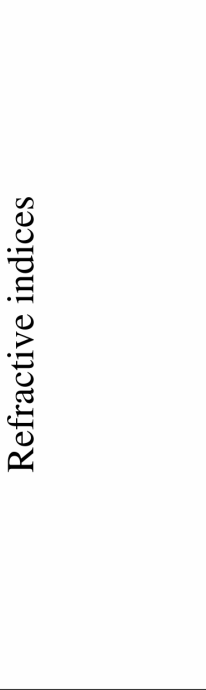 & 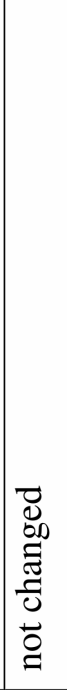 & 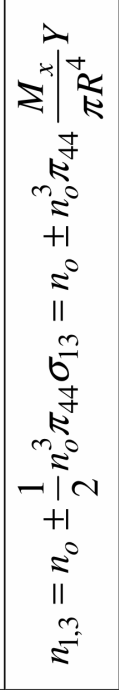 & 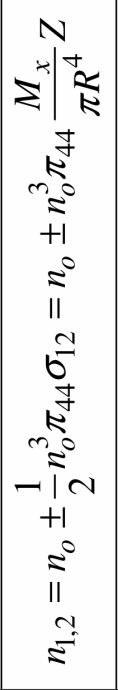 & 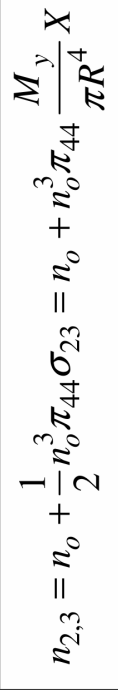 & 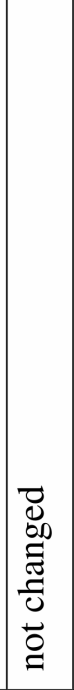 & 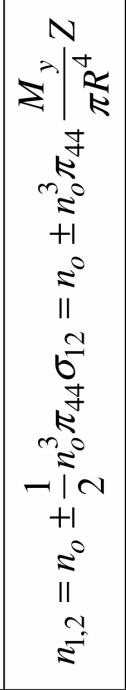 & 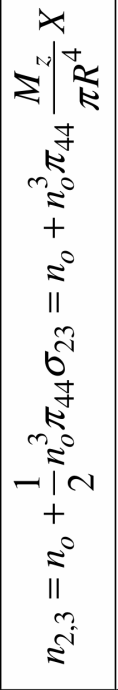 & 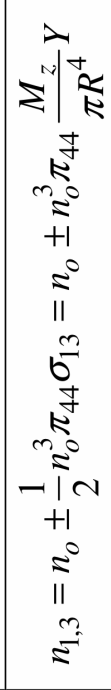 & 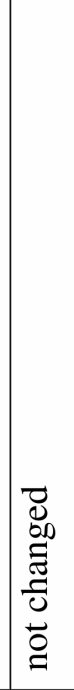 \\
\hline 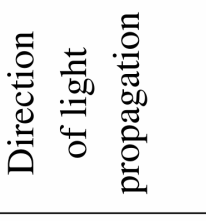 & $\stackrel{\nexists}{\overline{\#}}$ & $\frac{\lambda}{\equiv}$ & $\frac{N}{\underline{N}}$ & $\frac{\gtrsim}{\overline{\#}}$ & $\stackrel{\lambda}{\underline{\underline{N}}}$ & $\stackrel{N}{\equiv}$ & $\frac{\infty}{\underline{N}}$ & $\stackrel{ }{\equiv}$ & $\frac{N}{\underline{N}}$ \\
\hline 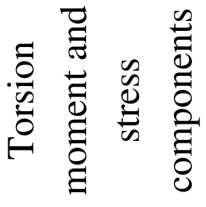 & 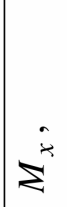 & $\begin{array}{l}\hat{b}^{2} \\
\hat{i}\end{array}$ & & $\approx \hat{b}$ & & & $\sum^{n} \quad \dot{b}$ & & \\
\hline
\end{tabular}




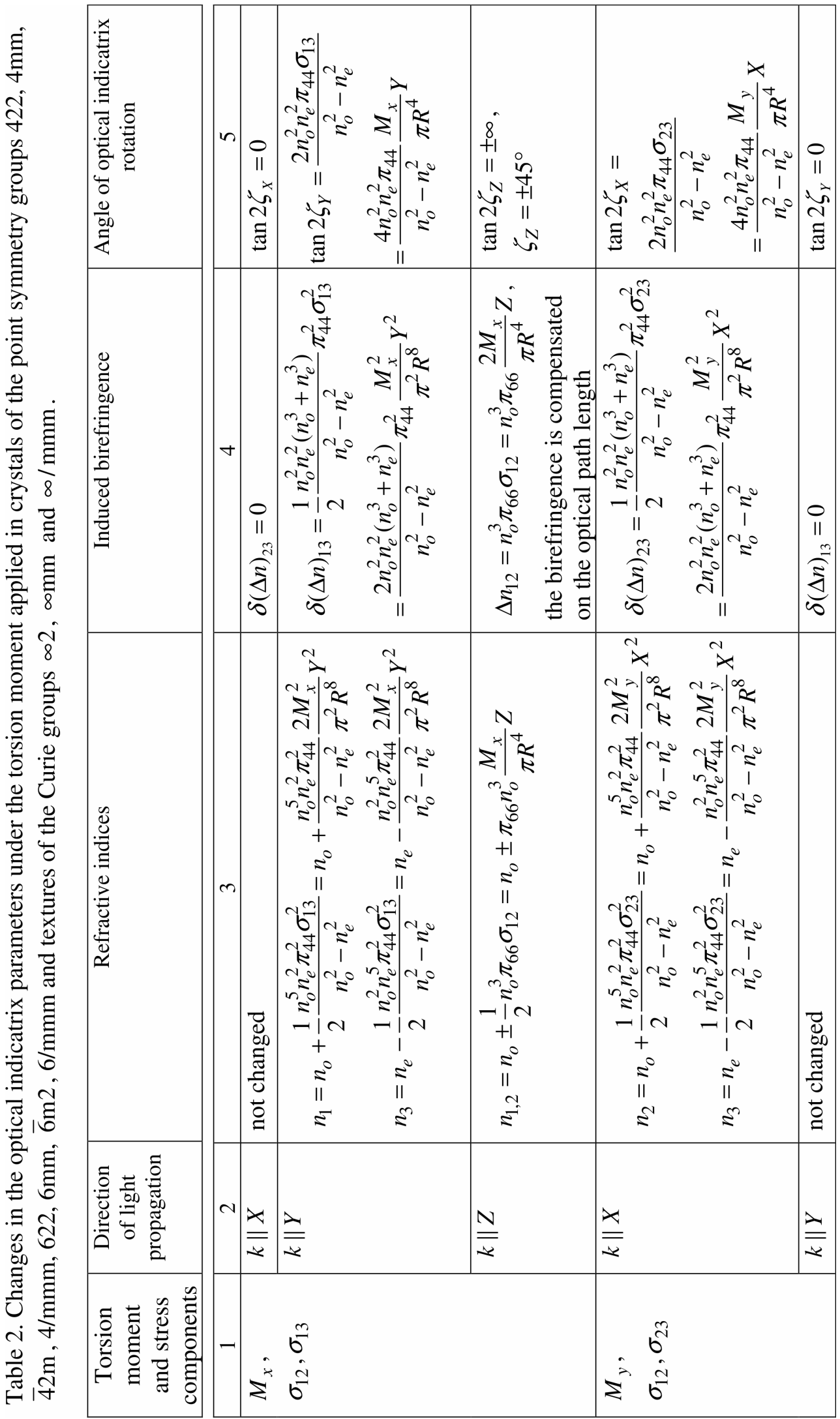




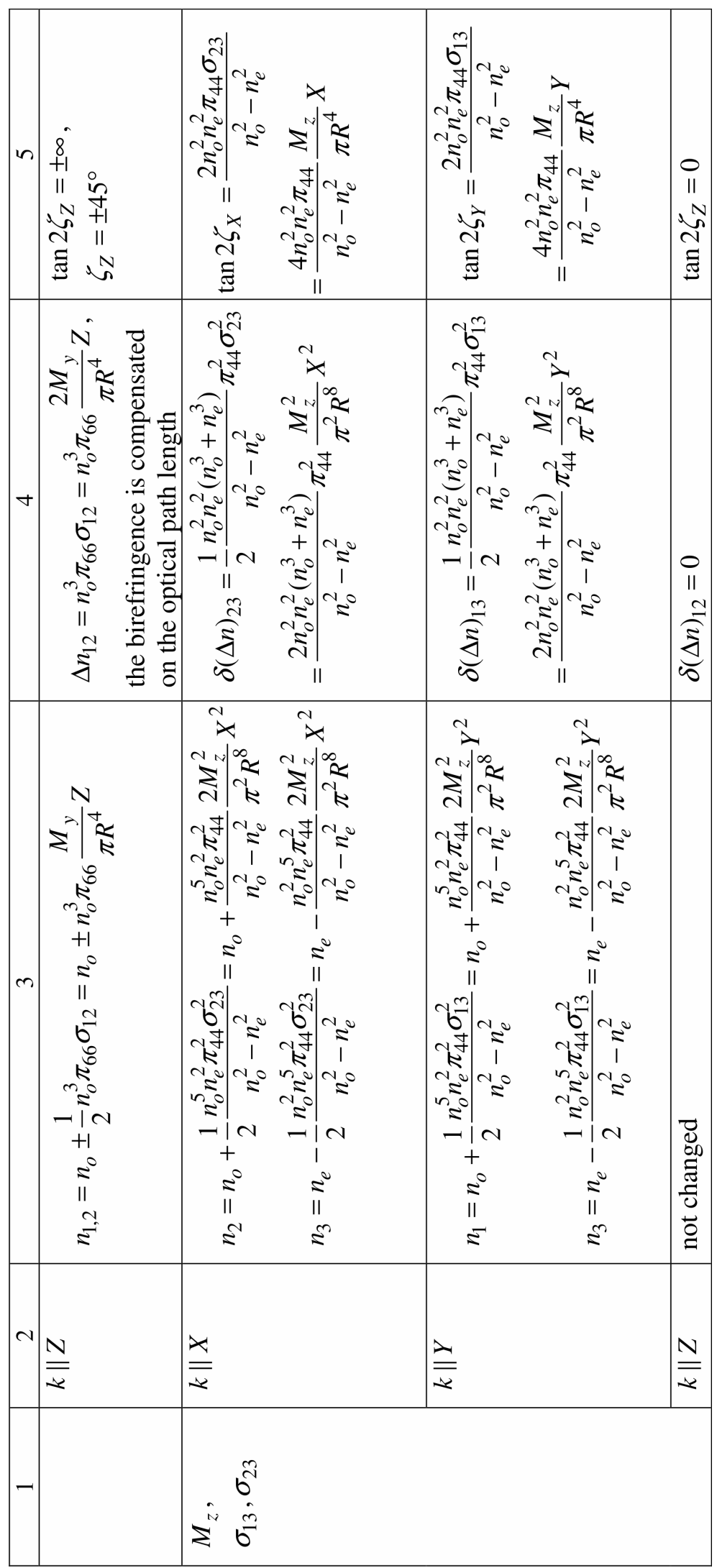

Ukr. J. Phys. Opt. 2010, V11, №4 
Table 3. Changes in the optical indicatrix parameters under the torsion moment applied in crystals of the point symmetry groups $6, \overline{6}, 6 / \mathrm{m}$ and textures of the Curie groups $\infty$ and $\infty / m$.

\section{Torsion $\quad$ Direction of}

moment and

stress

light

Refractive indices

components

\begin{tabular}{|c|c|c|}
\hline 1 & 2 & 3 \\
\hline \multirow[t]{3}{*}{$\begin{array}{l}M_{x}, \\
\sigma_{12}, \sigma_{13}\end{array}$} & $k \| X$ & $\begin{array}{l}n_{2}=n_{o}+\frac{n_{o}^{3}}{2}\left(2 \pi_{62} \sigma_{12}+\frac{\pi_{45}^{2} \sigma_{13}^{2} n_{o}^{2} n_{e}^{2}}{n_{o}^{2}-n_{e}^{2}+2 n_{o}^{2} n_{e}^{2} \pi_{62} \sigma_{12}}\right) \\
=n_{o}+2 n_{o}^{3}\left(\frac{\pi_{62} M_{x}}{\pi R^{4}} Z+\frac{n_{o}^{2} n_{e}^{2} \pi_{45}^{2} \frac{M_{x}^{2}}{\pi^{2} R^{8}} Y^{2}}{n_{o}^{2}-n_{e}^{2}+4 n_{o}^{2} n_{e}^{2} \pi_{62} \frac{M_{x}}{\pi R^{4}} Z}\right) \\
n_{3}=n_{e}-\frac{n_{e}^{3}}{2} \frac{\pi_{45}^{2} \sigma_{13}^{2} n_{o}^{2} n_{e}^{2}}{n_{o}^{2}-n_{e}^{2}+2 n_{o}^{2} n_{e}^{2} \pi_{62} \sigma_{12}} \\
=n_{e}-2 n_{e}^{3} \frac{n_{o}^{2} n_{e}^{2} \pi_{45}^{2} \frac{M_{x}^{2}}{\pi^{2} R^{8}} Y^{2}}{n_{o}^{2}-n_{e}^{2}+4 n_{o}^{2} n_{e}^{2} \pi_{62} \frac{M_{x}}{\pi R^{4}} Z}\end{array}$ \\
\hline & $k \| Y$ & $\begin{array}{l}n_{1}=n_{o}-\frac{n_{o}^{3}}{2}\left(2 \pi_{62} \sigma_{12}-\frac{n_{o}^{2} n_{e}^{2} \pi_{44}^{2} \sigma_{13}^{2}}{n_{o}^{2}-n_{e}^{2}-2 n_{o}^{2} n_{e}^{2} \pi_{62} \sigma_{12}}\right) \\
=n_{0}-2 n_{o}^{3}\left(\frac{\pi_{62} M_{x}}{\pi R^{4}} Z-\frac{n_{o}^{2} n_{e}^{2} \pi_{44}^{2} \frac{M_{x}^{2}}{\pi^{2} R^{8}} Y^{2}}{n_{o}^{2}-n_{e}^{2}-2 n_{o}^{2} n_{e}^{2} \pi_{62} \frac{2 M_{x}}{\pi R^{4}} Z}\right) \\
n_{3}=n_{e}-\frac{n_{e}^{3}}{2} \frac{n_{o}^{2} n_{e}^{2} \pi_{44}^{2} \sigma_{13}^{2}}{n_{o}^{2}-n_{e}^{2}-2 n_{o}^{2} n_{e}^{2} \pi_{62} \sigma_{12}} \\
=n_{e}-2 n_{e}^{3} \frac{n_{o}^{2} n_{e}^{2} \pi_{44}^{2} \frac{M_{x}^{2}}{\pi^{2} R^{8}} Y^{2}}{n_{o}^{2}-n_{e}^{2}-2 n_{o}^{2} n_{e}^{2} \pi_{62} \frac{2 M_{x}}{\pi R^{4}} Z}\end{array}$ \\
\hline & $k \| Z$ & $\begin{array}{l}n_{1}=n_{o}-\frac{n_{o}^{3}}{2} \sigma_{12} \sqrt{4 \pi_{62}^{2}+\pi_{66}^{2}}=n_{o}-n_{o}^{3} \frac{M_{x}}{\pi R^{4}} Z \sqrt{4 \pi_{62}^{2}+\pi_{66}^{2}} \\
n_{2}=n_{o}+\frac{n_{o}^{3}}{2} \sigma_{12} \sqrt{4 \pi_{62}^{2}+\pi_{66}^{2}}=n_{o}+n_{o}^{3} \frac{M_{x}}{\pi R^{4}} Z \sqrt{4 \pi_{62}^{2}+\pi_{66}^{2}}\end{array}$ \\
\hline
\end{tabular}




\begin{tabular}{|c|c|}
\hline Induced birefringence & $\begin{array}{c}\text { Angle of optical indicatrix } \\
\text { rotation }\end{array}$ \\
\hline 4 & 5 \\
\hline $\begin{array}{l}\delta(\Delta n)_{23}=n_{o}^{3} \pi_{62} \sigma_{12}+\frac{1}{2} \frac{\left(n_{o}^{3}+n_{e}^{3}\right) \pi_{45}^{2} \sigma_{13}^{2} n_{o}^{2} n_{e}^{2}}{n_{o}^{2}-n_{e}^{2}+2 n_{o}^{2} n_{e}^{2} \pi_{62} \sigma_{12}} \\
=2 n_{o}^{3} \pi_{62} \frac{M_{x}}{\pi R^{4}} Z+2 \frac{\left(n_{o}^{3}+n_{e}^{3}\right) n_{o}^{2} n_{e}^{2} \pi_{45}^{2} \frac{M_{x}^{2}}{\pi^{2} R^{8}} Y^{2}}{n_{o}^{2}-n_{e}^{2}+4 n_{o}^{2} n_{e}^{2} \pi_{62} \frac{M_{x}}{\pi R^{4}} Z} \\
\simeq n_{o}^{3} \pi_{62} \sigma_{12}=2 n_{o}^{3} \pi_{62} \frac{M_{x}}{\pi R^{4}} Z\end{array}$ & $\begin{array}{l}\tan 2 \zeta_{X}= \\
=\frac{2 \pi_{45} \sigma_{13} n_{o}^{2} n_{e}^{2}}{n_{o}^{2}-n_{e}^{2}+2 n_{o}^{2} n_{e}^{2} \pi_{62} \sigma_{12}} \\
=\frac{4 n_{o}^{2} n_{e}^{2} \pi_{45} \frac{M_{x}}{\pi R^{4}} Y}{n_{o}^{2}-n_{e}^{2}+4 n_{o}^{2} n_{e}^{2} \pi_{62} \frac{M_{x}}{\pi R^{4}} Z}\end{array}$ \\
\hline $\begin{array}{l}\delta(\Delta n)_{13}=n_{o}^{3} \pi_{62} \sigma_{12}-\frac{1}{2} \frac{\left(n_{o}^{3}+n_{e}^{3}\right) n_{o}^{2} n_{e}^{2} \pi_{44}^{2} \sigma_{13}^{2}}{n_{o}^{2}-n_{e}^{2}-2 n_{o}^{2} n_{e}^{2} \pi_{62} \sigma_{12}} \\
=n_{o}^{3} \pi_{62} \frac{2 M_{x}}{\pi R^{4}} Z-2 \frac{\left(n_{o}^{3}+n_{e}^{3}\right) n_{o}^{2} n_{e}^{2} \pi_{44}^{2} \frac{M_{x}^{2}}{\pi^{2} R^{8}} Y^{2}}{n_{o}^{2}-n_{e}^{2}-4 n_{o}^{2} n_{e}^{2} \pi_{62} \frac{M_{x}}{\pi R^{4}} Z} \\
\simeq n_{o}^{3} \pi_{62} \sigma_{12}=n_{o}^{3} \pi_{62} \frac{2 M_{x}}{\pi R^{4}} Z\end{array}$ & $\begin{array}{l}\tan 2 \zeta_{Y}= \\
=\frac{2 n_{o}^{2} n_{e}^{2} \pi_{44} \sigma_{13}}{n_{o}^{2}-n_{e}^{2}-2 n_{o}^{2} n_{e}^{2} \pi_{62} \sigma_{12}} \\
=\frac{4 n_{o}^{2} n_{e}^{2} \pi_{44} \frac{M_{x}}{\pi R^{4}} Y}{n_{o}^{2}-n_{e}^{2}-4 n_{o}^{2} n_{e}^{2} \pi_{62} \frac{M_{x}}{\pi R^{4}} Z}\end{array}$ \\
\hline $\begin{array}{l}\delta(\Delta n)_{12}=n_{o}^{3} \sigma_{12} \sqrt{4 \pi_{62}^{2}+\pi_{66}^{2}} \\
=2 n_{o}^{3} \frac{M_{x}}{\pi R^{4}} Z \sqrt{4 \pi_{62}^{2}+\pi_{66}^{2}} \\
\text { the birefringence is compensated on the optical path } \\
\text { length }\end{array}$ & $\tan 2 \zeta_{Z}=\frac{\pi_{66}}{2 \pi_{62}}$ \\
\hline
\end{tabular}




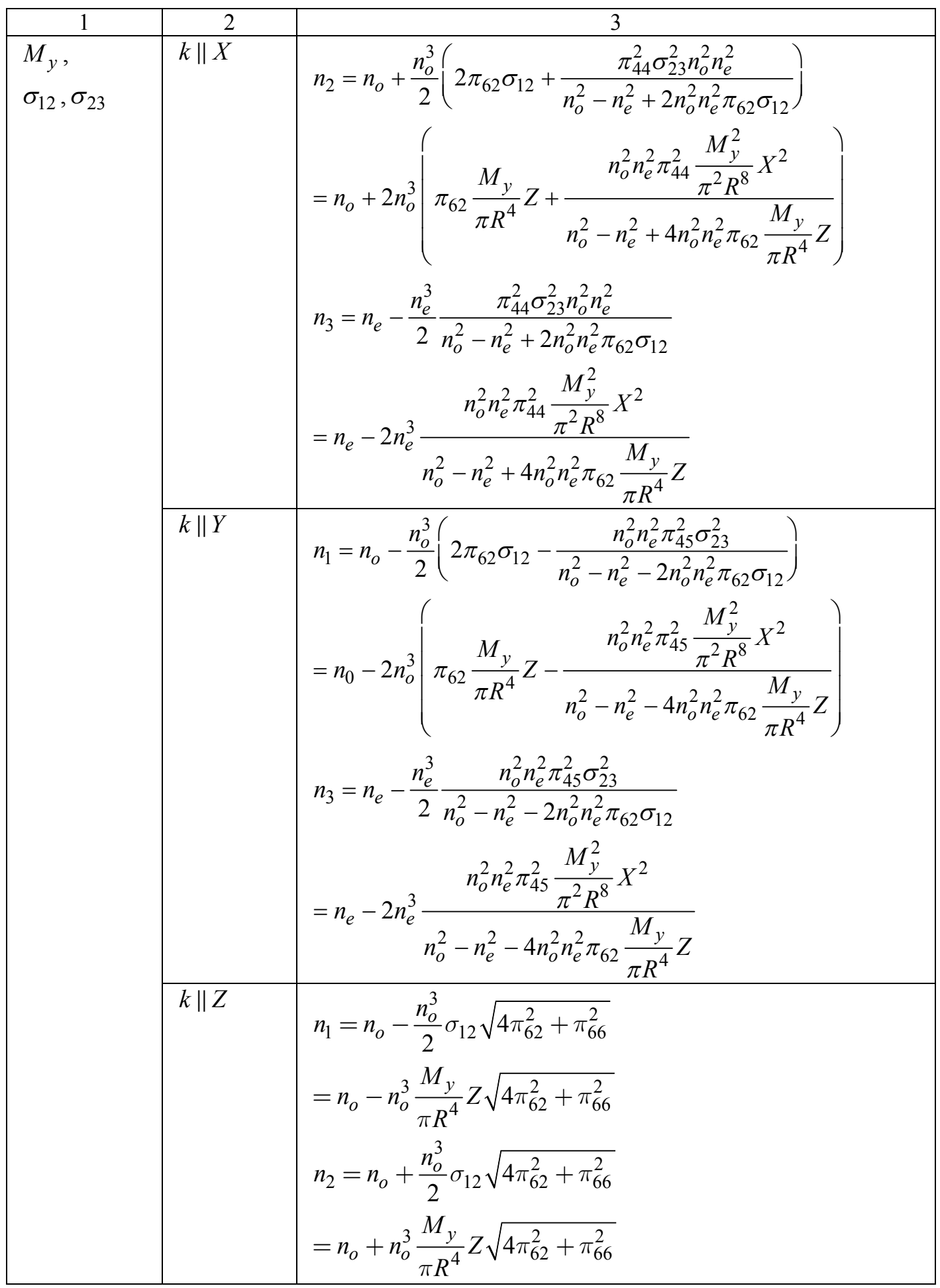




\begin{tabular}{|c|c|}
\hline 4 & 5 \\
\hline $\begin{array}{l}\delta(\Delta n)_{23}=n_{o}^{3} \pi_{62} \sigma_{12}+\frac{1}{2} \frac{\left(n_{o}^{3}+n_{e}^{3}\right) \pi_{44}^{2} \sigma_{23}^{2} n_{o}^{2} n_{e}^{2}}{n_{o}^{2}-n_{e}^{2}+2 n_{o}^{2} n_{e}^{2} \pi_{62} \sigma_{12}} \\
=2 n_{o}^{3} \frac{\pi_{62} M_{y}}{\pi R^{4}} Z+2 \frac{\left(n_{o}^{3}+n_{e}^{3}\right) n_{o}^{2} n_{e}^{2} \pi_{44}^{2} \frac{M_{y}^{2}}{\pi^{2} R^{8}} X^{2}}{n_{o}^{2}-n_{e}^{2}+4 n_{o}^{2} n_{e}^{2} \pi_{62} \frac{M_{y}}{\pi R^{4}} Z} \\
\simeq n_{o}^{3} \pi_{62} \sigma_{12}=2 n_{o}^{3} \pi_{62} \frac{M_{y}}{\pi R^{4}} Z\end{array}$ & $\begin{array}{l}\tan 2 \zeta_{X}=\frac{2 \pi_{44} \sigma_{23} n_{o}^{2} n_{e}^{2}}{n_{o}^{2}-n_{e}^{2}+2 n_{o}^{2} n_{e}^{2} \pi_{62} \sigma_{12}} \\
=\frac{4 n_{o}^{2} n_{e}^{2} \pi_{44} \frac{M_{y}}{\pi R^{4}} X}{n_{o}^{2}-n_{e}^{2}+4 n_{o}^{2} n_{e}^{2} \pi_{62} \frac{M_{y}}{\pi R^{4}} Z}\end{array}$ \\
\hline $\begin{array}{l}\delta(\Delta n)_{13}=n_{o}^{3} \pi_{62} \sigma_{12}-\frac{1}{2} \frac{\left(n_{o}^{3}+n_{e}^{3}\right) n_{o}^{2} n_{e}^{2} \pi_{45}^{2} \sigma_{23}^{2}}{n_{o}^{2}-n_{e}^{2}-2 n_{o}^{2} n_{e}^{2} \pi_{62} \sigma_{12}} \\
=n_{o}^{3} \frac{2 \pi_{62} M_{y}}{\pi R^{4}} Z-\frac{\left(n_{o}^{3}+n_{e}^{3}\right) n_{o}^{2} n_{e}^{2} \pi_{45}^{2} \frac{M_{y}^{2}}{\pi^{2} R^{8}} X^{2}}{n_{o}^{2}-n_{e}^{2}-4 n_{o}^{2} n_{e}^{2} \pi_{62} \frac{M_{y}}{\pi R^{4}} Z} \\
\simeq n_{o}^{3} \pi_{62} \sigma_{12}=n_{o}^{3} \pi_{62} \frac{2 M_{y}}{\pi R^{4}} Z\end{array}$ & $\begin{array}{l}\tan 2 \zeta_{Y}=\frac{n_{o}^{2} n_{e}^{2} \pi_{45} \sigma_{23}}{n_{o}^{2}-n_{e}^{2}-2 n_{o}^{2} n_{e}^{2} \pi_{62} \sigma_{12}} \\
=\frac{4 n_{o}^{2} n_{e}^{2} \pi_{45} \frac{M_{y}}{\pi R^{4}} X}{n_{o}^{2}-n_{e}^{2}-4 n_{o}^{2} n_{e}^{2} \pi_{62} \frac{M_{y}}{\pi R^{4}} Z}\end{array}$ \\
\hline $\begin{array}{l}\delta(\Delta n)_{12}=n_{o}^{3} \sigma_{12} \sqrt{4 \pi_{62}^{2}+\pi_{66}^{2}} \\
=2 n_{o}^{3} \frac{M_{y}}{\pi R^{4}} Z \sqrt{4 \pi_{62}^{2}+\pi_{66}^{2}} \\
\text { the birefringence is compensated on the optical } \\
\text { path length }\end{array}$ & $\tan 2 \zeta_{Z}=\frac{\pi_{66}}{2 \pi_{62}}$ \\
\hline
\end{tabular}




\begin{tabular}{|c|c|c|}
\hline 1 & 2 & 3 \\
\hline \multirow[t]{3}{*}{$\begin{array}{l}M_{z}, \\
\sigma_{13}, \sigma_{23}\end{array}$} & $k \| X$ & $\begin{array}{l}n_{2}=n_{o}-\frac{1}{2} n_{o}^{3} \frac{n_{o}^{2} n_{e}^{2}\left(\pi_{44} \sigma_{23}+\pi_{45} \sigma_{13}\right)^{2}}{n_{o}^{2}-n_{e}^{2}} \\
=n_{o}-2 n_{o}^{3} \frac{n_{o}^{2} n_{e}^{2} \frac{M_{z}^{2}}{\pi^{2} R^{8}}\left(\pi_{44} X+\pi_{45} Y\right)^{2}}{n_{o}^{2}-n_{e}^{2}} \\
n_{3}=n_{e}+\frac{1}{2} n_{e}^{3} \frac{n_{o}^{2} n_{e}^{2}\left(\pi_{44} \sigma_{23}+\pi_{45} \sigma_{13}\right)^{2}}{n_{o}^{2}-n_{e}^{2}} \\
=n_{e}+2 n_{e}^{3} \frac{n_{o}^{2} n_{e}^{2} \frac{M_{z}^{2}}{\pi^{2} R^{8}}\left(\pi_{44} X+\pi_{45} Y\right)^{2}}{n_{o}^{2}-n_{e}^{2}}\end{array}$ \\
\hline & $k \| Y$ & $\begin{array}{l}n_{1}=n_{o}-\frac{1}{2} n_{o}^{3} \frac{n_{o}^{2} n_{e}^{2}\left(\pi_{44} \sigma_{13}-\pi_{45} \sigma_{23}\right)^{2}}{n_{o}^{2}-n_{e}^{2}} \\
=n_{o}-2 n_{o}^{3} \frac{n_{o}^{2} n_{e}^{2} \frac{M_{z}^{2}}{\pi^{2} R^{8}}\left(\pi_{44} Y-\pi_{45} X\right)^{2}}{n_{o}^{2}-n_{e}^{2}} \\
n_{3}=n_{e}+\frac{1}{2} n_{e}^{3} \frac{n_{o}^{2} n_{e}^{2}\left(\pi_{44} \sigma_{13}-\pi_{45} \sigma_{23}\right)^{2}}{n_{o}^{2}-n_{e}^{2}} \\
=n_{e}+2 n_{e}^{3} \frac{n_{o}^{2} n_{e}^{2} \frac{M_{z}^{2}}{\pi^{2} R^{8}}\left(\pi_{44} Y-\pi_{45} X\right)^{2}}{n_{o}^{2}-n_{e}^{2}}\end{array}$ \\
\hline & $k \| Z$ & not changed \\
\hline
\end{tabular}




\begin{tabular}{|c|c|}
\hline 4 & 5 \\
\hline $\begin{array}{l}\delta(\Delta n)_{23}=\frac{1}{2}\left(n_{o}^{3}+n_{e}^{3}\right) \frac{n_{o}^{2} n_{e}^{2}\left(\pi_{44} \sigma_{23}+\pi_{45} \sigma_{13}\right)^{2}}{n_{o}^{2}-n_{e}^{2}} \\
=2\left(n_{o}^{3}+n_{e}^{3}\right) \frac{n_{o}^{2} n_{e}^{2} \frac{M_{z}^{2}}{\pi^{2} R^{8}}\left(\pi_{44} X+\pi_{45} Y\right)^{2}}{n_{o}^{2}-n_{e}^{2}}\end{array}$ & $\begin{array}{l}\tan 2 \zeta_{X}= \\
=\frac{2 n_{o}^{2} n_{e}^{2}\left(\pi_{44} \sigma_{23}+\pi_{45} \sigma_{13}\right)}{n_{o}^{2}-n_{e}^{2}} \\
=\frac{4 n_{o}^{2} n_{e}^{2} \frac{M_{z}}{\pi R^{4}}\left(\pi_{44} X+\pi_{45} Y\right)}{n_{o}^{2}-n_{e}^{2}}\end{array}$ \\
\hline $\begin{array}{l}\delta(\Delta n)_{13}=\frac{1}{2}\left(n_{o}^{3}+n_{e}^{3}\right) \frac{n_{o}^{2} n_{e}^{2}\left(\pi_{44} \sigma_{13}+\pi_{45} \sigma_{23}\right)^{2}}{n_{o}^{2}-n_{e}^{2}} \\
=2\left(n_{o}^{3}+n_{e}^{3}\right) \frac{n_{o}^{2} n_{e}^{2} \frac{M_{z}^{2}}{\pi^{2} R^{8}}\left(\pi_{44} Y+\pi_{45} X\right)^{2}}{n_{o}^{2}-n_{e}^{2}}\end{array}$ & $\begin{array}{l}\tan 2 \zeta_{Y}= \\
=\frac{2 n_{o}^{2} n_{e}^{2}\left(\pi_{44} \sigma_{13}-\pi_{45} \sigma_{23}\right)}{n_{o}^{2}-n_{e}^{2}} \\
=\frac{4 n_{o}^{2} n_{e}^{2} \frac{M_{z}}{\pi R^{4}}\left(\pi_{44} Y-\pi_{45} X\right)}{n_{o}^{2}-n_{e}^{2}}\end{array}$ \\
\hline$\delta(\Delta n)_{12}=0$ & $\tan 2 \zeta_{Z}=0$ \\
\hline
\end{tabular}


Table 4. Changes in the optical indicatrix parameters under the torsion moment applied in crystals of the point symmetry groups $4, \overline{4}$ and $4 / \mathrm{m}$..

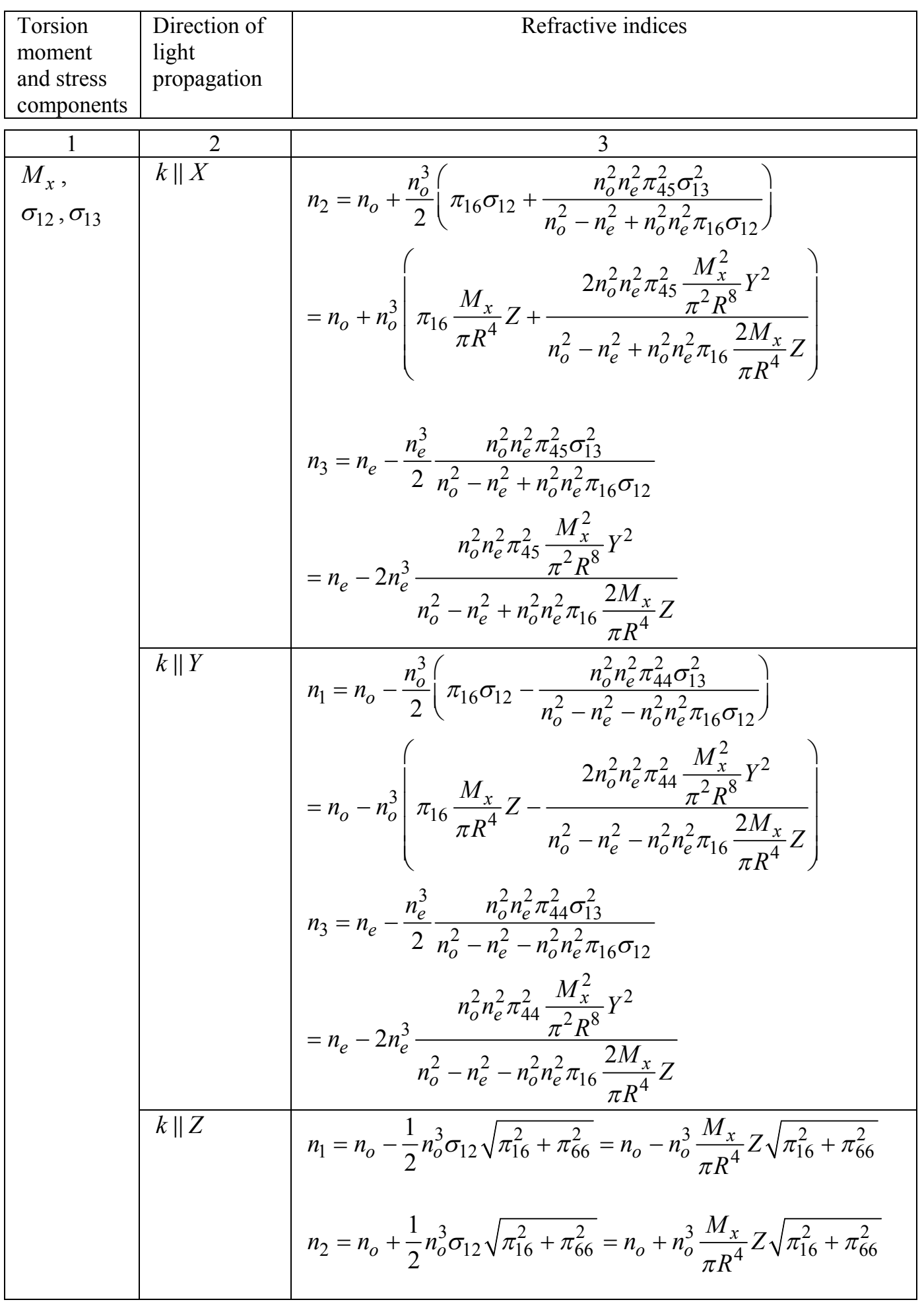




\begin{tabular}{|c|c|}
\hline Induced birefringence & Angle of optical indicatrix rotation \\
\hline 4 & 5 \\
\hline $\begin{array}{l}\delta(\Delta n)_{23}=\frac{n_{o}^{3}}{2} \pi_{16} \sigma_{12}+\frac{1}{2} \frac{\left(n_{o}^{3}+n_{e}^{3}\right) n_{o}^{2} n_{e}^{2} \pi_{45}^{2} \sigma_{13}^{2}}{n_{o}^{2}-n_{e}^{2}+n_{o}^{2} n_{e}^{2} \pi_{16} \sigma_{12}} \\
=n_{o}^{3} \pi_{16} \frac{M_{x}}{\pi R^{4}} Z+2 \frac{\left(n_{o}^{3}+n_{e}^{3}\right) n_{o}^{2} n_{e}^{2} \pi_{45}^{2} \frac{M_{x}^{2}}{\pi^{2} R^{8}} Y^{2}}{n_{o}^{2}-n_{e}^{2}+n_{o}^{2} n_{e}^{2} \pi_{16} \frac{2 M_{x}}{\pi R^{4}} Z} \\
\simeq \frac{n_{o}^{3}}{2} \pi_{16} \sigma_{12}=n_{o}^{3} \pi_{16} \frac{M_{x}}{\pi R^{4}} Z\end{array}$ & $\begin{array}{l}\tan 2 \zeta_{X}=\frac{2 n_{o}^{2} n_{e}^{2} \pi_{45} \sigma_{13}}{n_{o}^{2}-n_{e}^{2}+n_{o}^{2} n_{e}^{2} \pi_{16} \sigma_{12}} \\
=\frac{4 n_{o}^{2} n_{e}^{2} \pi_{45} \frac{M_{x}}{\pi R^{4}} Y}{n_{o}^{2}-n_{e}^{2}+n_{o}^{2} n_{e}^{2} \pi_{16} \frac{2 M_{x}}{\pi R^{4}} Z}\end{array}$ \\
\hline $\begin{array}{l}\delta(\Delta n)_{13}=\frac{n_{o}^{3}}{2} \pi_{16} \sigma_{12}-\frac{1}{2} \frac{\left(n_{o}^{3}+n_{e}^{3}\right) n_{o}^{2} n_{e}^{2} \pi_{44}^{2} \sigma_{13}^{2}}{n_{o}^{2}-n_{e}^{2}-n_{o}^{2} n_{e}^{2} \pi_{16} \sigma_{12}} \\
=n_{o}^{3} \pi_{16} \frac{M_{x}}{\pi R^{4}} Z-2 \frac{\left(n_{o}^{3}+n_{e}^{3}\right) n_{o}^{2} n_{e}^{2} \pi_{44}^{2} \frac{M_{x}^{2}}{\pi^{2} R^{8}} Y^{2}}{n_{o}^{2}-n_{e}^{2}-n_{o}^{2} n_{e}^{2} \pi_{16} \frac{2 M_{x}}{\pi R^{4}} Z} \\
\simeq \frac{n_{o}^{3}}{2} \pi_{16} \sigma_{12}=n_{o}^{3} \pi_{16} \frac{M_{x}}{\pi R^{4}} Z\end{array}$ & $\begin{array}{l}\tan 2 \zeta_{Y}=\frac{2 n_{o}^{2} n_{e}^{2} \pi_{44} \sigma_{13}}{n_{o}^{2}-n_{e}^{2}-n_{o}^{2} n_{e}^{2} \pi_{16} \sigma_{12}} \\
=\frac{4 n_{o}^{2} n_{e}^{2} \pi_{44} \frac{M_{x}}{\pi R^{4}} Y}{n_{o}^{2}-n_{e}^{2}-n_{o}^{2} n_{e}^{2} \pi_{16} \frac{2 M_{x}}{\pi R^{4}} Z}\end{array}$ \\
\hline $\begin{array}{l}\delta(\Delta n)_{12}=n_{o}^{3} \sigma_{12} \sqrt{\pi_{16}^{2}+\pi_{66}^{2}} \\
=n_{o}^{3} \frac{2 M_{x}}{\pi R^{4}} Z \sqrt{\pi_{16}^{2}+\pi_{66}^{2}} \\
\text { the birefringence is compensated on the optical } \\
\text { path length }\end{array}$ & $\tan 2 \zeta_{Z}=\frac{\pi_{66}}{\pi_{16}}$ \\
\hline
\end{tabular}


Skab I. et al

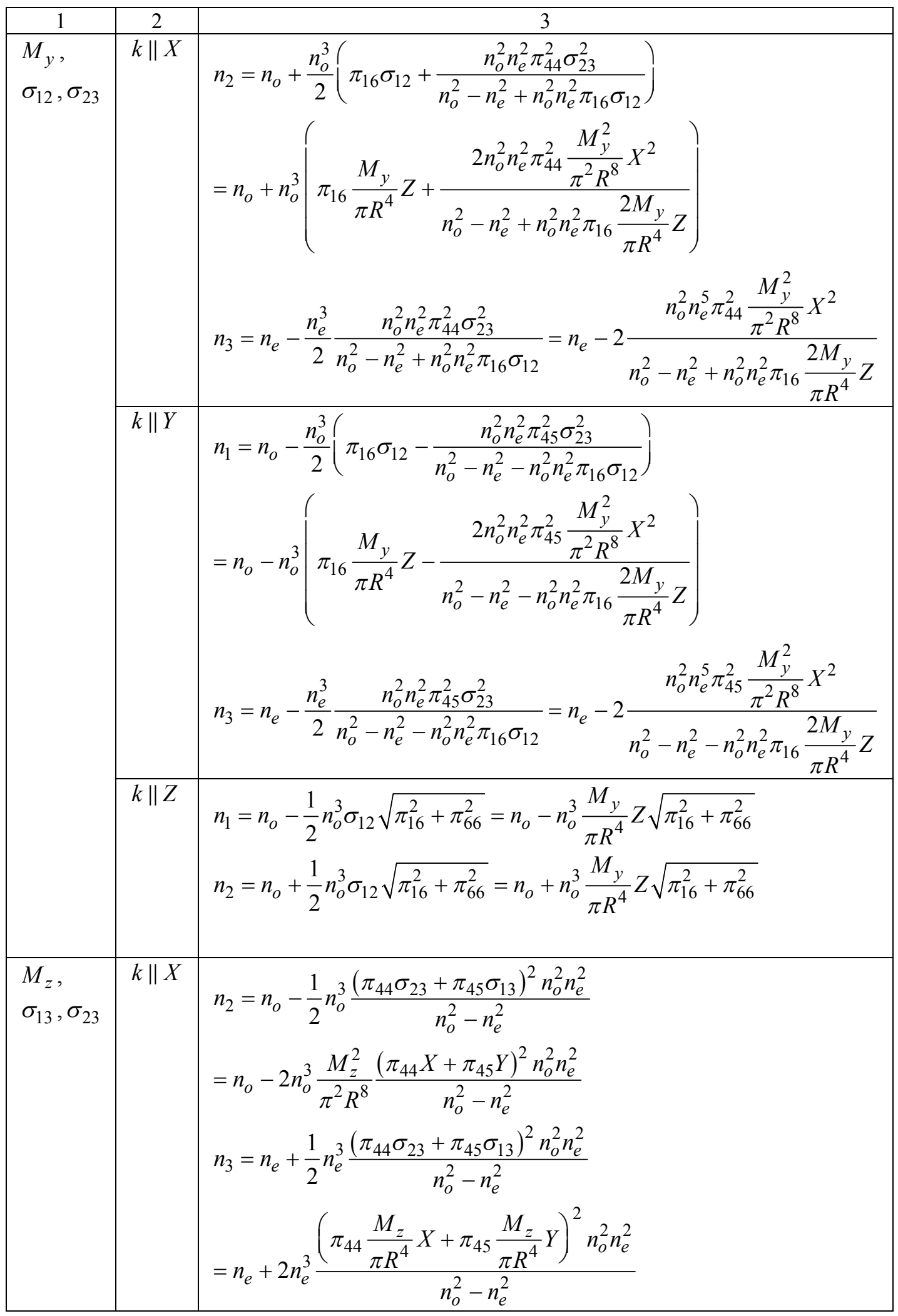

206

Ukr. J. Phys. Opt. 2010, V11, №4 


\begin{tabular}{|c|c|}
\hline 4 & 5 \\
\hline $\begin{array}{l}\delta(\Delta n)_{23}=\frac{n_{o}^{3}}{2} \pi_{16} \sigma_{12}+\frac{1}{2} \frac{\left(n_{o}^{3}+n_{e}^{3}\right) n_{o}^{2} n_{e}^{2} \pi_{44}^{2} \sigma_{23}^{2}}{n_{o}^{2}-n_{e}^{2}+n_{o}^{2} n_{e}^{2} \pi_{16} \sigma_{12}} \\
=n_{o}^{3} \pi_{16} \frac{M_{y}}{\pi R^{4}} Z+2 \frac{\left(n_{o}^{3}+n_{e}^{3}\right) n_{o}^{2} n_{e}^{2} \pi_{44}^{2} \frac{M_{y}^{2}}{\pi^{2} R^{8}} X^{2}}{n_{o}^{2}-n_{e}^{2}+n_{o}^{2} n_{e}^{2} \pi_{16} \frac{2 M_{y}}{\pi R^{4}} Z} \\
\simeq \frac{n_{o}^{3}}{2} \pi_{16} \sigma_{12}=n_{o}^{3} \pi_{16} \frac{M_{y}}{\pi R^{4}} Z\end{array}$ & $\begin{array}{l}\tan 2 \zeta_{X}=\frac{2 n_{o}^{2} n_{e}^{2} \pi_{44} \sigma_{23}}{n_{o}^{2}-n_{e}^{2}+n_{o}^{2} n_{e}^{2} \pi_{16} \sigma_{12}} \\
=\frac{4 n_{o}^{2} n_{e}^{2} \pi_{44} \frac{M_{y}}{\pi R^{4}} X}{n_{o}^{2}-n_{e}^{2}+n_{o}^{2} n_{e}^{2} \pi_{16} \frac{2 M_{y}}{\pi R^{4}} Z}\end{array}$ \\
\hline $\begin{array}{l}\delta(\Delta n)_{13}=\frac{n_{o}^{3}}{2} \pi_{16} \sigma_{12}-\frac{1}{2} \frac{\left(n_{o}^{3}+n_{e}^{3}\right) n_{o}^{2} n_{e}^{2} \pi_{45}^{2} \sigma_{23}^{2}}{n_{o}^{2}-n_{e}^{2}-n_{o}^{2} n_{e}^{2} \pi_{16} \sigma_{12}} \\
=n_{o}^{3} \pi_{16} \frac{M_{y}}{\pi R^{4}} Z-2 \frac{\left(n_{o}^{3}+n_{e}^{3}\right) n_{o}^{2} n_{e}^{2} \pi_{45}^{2} \frac{M_{y}^{2}}{\pi^{2} R^{8}} X^{2}}{n_{o}^{2}-n_{e}^{2}-n_{o}^{2} n_{e}^{2} \pi_{16} \frac{2 M_{y}}{\pi R^{4}} Z} \\
\simeq \frac{n_{o}^{3}}{2} \pi_{16} \sigma_{12}=n_{o}^{3} \pi_{16} \frac{M_{y}}{\pi R^{4}} Z\end{array}$ & $\begin{array}{l}\tan 2 \zeta_{Y}=\frac{2 n_{o}^{2} n_{e}^{2} \pi_{45} \sigma_{23}}{n_{o}^{2}-n_{e}^{2}-n_{o}^{2} n_{e}^{2} \pi_{16} \sigma_{12}} \\
=\frac{4 n_{o}^{2} n_{e}^{2} \pi_{45} \frac{M_{y}}{\pi R^{4}} X}{n_{o}^{2}-n_{e}^{2}-n_{o}^{2} n_{e}^{2} \pi_{16} \frac{2 M_{y}}{\pi R^{4}} Z}\end{array}$ \\
\hline $\begin{array}{l}\delta(\Delta n)_{12}=n_{o}^{3} \sigma_{12} \sqrt{\pi_{16}^{2}+\pi_{66}^{2}} \\
=n_{o}^{3} \frac{2 M_{y}}{\pi R^{4}} Z \sqrt{\pi_{16}^{2}+\pi_{66}^{2}} \\
\text { the birefringence is compensated on the optical } \\
\text { path length }\end{array}$ & $\tan 2 \zeta_{Z}=\frac{\pi_{66}}{\pi_{16}}$ \\
\hline $\begin{array}{l}\delta(\Delta n)_{23}=\frac{1}{2}\left(n_{o}^{3}+n_{e}^{3}\right) \frac{\left(\pi_{44} \sigma_{23}+\pi_{45} \sigma_{13}\right)^{2} n_{o}^{2} n_{e}^{2}}{n_{o}^{2}-n_{e}^{2}} \\
=2\left(n_{o}^{3}+n_{e}^{3}\right) \frac{\left(\pi_{44} \frac{M_{z}}{\pi R^{4}} X+\pi_{45} \frac{M_{z}}{\pi R^{4}} Y\right)^{2} n_{o}^{2} n_{e}^{2}}{n_{o}^{2}-n_{e}^{2}}\end{array}$ & $\begin{array}{l}\tan 2 \varsigma_{X}=\frac{2\left(\pi_{44} \sigma_{23}+\pi_{45} \sigma_{13}\right) n_{o}^{2} n_{e}^{2}}{n_{o}^{2}-n_{e}^{2}} \\
=4 \frac{\left(\pi_{44} \frac{M_{z}}{\pi R^{4}} X+\pi_{45} \frac{M_{z}}{\pi R^{4}} Y\right) n_{o}^{2} n_{e}^{2}}{n_{o}^{2}-n_{e}^{2}}\end{array}$ \\
\hline
\end{tabular}




\begin{tabular}{|l|c|c|}
\hline 1 & 2 & 3 \\
\hline$k \| Y$ & $n_{1}=n_{o}-\frac{1}{2} n_{o}^{3} \frac{\left(\pi_{44} \sigma_{13}-\pi_{45} \sigma_{23}\right)^{2} n_{o}^{2} n_{e}^{2}}{n_{o}^{2}-n_{e}^{2}}$ \\
$=$ & $n_{o}-2 n_{o}^{3} \frac{\left(\pi 4 \frac{M_{z}}{\pi R^{4}} Y-\pi_{45} \frac{M_{z}}{\pi R^{4}} X\right)^{2} n_{o}^{2} n_{e}^{2}}{n_{o}^{2}-n_{e}^{2}}$ \\
& $n_{3}=n_{e}+\frac{1}{2} n_{e}^{3} \frac{\left(\pi_{44} \sigma_{13}-\pi_{45} \sigma_{23}\right)^{2} n_{o}^{2} n_{e}^{2}}{n_{o}^{2}-n_{e}^{2}}$ \\
& $=n_{e}+2 n_{e}^{3} \frac{\left(\pi 4 \frac{M_{z}}{\pi R^{4}} Y-\pi_{45} \frac{M_{z}}{\pi R^{4}} X\right)^{2} n_{o}^{2} n_{e}^{2}}{n_{o}^{2}-n_{e}^{2}}$ \\
\cline { 2 - 3 } & not changed
\end{tabular}

Table 5. Changes in the optical indicatrix parameters under the torsion moment applied in crystals of the point symmetry groups $32,3 m$ and $\overline{3} m$.

\begin{tabular}{|c|c|c|}
\hline $\begin{array}{c}\text { Torsion } \\
\text { moment and } \\
\text { stress } \\
\text { components }\end{array}$ & $\begin{array}{l}\text { Direction of } \\
\text { light } \\
\text { propagation }\end{array}$ & Refractive indices \\
\hline 1 & 2 & 3 \\
\hline \multirow{3}{*}{$\begin{array}{l}M_{x}, \\
\sigma_{12}, \sigma_{13}\end{array}$} & $k \| X$ & not changed \\
\hline & $k \| Y$ & $\begin{array}{l}n_{1}=n_{o}+\frac{n_{o}^{3}}{2} \frac{n_{o}^{2} n_{e}^{2}\left(\pi_{44} \sigma_{13}+2 \pi_{41} \sigma_{12}\right)^{2}}{n_{o}^{2}-n_{e}^{2}} \\
=n_{o}+2 n_{o}^{3} \frac{n_{o}^{2} n_{e}^{2}\left(\pi_{44} \frac{M_{x}}{\pi R^{4}} Y+2 \pi_{41} \frac{M_{x}}{\pi R^{4}} Z\right)^{2}}{n_{o}^{2}-n_{e}^{2}} \\
n_{3}=n_{e}-\frac{n_{e}^{3}}{2} \frac{n_{o}^{2} n_{e}^{2}\left(\pi_{44} \sigma_{13}+2 \pi_{41} \sigma_{12}\right)^{2}}{n_{o}^{2}-n_{e}^{2}} \\
=n_{e}-2 n_{e}^{3} \frac{n_{o}^{2} n_{e}^{2}\left(\pi_{44} \frac{M_{x}}{\pi R^{4}} Y+2 \pi_{41} \frac{M_{x}}{\pi R^{4}} Z\right)^{2}}{n_{o}^{2}-n_{e}^{2}}\end{array}$ \\
\hline & $k \| Z$ & $\begin{array}{l}n_{1}=n_{o}-\frac{n_{o}^{3}}{2}\left(\pi_{14} \sigma_{13}+\pi_{66} \sigma_{12}\right)=n_{o}-n_{o}^{3} \frac{M_{x}}{\pi R^{4}}\left(\pi_{14} Y+\pi_{66} Z\right) \\
n_{2}=n_{o}+\frac{n_{o}^{3}}{2}\left(\pi_{14} \sigma_{13}+\pi_{66} \sigma_{12}\right)=n_{o}+n_{o}^{3} \frac{M_{x}}{\pi R^{4}}\left(\pi_{14} Y+\pi_{66} Z\right)\end{array}$ \\
\hline
\end{tabular}




\begin{tabular}{|l|l|}
\hline$\delta(\Delta n)_{13}=\frac{1}{2}\left(n_{o}^{3}+n_{e}^{3}\right) \frac{\left(\pi_{44} \sigma_{13}-\pi_{45} \sigma_{23}\right)^{2} n_{o}^{2} n_{e}^{2}}{n_{o}^{2}-n_{e}^{2}}$ & $\tan 2 \varsigma_{Y}=\frac{2\left(\pi_{44} \sigma_{13}-\pi_{45} \sigma_{23}\right) n_{o}^{2} n_{e}^{2}}{n_{o}^{2}-n_{e}^{2}}$ \\
$=2\left(n_{o}^{3}+n_{e}^{3}\right) \frac{\left(\pi_{44} \frac{M_{z}}{\pi R^{4}} Y-\pi_{45} \frac{M_{z}}{\pi R^{4}} X\right)^{2} n_{o}^{2} n_{e}^{2}}{n_{o}^{2}-n_{e}^{2}}$ & $=4 \frac{\left(\pi_{44} \frac{M_{z}}{\left.\pi R^{4} Y-\pi_{45} \frac{M_{z}}{\pi R^{4}} X\right) n_{o}^{2} n_{e}^{2}}\right.}{n_{o}^{2}-n_{e}^{2}}$ \\
\hline$\delta(\Delta n)_{12}=0$ & \\
\hline
\end{tabular}

\begin{tabular}{|l|l|}
\hline Induced birefringence & Angle of optical indicatrix rotation \\
\hline \hline$\delta(\Delta n)_{23}=0$ & $\tan 2 \zeta_{X}=0$ \\
\hline$\delta(\Delta n)_{13}=\frac{1}{2}\left(n_{o}^{3}+n_{e}^{3}\right) \frac{n_{o}^{2} n_{e}^{2}\left(\pi_{44} \sigma_{13}+2 \pi_{41} \sigma_{12}\right)^{2}}{n_{o}^{2}-n_{e}^{2}}$ & $\tan 2 \zeta_{Y}=\frac{2 n_{o}^{2} n_{e}^{2}\left(\pi_{44} \sigma_{13}+2 \pi_{41} \sigma_{12}\right)}{n_{o}^{2}-n_{e}^{2}}$ \\
$=2\left(n_{o}^{3}+n_{e}^{3}\right) \frac{n_{o}^{2} n_{e}^{2}\left(\pi_{44} \frac{M_{x}}{\pi R^{4}} Y+2 \pi_{41} \frac{M_{x}}{\pi R^{4}} Z\right)^{2}}{n_{o}^{2}-n_{e}^{2}}$ & $=\frac{4 n_{o}^{2} n_{e}^{2}\left(\pi_{44} \frac{M_{x}}{\pi R^{4}} Y+2 \pi_{41} \frac{M_{x}}{\pi R^{4}} Z\right)}{n_{o}^{2}-n_{e}^{2}}$ \\
\hline$\delta(\Delta n)_{12}=n_{o}^{3}\left(\pi_{14} \sigma_{13}+\pi_{66} \sigma_{12}\right)$ & \\
$=2 n_{o}^{3}\left(\pi_{14} \frac{M_{x}}{\pi R^{4}} Y+\pi_{66} \frac{M_{x}}{\pi R^{4}} Z\right)$ & $\tan 2 \zeta_{Z}= \pm \infty$ \\
\hline
\end{tabular}




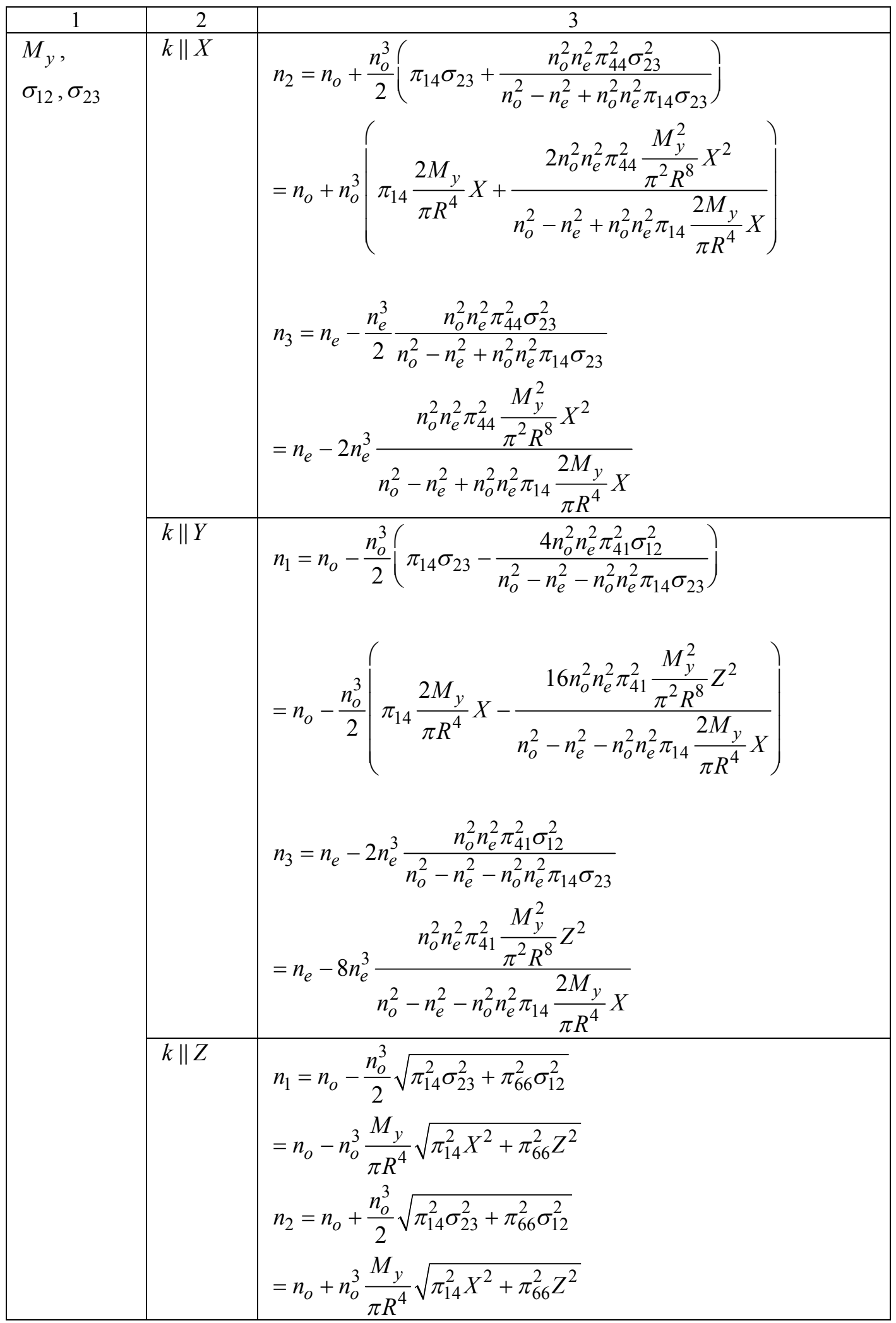




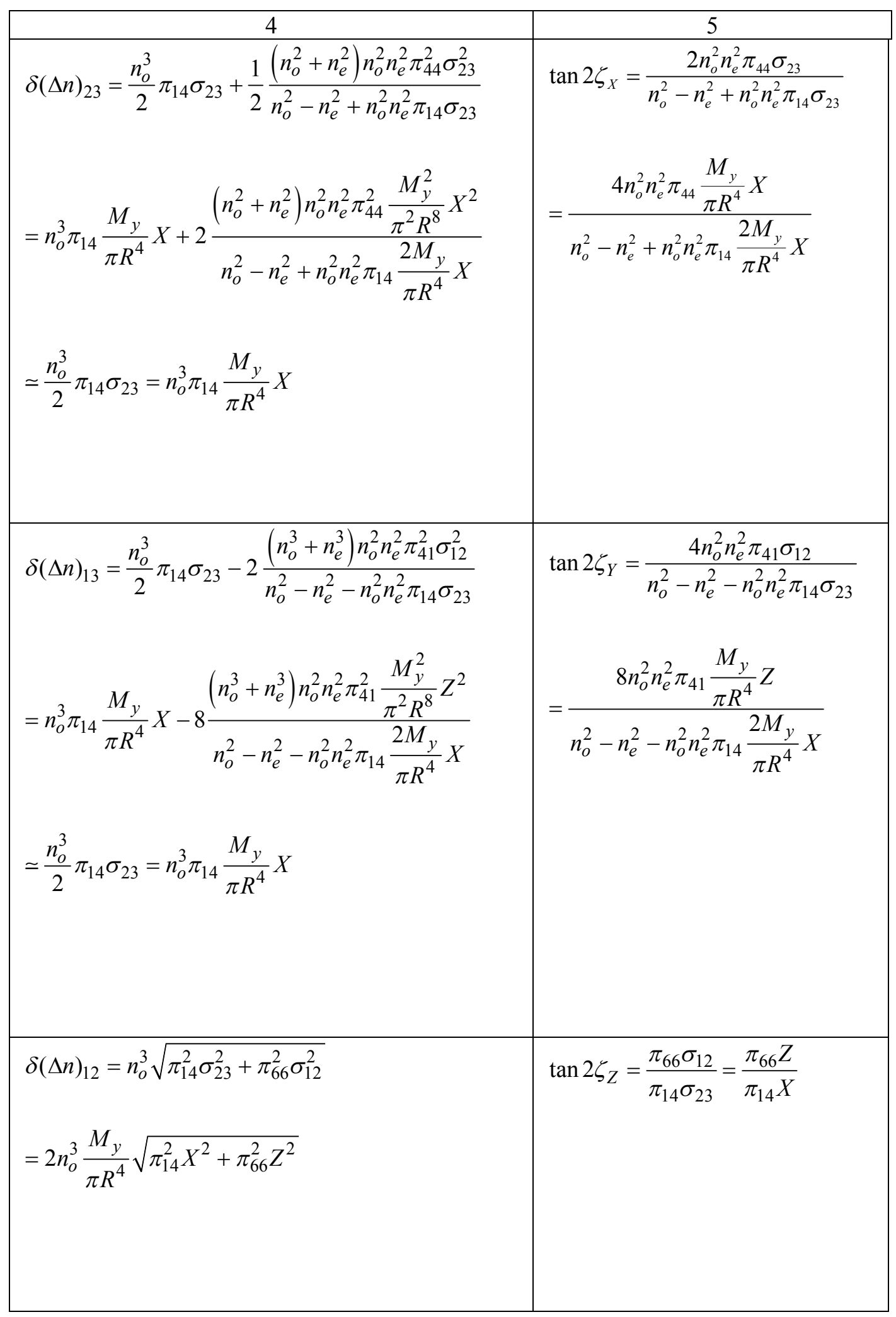


Skab I. et al

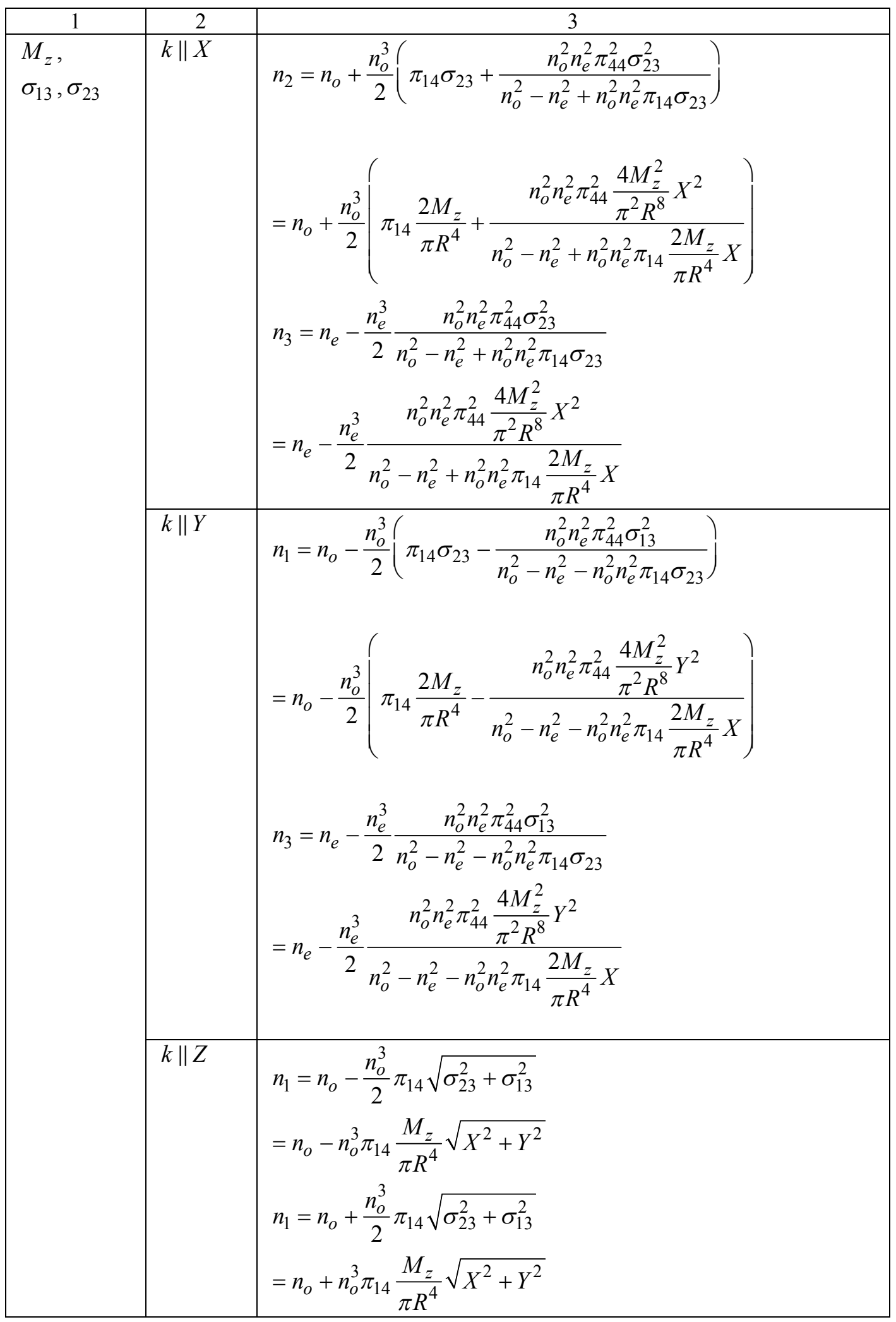

212

Ukr. J. Phys. Opt. 2010, V11, №4 


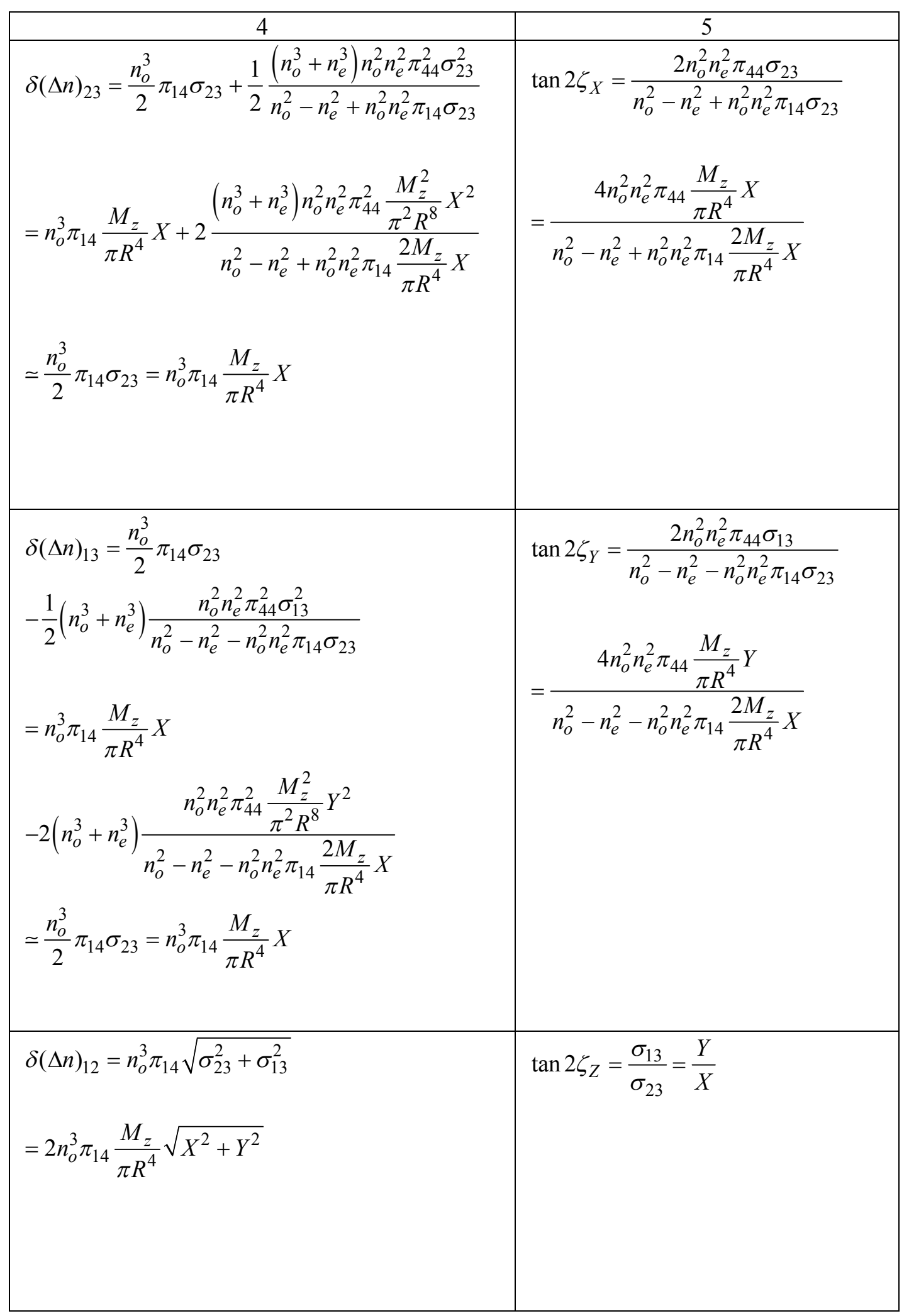


Table 6. Changes in the optical indicatrix parameters under the torsion moment applied in crystals of the point symmetry groups 3 and $\overline{3}$

\begin{tabular}{|c|c|c|}
\hline $\begin{array}{c}\text { Torsion } \\
\text { moment } \\
\text { and stress } \\
\text { components }\end{array}$ & $\begin{array}{l}\text { Direction of } \\
\text { light } \\
\text { propagation }\end{array}$ & Refractive indices \\
\hline 1 & 2 & 3 \\
\hline $\begin{array}{l}M_{x}, \\
\sigma_{12}, \sigma_{13}\end{array}$ & $k \| X$ & $\begin{array}{l}n_{2}=n_{o}-\frac{n_{o}^{3}}{2} \times \\
\times\left(\pi_{25} \sigma_{13}+2 \pi_{62} \sigma_{12}-\frac{n_{o}^{2} n_{e}^{2}\left(\pi_{45} \sigma_{13}+2 \pi_{52} \sigma_{12}\right)^{2}}{n_{o}^{2}-n_{e}^{2}-n_{o}^{2} n_{e}^{2}\left(\pi_{25} \sigma_{13}+2 \pi_{62} \sigma_{12}\right)}\right) \\
=n_{o}-n_{o}^{3} \frac{M_{x}}{\pi R^{4}} \times \\
\times\left(\pi_{25} Y+2 \pi_{62} Z-\frac{n_{o}^{2} n_{e}^{2} \frac{2 M_{x}}{\pi R^{4}}\left(\pi_{45} Y+2 \pi_{52} Z\right)^{2}}{n_{o}^{2}-n_{e}^{2}-n_{o}^{2} n_{e}^{2} \frac{2 M_{x}}{\pi R^{4}}\left(\pi_{25} Y+2 \pi_{62} Z\right)}\right) \\
n_{3}=n_{e}-\frac{n_{e}^{3}}{2} \frac{n_{o}^{2} n_{e}^{2}\left(\pi_{45} \sigma_{13}+2 \pi_{52} \sigma_{12}\right)^{2}}{n_{o}^{2}-n_{e}^{2}-n_{o}^{2} n_{e}^{2}\left(\pi_{25} \sigma_{13}+2 \pi_{62} \sigma_{12}\right)} \\
=n_{e}-2 n_{e}^{3} \frac{n_{o}^{2} n_{e}^{2} \frac{M_{x}^{2}}{\pi^{2} R^{8}}\left(\pi_{45} Y+2 \pi_{52} Z\right)^{2}}{n_{o}^{2}-n_{e}^{2}-n_{o}^{2} n_{e}^{2} \frac{2 M_{x}}{\pi R^{4}}\left(\pi_{25} Y+2 \pi_{62} Z\right)}\end{array}$ \\
\hline & $k \| Y$ & $\begin{array}{l}n_{1}=n_{o}-\frac{n_{o}^{3}}{2} \times \\
\times\left(\pi_{25} \sigma_{13}+2 \pi_{62} \sigma_{12}-\frac{n_{o}^{2} n_{e}^{2}\left(\pi_{44} \sigma_{13}+2 \pi_{41} \sigma_{12}\right)^{2}}{n_{o}^{2}-n_{e}^{2}-n_{o}^{2} n_{e}^{2}\left(\pi_{25} \sigma_{13}+2 \pi_{62} \sigma_{12}\right)}\right) \\
=n_{o}-n_{o}^{3} \frac{M_{x}}{\pi R^{4}} \times \\
\times\left(\pi_{25} Y+2 \pi_{62} Z-\frac{n_{o}^{2} n_{e}^{2} \frac{2 M_{x}}{\pi R^{4}}\left(\pi_{44} Y+2 \pi_{41} Z\right)^{2}}{n_{o}^{2}-n_{e}^{2}-n_{o}^{2} n_{e}^{2} \frac{2 M_{x}}{\pi R^{4}}\left(\pi_{25} Y+2 \pi_{62} Z\right)}\right) \\
n_{3}=n_{e}-\frac{n_{e}^{3}}{2} \frac{n_{o}^{2} n_{e}^{2}\left(\pi_{44} \sigma_{13}+2 \pi_{41} \sigma_{12}\right)^{2}}{n_{o}^{2}-n_{e}^{2}-n_{o}^{2} n_{e}^{2}\left(\pi_{25} \sigma_{13}+2 \pi_{62} \sigma_{12}\right)} \\
=n_{e}-2 n_{e}^{3} \frac{n_{o}^{2} n_{e}^{2} \frac{M_{x}^{2}}{\pi^{2} R^{8}}\left(\pi_{44} Y+2 \pi_{41} Z\right)^{2}}{n_{o}^{2}-n_{e}^{2}-n_{o}^{2} n_{e}^{2} \frac{2 M_{x}}{\pi R^{4}}\left(\pi_{25} Y+2 \pi_{62} Z\right)}\end{array}$ \\
\hline
\end{tabular}




\begin{tabular}{|c|c|}
\hline Induced birefringence & Angle of optical indicatrix rotation \\
\hline 4 & 5 \\
\hline $\begin{array}{l}\delta(\Delta n)_{23}=\frac{n_{o}^{3}}{2}\left(\pi_{25} \sigma_{13}+2 \pi_{62} \sigma_{12}\right) \\
-\frac{1}{2} \frac{\left(n_{o}^{3}+n_{e}^{3}\right) n_{o}^{2} n_{e}^{2}\left(\pi_{45} \sigma_{13}+2 \pi_{52} \sigma_{12}\right)^{2}}{n_{o}^{2}-n_{e}^{2}-n_{o}^{2} n_{e}^{2}\left(\pi_{25} \sigma_{13}+2 \pi_{62} \sigma_{12}\right)} \\
=n_{o}^{3} \frac{M_{x}}{\pi R^{4}}\left(\pi_{25} Y+2 \pi_{62} Z\right) \\
-2 \frac{M_{x}^{2}}{\pi^{2} R^{8}} \frac{\left(n_{o}^{3}+n_{e}^{3}\right) n_{o}^{2} n_{e}^{2}\left(\pi_{45} Y+2 \pi_{52} Z\right)^{2}}{n_{o}^{2}-n_{e}^{2}-n_{o}^{2} n_{e}^{2} \frac{2 M_{x}}{\pi R^{4}}\left(\pi_{25} Y+2 \pi_{62} Z\right)} \\
\simeq \frac{n_{o}^{3}}{2}\left(\pi_{25} \sigma_{13}+2 \pi_{62} \sigma_{12}\right)= \\
=-n_{o}^{3} \frac{M_{x}}{\pi R^{4}}\left(\pi_{25} Y+2 \pi_{62} Z\right)\end{array}$ & $\begin{array}{l}\tan 2 \zeta_{X}= \\
=\frac{2 n_{o}^{2} n_{e}^{2}\left(\pi_{45} \sigma_{13}+2 \pi_{52} \sigma_{12}\right)}{n_{o}^{2}-n_{e}^{2}-n_{o}^{2} n_{e}^{2}\left(\pi_{25} \sigma_{13}+2 \pi_{62} \sigma_{12}\right)} \\
=\frac{4 n_{o}^{2} n_{e}^{2} \frac{M_{x}}{\pi R^{4}}\left(\pi_{45} Y+2 \pi_{52} Z\right)}{n_{o}^{2}-n_{e}^{2}-n_{o}^{2} n_{e}^{2} \frac{2 M_{x}}{\pi R^{4}}\left(\pi_{25} Y+2 \pi_{62} Z\right)}\end{array}$ \\
\hline $\begin{array}{l}\delta(\Delta n)_{13}=\frac{n_{o}^{3}}{2}\left(\pi_{25} \sigma_{13}+2 \pi_{62} \sigma_{12}\right) \\
-\frac{1}{2} \frac{\left(n_{o}^{3}+n_{e}^{3}\right) n_{o}^{2} n_{e}^{2}\left(\pi_{44} \sigma_{13}+2 \pi_{41} \sigma_{12}\right)^{2}}{n_{o}^{2}-n_{e}^{2}-n_{o}^{2} n_{e}^{2}\left(\pi_{25} \sigma_{13}+2 \pi_{62} \sigma_{12}\right)} \\
=n_{o}^{3} \frac{M_{x}}{\pi R^{4}}\left(\pi_{25} Y+2 \pi_{62} Z\right) \\
-2 \frac{M_{x}^{2}}{\pi^{2} R^{8}} \frac{\left(n_{o}^{3}+n_{e}^{3}\right) n_{o}^{2} n_{e}^{2}\left(\pi_{44} Y+2 \pi_{41} Z\right)^{2}}{n_{o}^{2}-n_{e}^{2}-n_{o}^{2} n_{e}^{2} \frac{2 M_{x}}{\pi R^{4}}\left(\pi_{25} Y+2 \pi_{62} Z\right)} \\
\simeq \frac{n_{o}^{3}}{2}\left(\pi_{25} \sigma_{13}+2 \pi_{62} \sigma_{12}\right)= \\
=n_{o}^{3} \frac{M_{x}}{\pi R^{4}}\left(\pi_{25} Y+2 \pi_{62} Z\right)\end{array}$ & $\begin{array}{l}\tan 2 \zeta_{Y}= \\
=\frac{2 n_{o}^{2} n_{e}^{2}\left(\pi_{44} \sigma_{13}+2 \pi_{41} \sigma_{12}\right)}{n_{o}^{2}-n_{e}^{2}-n_{o}^{2} n_{e}^{2}\left(\pi_{25} \sigma_{13}+2 \pi_{62} \sigma_{12}\right)} \\
=\frac{4 n_{o}^{2} n_{e}^{2} \frac{M_{x}}{\pi R^{4}}\left(\pi_{44} Y+2 \pi_{41} Z\right)}{n_{o}^{2}-n_{e}^{2}-n_{o}^{2} n_{e}^{2} \frac{2 M_{x}}{\pi R^{4}}\left(\pi_{25} Y+2 \pi_{62} Z\right)}\end{array}$ \\
\hline
\end{tabular}




\begin{tabular}{|c|c|c|}
\hline 1 & 2 & 3 \\
\hline & $k \| Z$ & $\begin{array}{l}n_{1}=n_{o}-\frac{n_{o}^{3}}{2}\left(\left(\pi_{25}+\pi_{14}\right) \sigma_{13}+\left(\pi_{66}+2 \pi_{62}\right) \sigma_{12}\right) \\
=n_{o}-n_{o}^{3} \frac{M_{x}}{\pi R^{4}}\left(\left(\pi_{25}+\pi_{14}\right) Y+\left(\pi_{66}+2 \pi_{62}\right) Z\right) \\
n_{2}=n_{o}-\frac{n_{o}^{3}}{2}\left(\left(\pi_{25}-\pi_{14}\right) \sigma_{13}-\left(\pi_{66}-2 \pi_{62}\right) \sigma_{12}\right) \\
=n_{o}-n_{o}^{3} \frac{M_{x}}{\pi R^{4}}\left(\left(\pi_{25}-\pi_{14}\right) Y-\left(\pi_{66}-2 \pi_{62}\right) Z\right)\end{array}$ \\
\hline $\begin{array}{l}M_{y}, \\
\sigma_{12} \\
\sigma_{23}\end{array}$ & $k \| X$ & $\begin{array}{l}n_{2}=n_{o}-\frac{n_{o}^{3}}{2}\left(2 \pi_{62} \sigma_{12}-\pi_{14} \sigma_{23}-\frac{n_{o}^{2} n_{e}^{2}\left(\pi_{44} \sigma_{23}+2 \pi_{52} \sigma_{12}\right)^{2}}{n_{o}^{2}-n_{e}^{2}+n_{o}^{2} n_{e}^{2}\left(\pi_{14} \sigma_{23}-2 \pi_{62} \sigma_{12}\right)}\right) \\
=n_{o}-n_{o}^{3} \frac{M_{y}}{\pi R^{4}}\left(2 \pi_{62} Z-\pi_{14} X-\frac{n_{o}^{2} n_{e}^{2} \frac{2 M_{y}}{\pi R^{4}}\left(\pi_{44} X+2 \pi_{52} Z\right)^{2}}{\left.n_{o}^{2}-n_{e}^{2}+n_{o}^{2} n_{e}^{2} \frac{2 M_{y}}{\pi R^{4}}\left(\pi_{14} X-2 \pi_{62} Z\right)\right)}\right. \\
n_{3}=n_{e}-\frac{n_{e}^{3}}{2} \frac{n_{o}^{2} n_{e}^{2}\left(\pi_{44} \sigma_{23}+2 \pi_{52} \sigma_{12}\right)^{2}}{n_{o}^{2}-n_{e}^{2}+n_{o}^{2} n_{e}^{2}\left(\pi_{14} \sigma_{23}-2 \pi_{62} \sigma_{12}\right)} \\
=n_{e}-2 n_{e}^{3} \frac{n_{o}^{2} n_{e}^{2} \frac{M_{y}^{2}}{\pi^{2} R^{8}}\left(\pi_{44} X+2 \pi_{52} Z\right)^{2}}{n_{o}^{2}-n_{e}^{2}+n_{o}^{2} n_{e}^{2} \frac{2 M_{y}}{\pi R^{4}}\left(\pi_{14} X+2 \pi_{62} Z\right)}\end{array}$ \\
\hline & $k \| Y$ & 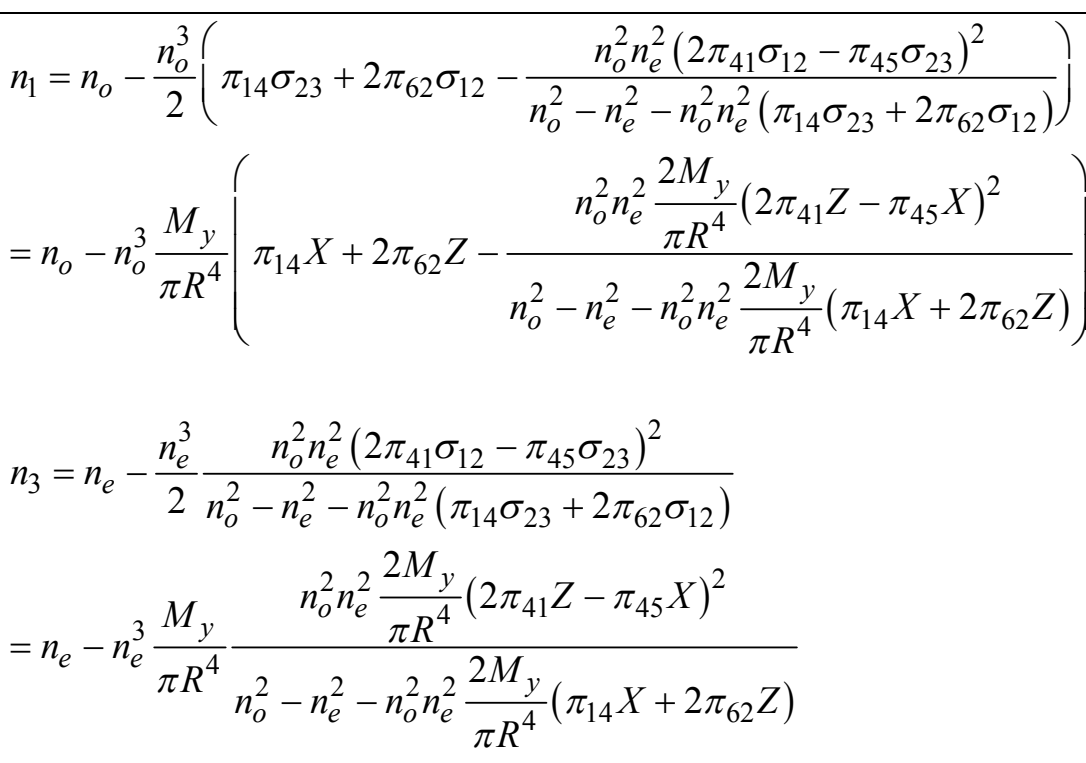 \\
\hline
\end{tabular}




\begin{tabular}{|c|c|}
\hline 4 & 5 \\
\hline $\begin{array}{l}\delta(\Delta n)_{12}=n_{o}^{3}\left(2 \pi_{62} \sigma_{12}-\pi_{14} \sigma_{13}\right) \\
=2 n_{o}^{3} \frac{M_{x}}{\pi R^{4}}\left(\pi_{62} Z-\pi_{14} Y\right)\end{array}$ & $\tan 2 \zeta_{Z}= \pm \infty$ \\
\hline $\begin{array}{l}\delta(\Delta n)_{23}=\frac{1}{2} n_{o}^{3}\left(2 \pi_{62} \sigma_{12}-\pi_{14} \sigma_{23}\right) \\
-\frac{1}{2} \frac{\left(n_{o}^{3}+n_{e}^{3}\right) n_{o}^{2} n_{e}^{2}\left(\pi_{44} \sigma_{23}+2 \pi_{52} \sigma_{12}\right)^{2}}{n_{o}^{2}-n_{e}^{2}+n_{o}^{2} n_{e}^{2}\left(\pi_{14} \sigma_{23}-2 \pi_{62} \sigma_{12}\right)} \\
=n_{o}^{3} \frac{M_{y}}{\pi R^{4}}\left(2 \pi_{62} Z-\pi_{14} X\right) \\
-2 \frac{\left(n_{o}^{3}+n_{e}^{3}\right) n_{o}^{2} n_{e}^{2} \frac{M_{y}^{2}}{\pi^{2} R^{8}}\left(\pi_{44} X+2 \pi_{52} Z\right)^{2}}{n_{o}^{2}-n_{e}^{2}+n_{o}^{2} n_{e}^{2} \frac{2 M_{y}}{\pi R^{4}}\left(\pi_{14} X-2 \pi_{62} Z\right)} \\
\simeq \frac{1}{2} n_{o}^{3}\left(2 \pi_{62} \sigma_{12}-\pi_{14} \sigma_{23}\right)= \\
=n_{o}^{3} \frac{M_{y}}{\pi R^{4}}\left(2 \pi_{62} Z-\pi_{14} X\right)\end{array}$ & $\begin{array}{l}\tan 2 \zeta_{X}= \\
=\frac{2 n_{o}^{2} n_{e}^{2}\left(\pi_{44} \sigma_{23}+2 \pi_{52} \sigma_{12}\right)}{n_{o}^{2}-n_{e}^{2}+n_{o}^{2} n_{e}^{2}\left(\pi_{14} \sigma_{23}-2 \pi_{62} \sigma_{12}\right)} \\
=\frac{4 n_{o}^{2} n_{e}^{2} \frac{M_{y}}{\pi R^{4}}\left(\pi_{44} X+2 \pi_{52} Z\right)}{n_{o}^{2}-n_{e}^{2}+n_{o}^{2} n_{e}^{2} \frac{2 M_{y}}{\pi R^{4}}\left(\pi_{14} X-2 \pi_{62} Z\right)}\end{array}$ \\
\hline $\begin{array}{l}\delta(\Delta n)_{23}=\frac{1}{2} n_{o}^{3}\left(\pi_{14} \sigma_{23}+2 \pi_{62} \sigma_{12}\right) \\
-\frac{1}{2} \frac{\left(n_{o}^{3}+n_{e}^{3}\right) n_{o}^{2} n_{e}^{2}\left(2 \pi_{41} \sigma_{12}-\pi_{45} \sigma_{23}\right)^{2}}{n_{o}^{2}-n_{e}^{2}-n_{o}^{2} n_{e}^{2}\left(\pi_{14} \sigma_{23}+2 \pi_{62} \sigma_{12}\right)} \\
=n_{o}^{3} \frac{M_{y}}{\pi R^{4}}\left(\pi_{14} X+2 \pi_{62} Z\right) \\
-2 \frac{\left(n_{o}^{3}+n_{e}^{3}\right) n_{o}^{2} n_{e}^{2} \frac{M_{y}^{2}}{\pi^{2} R^{8}}\left(2 \pi_{41} Z-\pi_{45} X\right)^{2}}{n_{o}^{2}-n_{e}^{2}-n_{o}^{2} n_{e}^{2} \frac{2 M_{y}}{\pi R^{4}}\left(\pi_{14} X-2 \pi_{62} Z\right)} \\
\simeq \frac{1}{2} n_{o}^{3}\left(\pi_{14} \sigma_{23}+2 \pi_{62} \sigma_{12}\right)= \\
=n_{o}^{3} \frac{M_{y}}{\pi R^{4}}\left(\pi_{14} X+2 \pi_{62} Z\right)\end{array}$ & $\begin{array}{l}\tan 2 \zeta_{Y}= \\
=\frac{2 n_{o}^{2} n_{e}^{2}\left(2 \pi_{41} \sigma_{12}-\pi_{45} \sigma_{23}\right)}{n_{o}^{2}-n_{e}^{2}-n_{o}^{2} n_{e}^{2}\left(\pi_{14} \sigma_{23}+2 \pi_{62} \sigma_{12}\right)} \\
=\frac{4 n_{o}^{2} n_{e}^{2} \frac{M_{y}}{\pi R^{4}}\left(2 \pi_{41} Z-\pi_{45} X\right)}{n_{o}^{2}-n_{e}^{2}-n_{o}^{2} n_{e}^{2} \frac{2 M_{y}}{\pi R^{4}}\left(\pi_{14} X+2 \pi_{62} Z\right)}\end{array}$ \\
\hline
\end{tabular}




\begin{tabular}{|c|c|c|}
\hline 1 & 2 & 3 \\
\hline & $k \| Z$ & $\begin{array}{l}n_{1}=n_{o}-\frac{n_{o}^{3}}{2}\left(2 \pi_{62} \sigma_{12}+\sqrt{\pi_{14}^{2} \sigma_{23}^{2}+\left(\pi_{25} \sigma_{23}+\pi_{66} \sigma_{12}\right)^{2}}\right) \\
=n_{o}-n_{o}^{3} \frac{M_{y}}{\pi R^{4}}\left(2 \pi_{62} Z+\sqrt{\pi_{14}^{2} X^{2}+\left(\pi_{25} X+\pi_{66} Z\right)^{2}}\right) \\
n_{2}=n_{o}-\frac{n_{o}^{3}}{2}\left(2 \pi_{62} \sigma_{12}-\sqrt{\pi_{14}^{2} \sigma_{23}^{2}+\left(\pi_{25} \sigma_{23}+\pi_{66} \sigma_{12}\right)^{2}}\right) \\
=n_{o}-n_{o}^{3} \frac{M_{y}}{\pi R^{4}}\left(2 \pi_{62} Z-\sqrt{\pi_{14}^{2} X^{2}+\left(\pi_{25} X+\pi_{66} Z\right)^{2}}\right)\end{array}$ \\
\hline $\begin{array}{l}M_{z} \\
\sigma_{13} \\
\sigma_{23}\end{array}$ & $k \| X$ & $\begin{array}{l}n_{2}=n_{o}-\frac{n_{o}^{3}}{2}\left(\pi_{25} \sigma_{13}-\pi_{14} \sigma_{23}-\frac{n_{o}^{2} n_{e}^{2}\left(\pi_{44} \sigma_{23}+\pi_{45} \sigma_{13}\right)^{2}}{n_{o}^{2}-n_{e}^{2}-n_{o}^{2} n_{e}^{2}\left(\pi_{25} \sigma_{13}-\pi_{14} \sigma_{23}\right)}\right) \\
=n_{o}-n_{o}^{3} \frac{M_{z}}{\pi R^{4}}\left(\pi_{25} Y-\pi_{14} X-\frac{n_{o}^{2} n_{e}^{2} \frac{2 M_{z}}{\pi R^{4}}\left(\pi_{44} X+\pi_{45} Y\right)^{2}}{n_{o}^{2}-n_{e}^{2}-n_{o}^{2} n_{e}^{2} \frac{2 M_{z}}{\pi R^{4}}\left(\pi_{25} Y-\pi_{14} X\right)}\right) \\
n_{3}=n_{e}-\frac{n_{e}^{3}}{2} \frac{n_{o}^{2} n_{e}^{2}\left(\pi_{44} \sigma_{23}+\pi_{45} \sigma_{13}\right)^{2}}{n_{o}^{2}-n_{e}^{2}-n_{o}^{2} n_{e}^{2}\left(\pi_{25} \sigma_{13}-\pi_{14} \sigma_{23}\right)} \\
=n_{e}-2 n_{e}^{3} \frac{n_{o}^{2} n_{e}^{2} \frac{M_{z}^{2}}{\pi^{2} R^{8}}\left(\pi_{44} X+\pi_{45} Y\right)^{2}}{n_{o}^{2}-n_{e}^{2}-n_{o}^{2} n_{e}^{2} \frac{2 M_{z}}{\pi R^{4}}\left(\pi_{25} Y-\pi_{14} X\right)}\end{array}$ \\
\hline & $k \| Y$ & $\begin{array}{l}n_{1}=n_{o}-\frac{n_{o}^{3}}{2}\left(\pi_{14} \sigma_{23}+\pi_{25} \sigma_{13}-\frac{n_{o}^{2} n_{e}^{2}\left(\pi_{44} \sigma_{13}-\pi_{45} \sigma_{23}\right)^{2}}{n_{o}^{2}-n_{e}^{2}-n_{o}^{2} n_{e}^{2}\left(\pi_{14} \sigma_{23}+\pi_{25} \sigma_{13}\right)}\right) \\
=n_{o}-n_{o}^{3} \frac{M_{z}}{\pi R^{4}}\left(\pi_{14} X+\pi_{25} Y-\frac{n_{o}^{2} n_{e}^{2} \frac{2 M_{z}}{\pi R^{4}}\left(\pi_{44} Y-\pi_{45} X\right)^{2}}{n_{o}^{2}-n_{e}^{2}-n_{o}^{2} n_{e}^{2} \frac{2 M_{z}}{\pi R^{4}}\left(\pi_{14} X+\pi_{25} Y\right)}\right. \\
n_{3}=n_{e}-\frac{n_{e}^{3}}{2} \frac{n_{o}^{2} n_{e}^{2}\left(\pi_{44} \sigma_{13}-\pi_{45} \sigma_{23}\right)^{2}}{n_{o}^{2}-n_{e}^{2}-n_{o}^{2} n_{e}^{2}\left(\pi_{14} \sigma_{23}+\pi_{25} \sigma_{13}\right)} \\
=n_{e}-2 n_{e}^{3} \frac{n_{o}^{2} n_{e}^{2} \frac{M_{z}^{2}}{\pi^{2} R^{8}}\left(\pi_{44} Y-\pi_{45} X\right)^{2}}{n_{o}^{2}-n_{e}^{2}-n_{o}^{2} n_{e}^{2} \frac{2 M_{z}}{\pi R^{4}}\left(\pi_{14} X+\pi_{25} Y\right)}\end{array}$ \\
\hline
\end{tabular}




\begin{tabular}{|c|c|}
\hline 4 & 5 \\
\hline $\begin{array}{l}\delta(\Delta n)_{12}=n_{o}^{3} \sqrt{\pi_{14}^{2} \sigma_{23}^{2}+\left(\pi_{25} \sigma_{23}+\pi_{66} \sigma_{12}\right)^{2}} \\
=2 n_{o}^{3} \frac{M_{y}}{\pi R^{4}} \sqrt{\pi_{14}^{2} X^{2}+\left(\pi_{25} X+\pi_{66} Z\right)^{2}}\end{array}$ & $\begin{array}{l}\tan 2 \zeta_{Z}=\frac{\pi_{25} \sigma_{23}+\pi_{66} \sigma_{12}}{\pi_{14} \sigma_{23}} \\
=\frac{\pi_{25} X+\pi_{66} Z}{\pi_{14} X}\end{array}$ \\
\hline $\begin{array}{l}\delta(\Delta n)_{23}=\frac{1}{2} n_{o}^{3}\left(\pi_{25} \sigma_{13}-\pi_{14} \sigma_{23}\right) \\
-\frac{1}{2} \frac{\left(n_{o}^{3}+n_{e}^{3}\right) n_{o}^{2} n_{e}^{2}\left(\pi_{44} \sigma_{23}+\pi_{45} \sigma_{13}\right)^{2}}{n_{o}^{2}-n_{e}^{2}-n_{o}^{2} n_{e}^{2}\left(\pi_{25} \sigma_{13}-\pi_{14} \sigma_{23}\right)} \\
=n_{o}^{3} \frac{M_{z}}{\pi R^{4}}\left(\pi_{25} Y-\pi_{14} X\right) \\
-2 \frac{\left(n_{o}^{3}+n_{e}^{3}\right) n_{o}^{2} n_{e}^{2} \frac{M_{z}^{2}}{\pi^{2} R^{8}}\left(\pi_{44} X+\pi_{45} Y\right)^{2}}{n_{o}^{2}-n_{e}^{2}-n_{o}^{2} n_{e}^{2} \frac{2 M_{z}}{\pi R^{4}}\left(\pi_{25} Y-\pi_{14} X\right)} \\
\simeq \frac{1}{2} n_{o}^{3}\left(\pi_{25} \sigma_{13}-\pi_{14} \sigma_{23}\right)= \\
=n_{o}^{3} \frac{M_{z}}{\pi R^{4}}\left(\pi_{25} Y-\pi_{14} X\right)\end{array}$ & $\begin{array}{l}\tan 2 \zeta_{X}=\frac{2 n_{o}^{2} n_{e}^{2}\left(\pi_{44} \sigma_{23}+\pi_{45} \sigma_{13}\right)}{n_{o}^{2}-n_{e}^{2}-n_{o}^{2} n_{e}^{2}\left(\pi_{25} \sigma_{13}-\pi_{14} \sigma_{23}\right)} \\
=\frac{4 n_{o}^{2} n_{e}^{2} \frac{M_{z}}{\pi R^{4}}\left(\pi_{44} X+\pi_{45} Y\right)}{n_{o}^{2}-n_{e}^{2}-n_{o}^{2} n_{e}^{2} \frac{2 M_{z}}{\pi R^{4}}\left(\pi_{25} Y-\pi_{14} X\right)}\end{array}$ \\
\hline $\begin{array}{l}\delta(\Delta n)_{13}=\frac{1}{2} n_{o}^{3}\left(\pi_{14} \sigma_{23}+\pi_{25} \sigma_{13}\right) \\
-\frac{1}{2} \frac{\left(n_{o}^{3}+n_{e}^{3}\right) n_{o}^{2} n_{e}^{2}\left(\pi_{44} \sigma_{13}-\pi_{45} \sigma_{23}\right)^{2}}{n_{o}^{2}-n_{e}^{2}-n_{o}^{2} n_{e}^{2}\left(\pi_{14} \sigma_{23}+\pi_{25} \sigma_{13}\right)} \\
=n_{o}^{3} \frac{M_{z}}{\pi R^{4}}\left(\pi_{14} X+\pi_{25} Y\right) \\
-2 \frac{\left(n_{o}^{3}+n_{e}^{3}\right) n_{o}^{2} n_{e}^{2} \frac{M_{z}^{2}}{\pi^{2} R^{8}}\left(\pi_{44} Y-\pi_{45} X\right)^{2}}{n_{o}^{2}-n_{e}^{2}-n_{o}^{2} n_{e}^{2} \frac{2 M_{z}}{\pi R^{4}}\left(\pi_{14} X+\pi_{25} Y\right)} \\
\simeq \frac{1}{2} n_{o}^{3}\left(\pi_{14} \sigma_{23}+\pi_{25} \sigma_{13}\right)= \\
=n_{o}^{3} \frac{M_{z}}{\pi R^{4}}\left(\pi_{14} X+\pi_{25} Y\right)\end{array}$ & $\begin{array}{l}\tan 2 \zeta_{Y}=\frac{2 n_{o}^{2} n_{e}^{2}\left(\pi_{44} \sigma_{13}-\pi_{45} \sigma_{23}\right)}{n_{o}^{2}-n_{e}^{2}-n_{o}^{2} n_{e}^{2}\left(\pi_{14} \sigma_{23}+\pi_{25} \sigma_{13}\right)} \\
=\frac{4 n_{o}^{2} n_{e}^{2} \frac{M_{z}}{\pi R^{4}}\left(\pi_{44} Y-\pi_{45} X\right)}{n_{o}^{2}-n_{e}^{2}-n_{o}^{2} n_{e}^{2} \frac{2 M_{z}}{\pi R^{4}}\left(\pi_{14} X+\pi_{25} Y\right)}\end{array}$ \\
\hline
\end{tabular}




\begin{tabular}{|l|c|c|}
\hline 1 & 2 & 3 \\
\hline$k \| Z$ & $n_{1}=n_{o}-\frac{n_{o}^{3}}{2}\left(\pi_{25} \sigma_{13}+\sqrt{\pi_{14}^{2} \sigma_{23}^{2}+\left(\pi_{25} \sigma_{23}+\pi_{14} \sigma_{13}\right)^{2}}\right)$ \\
& $=n_{o}-n_{o}^{3} \frac{M_{z}}{\pi R^{4}}\left(\pi_{25} Y+\sqrt{\pi_{14}^{2} X^{2}+\left(\pi_{25} X+\pi_{14} Y\right)^{2}}\right)$ \\
& $n_{2}=n_{o}-\frac{n_{o}^{3}}{2}\left(\pi_{25} \sigma_{13}-\sqrt{\pi_{14}^{2} \sigma_{23}^{2}+\left(\pi_{25} \sigma_{23}+\pi_{14} \sigma_{13}\right)^{2}}\right)$ \\
& $=n_{o}-n_{o}^{3} \frac{M_{z}}{\pi R^{4}}\left(\pi_{25} Y-\sqrt{\pi_{14}^{2} X^{2}+\left(\pi_{25} X+\pi_{14} Y\right)^{2}}\right)$ \\
\hline
\end{tabular}

Table 7. Changes in the optical indicatrix parameters under the torsion moment applied in crystals of the point symmetry groups $222, \mathrm{~mm} 2$ and $\mathrm{mmm}$.

\begin{tabular}{|c|c|c|}
\hline $\begin{array}{c}\text { Torsion } \\
\text { moment } \\
\text { and stress } \\
\text { components }\end{array}$ & $\begin{array}{l}\text { Direction of } \\
\text { light } \\
\text { propagation }\end{array}$ & Refractive indices \\
\hline 1 & 2 & 3 \\
\hline \multirow{3}{*}{$\begin{array}{l}M_{x}, \\
\sigma_{12}, \sigma_{13}\end{array}$} & $k \| X$ & not changed \\
\hline & $k \| Y$ & $\begin{array}{l}n_{1}^{\prime}=n_{1}+\frac{1}{2} \frac{n_{1}^{5} n_{3}^{2} \pi_{55}^{2} \sigma_{13}^{2}}{n_{1}^{2}-n_{3}^{2}}=n_{1}+\frac{n_{1}^{5} n_{3}^{2} \pi_{55}^{2}}{n_{1}^{2}-n_{3}^{2}} \frac{2 M_{x}^{2}}{\pi^{2} R^{8}} Y^{2} \\
n_{3}^{\prime}=n_{3}-\frac{1}{2} \frac{n_{1}^{2} n_{3}^{5} \pi_{55}^{2} \sigma_{13}^{2}}{n_{1}^{2}-n_{3}^{2}}=n_{3}-\frac{n_{1}^{2} n_{3}^{5} \pi_{55}^{2}}{n_{1}^{2}-n_{3}^{2}} \frac{2 M_{x}^{2}}{\pi^{2} R^{8}} Y^{2}\end{array}$ \\
\hline & $k \| Z$ & $\begin{array}{l}n_{1}^{\prime}=n_{1}+\frac{1}{2} \frac{n_{1}^{5} n_{2}^{2} \pi_{66}^{2} \sigma_{12}^{2}}{n_{1}^{2}-n_{2}^{2}}=n_{1}+\frac{n_{1}^{5} n_{2}^{2} \pi_{66}^{2}}{n_{1}^{2}-n_{2}^{2}} \frac{2 M_{x}^{2}}{\pi^{2} R^{8}} Z^{2} \\
n_{2}^{\prime}=n_{2}-\frac{1}{2} \frac{n_{1}^{2} n_{2}^{5} \pi_{66}^{2} \sigma_{12}^{2}}{n_{1}^{2}-n_{2}^{2}}=n_{2}-\frac{n_{1}^{2} n_{2}^{5} \pi_{66}^{2}}{n_{1}^{2}-n_{2}^{2}} \frac{2 M_{x}^{2}}{\pi^{2} R^{8}} Z^{2}\end{array}$ \\
\hline \multirow[t]{3}{*}{$\begin{array}{l}M_{y}, \\
\sigma_{12}, \sigma_{23}\end{array}$} & $k \| X$ & $\begin{array}{l}n_{2}^{\prime}=n_{2}+\frac{1}{2} \frac{n_{2}^{5} n_{3}^{2} \pi_{44}^{2} \sigma_{23}^{2}}{n_{2}^{2}-n_{3}^{2}}=n_{2}+\frac{n_{2}^{5} n_{3}^{2} \pi_{44}^{2}}{n_{2}^{2}-n_{3}^{2}} \frac{2 M_{y}^{2}}{\pi^{2} R^{8}} X^{2} \\
n_{3}^{\prime}=n_{3}-\frac{1}{2} \frac{n_{2}^{2} n_{3}^{5} \pi_{44}^{2} \sigma_{23}^{2}}{n_{2}^{2}-n_{3}^{2}}=n_{3}-\frac{n_{2}^{2} n_{3}^{5} \pi_{44}^{2}}{n_{2}^{2}-n_{3}^{2}} \frac{2 M_{y}^{2}}{\pi^{2} R^{8}} X^{2}\end{array}$ \\
\hline & $k \| Y$ & not changed \\
\hline & $k \| Z$ & $\begin{array}{l}n_{1}^{\prime}=n_{1}+\frac{1}{2} \frac{n_{1}^{5} n_{2}^{2} \pi_{66}^{2} \sigma_{12}^{2}}{n_{1}^{2}-n_{2}^{2}}=n_{1}+\frac{n_{1}^{5} n_{2}^{2} \pi_{66}^{2}}{n_{1}^{2}-n_{2}^{2}} \frac{2 M_{y}^{2}}{\pi^{2} R^{8}} Z^{2} \\
n_{2}^{\prime}=n_{2}-\frac{1}{2} \frac{n_{1}^{2} n_{2}^{5} \pi_{66}^{2} \sigma_{12}^{2}}{n_{1}^{2}-n_{2}^{2}}=n_{2}-\frac{n_{1}^{2} n_{2}^{5} \pi_{66}^{2}}{n_{1}^{2}-n_{2}^{2}} \frac{2 M_{y}^{2}}{\pi^{2} R^{8}} Z^{2}\end{array}$ \\
\hline
\end{tabular}




\begin{tabular}{|l|l|}
\hline \multicolumn{1}{|c|}{4} & \multicolumn{1}{|c|}{5} \\
\hline$\delta(\Delta n)_{12}=n_{o}^{3} \sqrt{\pi_{14}^{2} \sigma_{23}^{2}+\left(\pi_{25} \sigma_{23}+\pi_{14} \sigma_{13}\right)^{2}}$ & $\tan 2 \zeta_{Z}=\frac{\pi_{25} \sigma_{23}+\pi_{14} \sigma_{13}}{\pi_{14} \sigma_{23}}$ \\
$=2 n_{o}^{3} \frac{M_{z}}{\pi R^{4}} \sqrt{\pi_{14}^{2} X^{2}+\left(\pi_{25} X+\pi_{14} Y\right)^{2}}$ & $=\frac{\pi_{25} X+\pi_{14} Y}{\pi_{14} X}$ \\
& \\
& \\
\hline
\end{tabular}

\begin{tabular}{|c|c|}
\hline Induced birefringence & Angle of optical indicatrix rotation \\
\hline 4 & 5 \\
\hline$\delta(\Delta n)_{23}=0$ & $\tan 2 \zeta_{X}=0$ \\
\hline $\begin{array}{l}\delta(\Delta n)_{13}=\frac{1}{2} \frac{n_{1}^{2} n_{3}^{2}\left(n_{1}^{3}+n_{3}^{3}\right)}{n_{1}^{2}-n_{3}^{2}} \pi_{55}^{2} \sigma_{13}^{2} \\
=\frac{2 n_{1}^{2} n_{3}^{2}\left(n_{1}^{3}+n_{3}^{3}\right)}{n_{1}^{2}-n_{3}^{2}} \pi_{55}^{2} \frac{M_{x}^{2}}{\pi^{2} R^{8}} Y^{2}\end{array}$ & $\begin{array}{l}\tan 2 \zeta_{Y}=\frac{2 n_{1}^{2} n_{3}^{2} \pi_{55} \sigma_{13}}{n_{1}^{2}-n_{3}^{2}} \\
=\frac{4 n_{1}^{2} n_{3}^{2} \pi_{55}}{n_{1}^{2}-n_{3}^{2}} \frac{M_{x}}{\pi R^{4}} Y\end{array}$ \\
\hline $\begin{array}{l}\delta(\Delta n)_{12}=\frac{1}{2} \frac{n_{1}^{2} n_{2}^{2}\left(n_{1}^{3}+n_{2}^{3}\right)}{n_{1}^{2}-n_{2}^{2}} \pi_{66}^{2} \sigma_{12}^{2} \\
=\frac{2 n_{1}^{2} n_{2}^{2}\left(n_{1}^{3}+n_{2}^{3}\right)}{n_{1}^{2}-n_{2}^{2}} \pi_{66}^{2} \frac{M_{x}^{2}}{\pi^{2} R^{8}} Z^{2}\end{array}$ & $\begin{array}{l}\tan 2 \zeta_{Z}=\frac{2 n_{1}^{2} n_{2}^{2} \pi_{66} \sigma_{12}}{n_{1}^{2}-n_{2}^{2}} \\
=\frac{4 n_{1}^{2} n_{2}^{2} \pi_{66}}{n_{1}^{2}-n_{2}^{2}} \frac{M_{x}}{\pi R^{4}} Z\end{array}$ \\
\hline $\begin{array}{l}\delta(\Delta n)_{23}=\frac{1}{2} \frac{n_{2}^{2} n_{3}^{2}\left(n_{2}^{3}+n_{3}^{3}\right)}{n_{2}^{2}-n_{3}^{2}} \pi_{44}^{2} \sigma_{23}^{2} \\
=\frac{2 n_{2}^{2} n_{3}^{2}\left(n_{2}^{3}+n_{3}^{3}\right)}{n_{2}^{2}-n_{3}^{2}} \pi_{44}^{2} \frac{M_{y}^{2}}{\pi^{2} R^{8}} X^{2}\end{array}$ & $\begin{array}{l}\tan 2 \zeta_{X}=\frac{2 n_{3}^{2} n_{2}^{2} \pi_{44} \sigma_{23}}{n_{3}^{2}-n_{2}^{2}} \\
=\frac{4 n_{3}^{2} n_{2}^{2} \pi_{44}}{n_{3}^{2}-n_{2}^{2}} \frac{M_{y}}{\pi R^{4}} X\end{array}$ \\
\hline$\delta(\Delta n)_{13}=0$ & $\tan 2 \zeta_{Y}=0$ \\
\hline $\begin{array}{l}\delta(\Delta n)_{12}=\frac{1}{2} \frac{n_{1}^{2} n_{2}^{2}\left(n_{1}^{3}+n_{2}^{3}\right)}{n_{1}^{2}-n_{2}^{2}} \pi_{66}^{2} \sigma_{12}^{2} \\
=\frac{2 n_{1}^{2} n_{2}^{2}\left(n_{1}^{3}+n_{2}^{3}\right)}{n_{1}^{2}-n_{2}^{2}} \pi_{66}^{2} \frac{M_{y}^{2}}{\pi^{2} R^{8}} Z^{2}\end{array}$ & $\begin{array}{l}\tan 2 \zeta_{Z}=\frac{2 n_{1}^{2} n_{2}^{2} \pi_{66} \sigma_{12}}{n_{2}^{2}-n_{1}^{2}} \\
=\frac{4 n_{1}^{2} n_{2}^{2} \pi_{66}}{n_{2}^{2}-n_{1}^{2}} \frac{M_{y}}{\pi R^{4}} Z\end{array}$ \\
\hline
\end{tabular}




\begin{tabular}{|l|l|l|}
\hline 1 & \multicolumn{1}{|c|}{2} & 3 \\
\hline$M_{z}$, & $k \| X$ & $n_{2}^{\prime}=n_{2}+\frac{1}{2} \frac{n_{2}^{5} n_{3}^{2} \pi_{44}^{2} \sigma_{23}^{2}}{n_{2}^{2}-n_{3}^{2}}=n_{2}+\frac{n_{2}^{5} n_{3}^{2} \pi_{44}^{2}}{n_{2}^{2}-n_{3}^{2}} \frac{2 M_{z}^{2}}{\pi^{2} R^{8}} X^{2}$ \\
& & $n_{3}^{\prime}=n_{3}-\frac{1}{2} \frac{n_{2}^{2} n_{3}^{5} \pi_{44}^{2} \sigma_{23}^{2}}{n_{2}^{2}-n_{3}^{2}}=n_{3}-\frac{n_{2}^{2} n_{3}^{5} \pi_{44}^{2}}{n_{2}^{2}-n_{3}^{2}} \frac{2 M_{z}^{2}}{\pi^{2} R^{8}} X^{2}$ \\
\cline { 2 - 3 } & $k \| Y$ & $n_{1}^{\prime}=n_{1}+\frac{1}{2} \frac{n_{1}^{5} n_{3}^{2} \pi_{55}^{2} \sigma_{13}^{2}}{n_{1}^{2}-n_{3}^{2}}=n_{1}+\frac{n_{1}^{5} n_{3}^{2} \pi_{55}^{2}}{n_{1}^{2}-n_{3}^{2}} \frac{2 M_{z}^{2}}{\pi^{2} R^{8}} Y^{2}$ \\
& $n_{3}^{\prime}=n_{3}-\frac{1}{2} \frac{n_{1}^{2} n_{3}^{5} \pi_{55}^{2} \sigma_{13}^{2}}{n_{1}^{2}-n_{3}^{2}}=n_{3}-\frac{n_{1}^{2} n_{3}^{5} \pi_{55}^{2}}{n_{1}^{2}-n_{3}^{2}} \frac{2 M_{z}^{2}}{\pi^{2} R^{8}} Y^{2}$ \\
\cline { 2 - 3 } & $k \| Z$ & not changed \\
\hline
\end{tabular}

Table 8. Changes in the optical indicatrix parameters under the torsion moment applied in crystals of the point symmetry groups $2 / \mathrm{m}$, $\mathrm{m}$ and $2(2 \| Y, m \perp Y)$.

\begin{tabular}{|c|c|c|}
\hline $\begin{array}{c}\text { Torsion } \\
\text { moment } \\
\text { and stress } \\
\text { components }\end{array}$ & $\begin{array}{l}\text { Direction of } \\
\text { light } \\
\text { propagation }\end{array}$ & Refractive indices \\
\hline 1 & 2 & 3 \\
\hline $\begin{array}{l}M_{x} \\
\sigma_{12}, \sigma_{13}\end{array}$ & $k \| X$ & $\begin{array}{l}n_{2}^{\prime}=n_{2}-\frac{n_{2}^{3}}{2}\left(\pi_{25} \sigma_{13}+\frac{n_{2}^{2} n_{3}^{2} \pi_{46}^{2} \sigma_{12}^{2}}{n_{3}^{2}-n_{2}^{2}+\left(\pi_{25}-\pi_{35}\right) \sigma_{13} n_{2}^{2} n_{3}^{2}}\right) \\
=n_{2}-\frac{n_{2}^{3}}{2}\left(\pi_{25} \frac{2 M_{x}}{\pi R^{4}} Y+\frac{n_{2}^{2} n_{3}^{2} \pi_{46}^{2} \frac{4 M_{x}^{2}}{\pi^{2} R^{8}} Z^{2}}{\left.n_{3}^{2}-n_{2}^{2}+n_{2}^{2} n_{3}^{2}\left(\pi_{25}-\pi_{35}\right) \frac{2 M_{x}}{\pi R^{4}} Y\right)}\right. \\
\simeq n_{2}-\frac{n_{2}^{3}}{2} \pi_{25} \sigma_{13}=n_{2}-n_{2}^{3} \pi_{25} \frac{M_{x}}{\pi R^{4}} Y \\
n_{3}^{\prime}=n_{3}-\frac{n_{3}^{3}}{2}\left(\pi_{35} \sigma_{13}-\frac{n_{2}^{2} n_{3}^{2} \pi_{46}^{2} \sigma_{12}^{2}}{n_{3}^{2}-n_{2}^{2}+\left(\pi_{25}-\pi_{35}\right) \sigma_{13} n_{2}^{2} n_{3}^{2}}\right) \\
=n_{3}-\frac{n_{3}^{3}}{2}\left(\pi_{35} \frac{2 M_{x}}{\pi R^{4}} Y-\frac{n_{2}^{2} n_{3}^{2} \pi_{46}^{2} \frac{4 M_{x}^{2}}{\pi^{2} R^{8}} Z^{2}}{\left.n_{3}^{2}-n_{2}^{2}+n_{2}^{2} n_{3}^{2}\left(\pi_{25}-\pi_{35}\right) \frac{2 M_{x}}{\pi R^{4}} Y\right)}\right) \\
\simeq n_{3}-\frac{n_{3}^{3}}{2} \pi_{35} \sigma_{13}=n_{3}-n_{3}^{3} \pi_{35} \frac{M_{x}}{\pi R^{4}} Y\end{array}$ \\
\hline
\end{tabular}




\begin{tabular}{|c|c|}
\hline 4 & 5 \\
\hline $\begin{array}{l}\delta(\Delta n)_{23}=\frac{1}{2} \frac{n_{2}^{2} n_{3}^{2}\left(n_{2}^{3}+n_{3}^{3}\right)}{n_{2}^{2}-n_{3}^{2}} \pi_{44}^{2} \sigma_{23}^{2} \\
=\frac{2 n_{2}^{2} n_{3}^{2}\left(n_{2}^{3}+n_{3}^{3}\right)}{n_{2}^{2}-n_{3}^{2}} \pi_{44}^{2} \frac{M_{z}^{2}}{\pi^{2} R^{8}} X^{2}\end{array}$ & $\begin{array}{l}\tan 2 \zeta_{X}=\frac{2 n_{3}^{2} n_{2}^{2} \pi_{44} \sigma_{23}}{n_{3}^{2}-n_{2}^{2}} \\
=\frac{4 n_{3}^{2} n_{2}^{2} \pi_{44}}{n_{3}^{2}-n_{2}^{2}} \frac{M_{z}}{\pi R^{4}} X\end{array}$ \\
\hline $\begin{array}{l}\delta(\Delta n)_{13}=\frac{1}{2} \frac{n_{1}^{2} n_{3}^{2}\left(n_{1}^{3}+n_{3}^{3}\right)}{n_{1}^{2}-n_{3}^{2}} \pi_{55}^{2} \sigma_{13}^{2} \\
=\frac{2 n_{1}^{2} n_{3}^{2}\left(n_{1}^{3}+n_{3}^{3}\right)}{n_{1}^{2}-n_{3}^{2}} \pi_{55}^{2} \frac{M_{z}^{2}}{\pi^{2} R^{8}} Y^{2}\end{array}$ & $\begin{array}{l}\tan 2 \zeta_{Y}=\frac{2 n_{1}^{2} n_{3}^{2} \pi_{55} \sigma_{13}}{n_{1}^{2}-n_{3}^{2}} \\
=\frac{4 n_{1}^{2} n_{3}^{2} \pi_{55}}{n_{1}^{2}-n_{3}^{2}} \frac{M_{z}}{\pi R^{4}} Y\end{array}$ \\
\hline$\delta(\Delta n)_{12}=0$ & $\tan 2 \zeta_{Z}=0$ \\
\hline
\end{tabular}

\begin{tabular}{|c|c|}
\hline Induced birefringence & Angle of optical indicatrix rotation \\
\hline$\delta(\Delta n)_{23}=\frac{1}{2}\left(n_{3}^{3} \pi_{35}-n_{2}^{3} \pi_{25}\right) \sigma_{13}$ & $\tan 2 \zeta_{X}=\frac{5}{n_{3}^{2}-n_{2}^{2}+n_{2}^{2} n_{3}^{2}\left(\pi_{25}-\pi_{35}\right) \sigma_{13}}$ \\
$+\frac{1}{2} \frac{\left(n_{3}^{3}-n_{2}^{3}\right) n_{2}^{2} n_{3}^{2} \pi_{46}^{2} \sigma_{12}^{2}}{n_{3}^{2}-n_{2}^{2}+\left(\pi_{25}-\pi_{35}\right) \sigma_{13} n_{2}^{2} n_{3}^{2}}$ & $=\frac{4 n_{2}^{2} n_{3}^{2} \pi_{46} \frac{M_{x}}{\pi R^{4}} Z}{n_{3}^{2}-n_{2}^{2}+n_{2}^{2} n_{3}^{2}\left(\pi_{25}-\pi_{35}\right) \frac{2 M_{x}}{\pi R^{4}} Y}$ \\
$=\left(n_{3}^{3} \pi_{35}-n_{2}^{3} \pi_{25}\right) \frac{M_{x}}{\pi R^{4}} Y$ & \\
$+2 \frac{\left(n_{3}^{3}-n_{2}^{3}\right) n_{2}^{2} n_{3}^{2} \pi_{46}^{2} \frac{M_{x}^{2}}{\pi^{2} R^{8}} Z^{2}}{n_{3}^{2}-n_{2}^{2}+n_{2}^{2} n_{3}^{2}\left(\pi_{25}-\pi_{35}\right) \frac{2 M_{x}}{\pi R^{4}} Y}$ & \\
$\simeq \frac{1}{2}\left(n_{3}^{3} \pi_{35}-n_{2}^{3} \pi_{25}\right) \sigma_{13}$ & \\
$=\left(n_{3}^{3} \pi_{35}-n_{2}^{3} \pi_{25}\right) \frac{M_{x}}{\pi R^{4}} Y$ & \\
\hline
\end{tabular}




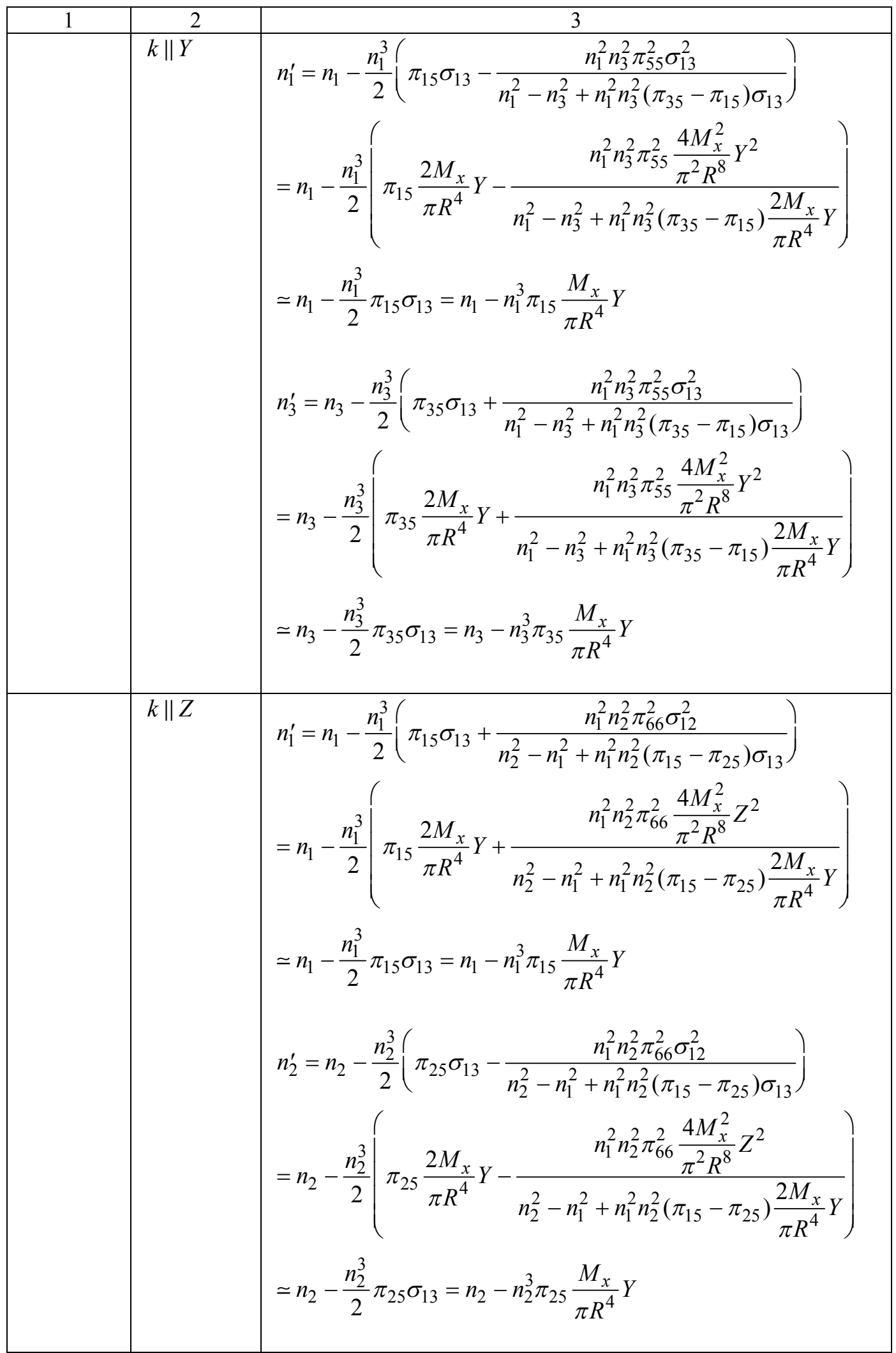




\begin{tabular}{|c|c|}
\hline 4 & 5 \\
\hline $\begin{array}{l}\delta(\Delta n)_{13}=\frac{1}{2}\left(n_{3}^{3} \pi_{35}-n_{1}^{3} \pi_{15}\right) \sigma_{13} \\
+\frac{1}{2} \frac{\left(n_{1}^{3}+n_{3}^{3}\right) n_{1}^{2} n_{3}^{2} \pi_{55}^{2} \sigma_{13}^{2}}{n_{1}^{2}-n_{3}^{2}+n_{1}^{2} n_{3}^{2}\left(\pi_{35}-\pi_{15}\right) \sigma_{13}} \\
=\left(n_{3}^{3} \pi_{35}-n_{1}^{3} \pi_{15}\right) \frac{M_{x}}{\pi R^{4}} Y \\
+2 \frac{\left(n_{1}^{3}+n_{3}^{3}\right) n_{1}^{2} n_{3}^{2} \pi_{55}^{2} \frac{M_{x}^{2}}{\pi^{2} R^{8}} Y^{2}}{n_{1}^{2}-n_{3}^{2}+n_{1}^{2} n_{3}^{2}\left(\pi_{35}-\pi_{15}\right) \frac{2 M_{x}}{\pi R^{4}} Y} \\
\simeq \frac{1}{2}\left(n_{3}^{3} \pi_{35}-n_{1}^{3} \pi_{15}\right) \sigma_{13} \\
=\left(n_{3}^{3} \pi_{35}-n_{1}^{3} \pi_{15}\right) \frac{M_{x}}{\pi R^{4}} Y\end{array}$ & $\begin{array}{l}\tan 2 \zeta_{Y}=\frac{2 n_{1}^{2} n_{3}^{2} \pi_{55} \sigma_{13}}{n_{1}^{2}-n_{3}^{2}+n_{1}^{2} n_{3}^{2}\left(\pi_{35}-\pi_{15}\right) \sigma_{13}} \\
=\frac{4 n_{1}^{2} n_{3}^{2} \pi_{55} \frac{M_{x}}{\pi R^{4}} Y}{n_{1}^{2}-n_{3}^{2}+n_{1}^{2} n_{3}^{2}\left(\pi_{35}-\pi_{15}\right) \frac{2 M_{x}}{\pi R^{4}} Y}\end{array}$ \\
\hline $\begin{array}{l}\delta(\Delta n)_{12}=\frac{1}{2}\left(n_{2}^{3} \pi_{25}-n_{1}^{3} \pi_{15}\right) \sigma_{13} \\
+\frac{1}{2} \frac{\left(n_{2}^{3}-n_{1}^{3}\right) n_{1}^{2} n_{2}^{2} \pi_{66}^{2} \sigma_{12}^{2}}{n_{2}^{2}-n_{1}^{2}+n_{1}^{2} n_{2}^{2}\left(\pi_{15}-\pi_{25}\right) \sigma_{13}} \\
=\left(n_{2}^{3} \pi_{25}-n_{1}^{3} \pi_{15}\right) \frac{M_{x}}{\pi R^{4}} Y \\
+2 \frac{\left(n_{2}^{3}-n_{1}^{3}\right) n_{1}^{2} n_{2}^{2} \pi_{66}^{2} \frac{M_{x}^{2}}{\pi^{2} R^{8}} Z^{2}}{n_{2}^{2}-n_{1}^{2}+n_{1}^{2} n_{2}^{2}\left(\pi_{15}-\pi_{25}\right) \frac{2 M_{x}}{\pi R^{4}} Y} \\
\simeq \frac{1}{2}\left(n_{2}^{3} \pi_{25}-n_{1}^{3} \pi_{15}\right) \sigma_{13} \\
=\left(n_{2}^{3} \pi_{25}-n_{1}^{3} \pi_{15}\right) \frac{M_{x}}{\pi R^{4}} Y\end{array}$ & $\begin{array}{l}\tan 2 \zeta_{Z}=\frac{2 n_{1}^{2} n_{2}^{2} \pi_{66} \sigma_{12}}{n_{2}^{2}-n_{1}^{2}+n_{1}^{2} n_{2}^{2}\left(\pi_{15}-\pi_{25}\right) \sigma_{13}} \\
=\frac{4 n_{1}^{2} n_{2}^{2} \pi_{66} \frac{M_{x}}{\pi R^{4}} Z}{n_{2}^{2}-n_{1}^{2}+n_{1}^{2} n_{2}^{2}\left(\pi_{15}-\pi_{25}\right) \frac{2 M_{x}}{\pi R^{4}} Y}\end{array}$ \\
\hline
\end{tabular}




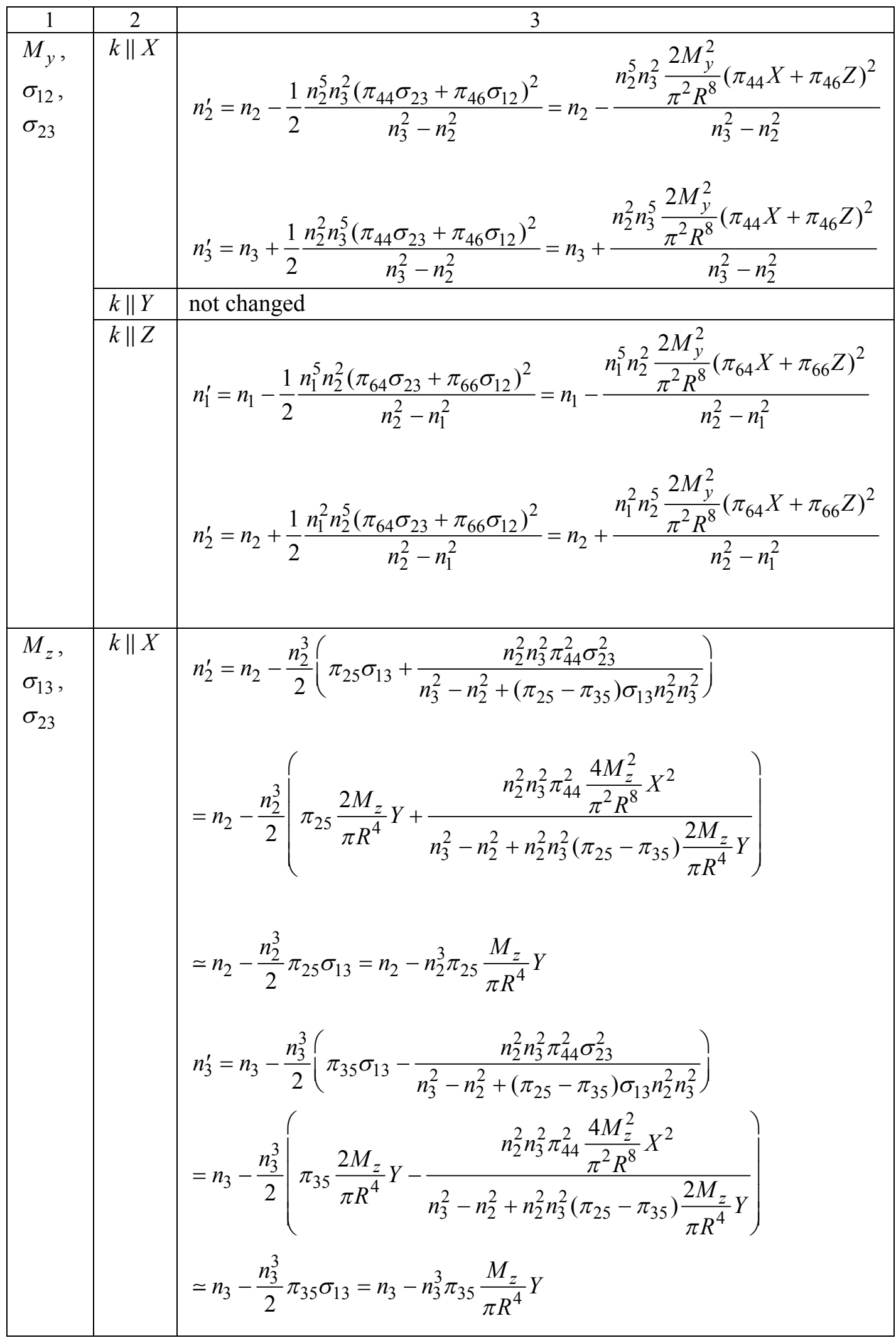




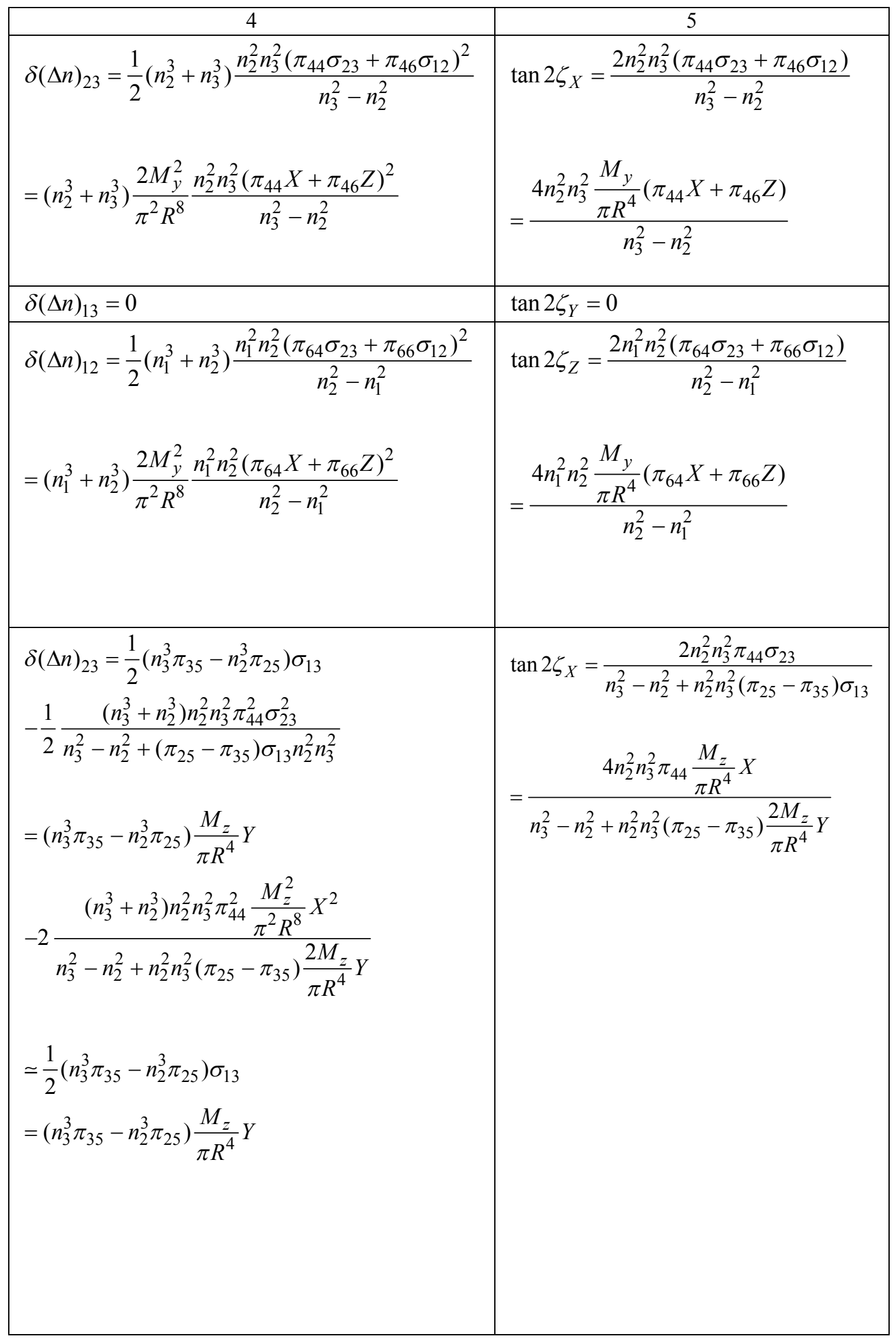




\begin{tabular}{|c|c|c|}
\hline 1 & 2 & 3 \\
\hline & $k \| Y$ & 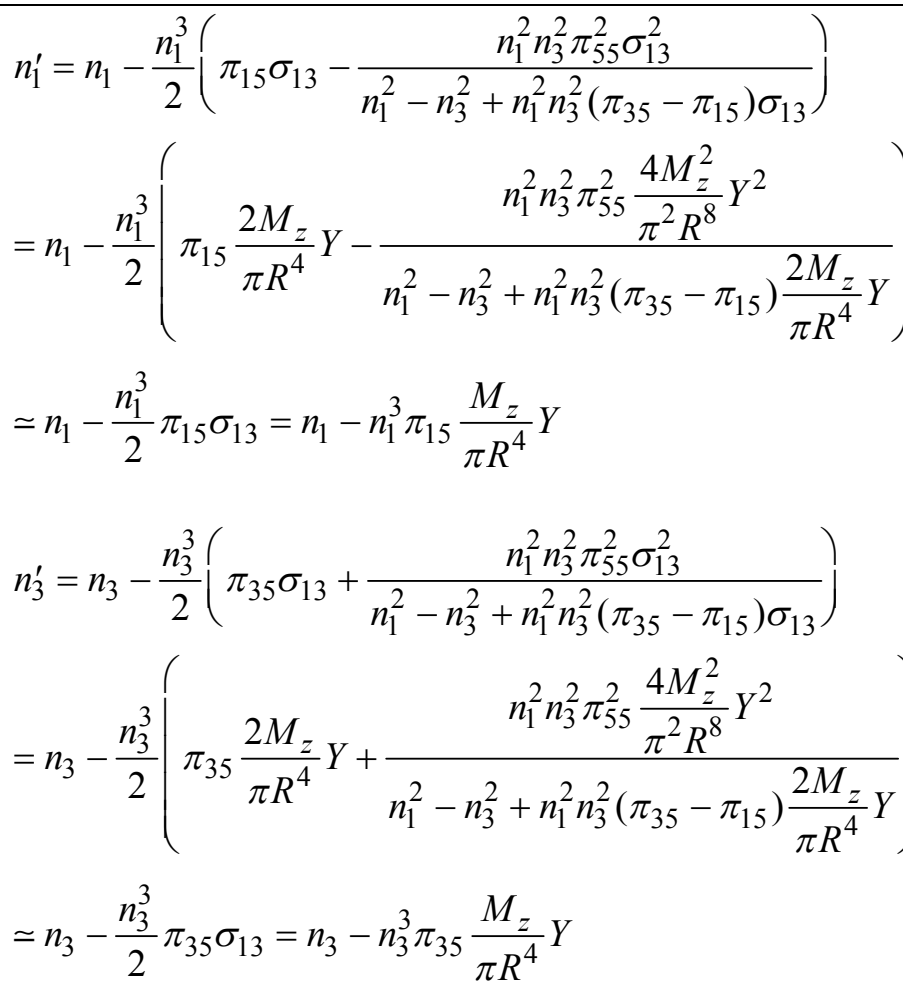 \\
\hline & $k \| Z$ & $\begin{array}{l}n_{1}^{\prime}=n_{1}-\frac{n_{1}^{3}}{2}\left(\pi_{15} \sigma_{13}+\frac{n_{1}^{2} n_{2}^{2} \pi_{64}^{2} \sigma_{23}^{2}}{n_{2}^{2}-n_{1}^{2}+n_{1}^{2} n_{2}^{2}\left(\pi_{15}-\pi_{25}\right) \sigma_{13}}\right) \\
=n_{1}-\frac{n_{1}^{3}}{2}\left(\pi_{15} \frac{2 M_{z}}{\pi R^{4}} Y+\frac{n_{1}^{2} n_{2}^{2} \pi_{64}^{2} \frac{4 M_{z}^{2}}{\pi^{2} R^{8}} X^{2}}{n_{2}^{2}-n_{1}^{2}+n_{1}^{2} n_{2}^{2}\left(\pi_{15}-\pi_{25}\right) \frac{2 M_{z}}{\pi R^{4}} Y}\right) \\
\simeq n_{1}-\frac{n_{1}^{3}}{2} \pi_{15} \sigma_{13}=n_{1}-n_{1}^{3} \pi_{15} \frac{M_{z}}{\pi R^{4}} Y \\
n_{2}^{\prime}=n_{2}-\frac{n_{2}^{3}}{2}\left(\pi_{25} \sigma_{13}-\frac{n_{1}^{2} n_{2}^{2} \pi_{64}^{2} \sigma_{23}^{2}}{n_{2}^{2}-n_{1}^{2}+n_{1}^{2} n_{2}^{2}\left(\pi_{15}-\pi_{25}\right) \sigma_{13}}\right) \\
=n_{2}-\frac{n_{2}^{3}}{2}\left(\pi_{25} \frac{2 M_{z}}{\pi R^{4}} Y-\frac{n_{1}^{2} n_{64}^{2} \frac{4 M_{z}^{2}}{\pi^{2} R^{8}} X^{2}}{n_{2}^{2}-n_{1}^{2}+n_{1}^{2} n_{2}^{2}\left(\pi_{15}-\pi_{25}\right) \frac{2 M_{z}}{\pi R^{4}} Y}\right. \\
\simeq n_{2}-\frac{n_{2}^{3}}{2} \pi_{25} \sigma_{13}=n_{2}-n_{2}^{3} \pi_{25} \frac{M_{z}}{\pi R^{4}} Y\end{array}$ \\
\hline
\end{tabular}




\begin{tabular}{|c|c|}
\hline 4 & 5 \\
\hline $\begin{array}{l}\delta(\Delta n)_{13}=\frac{1}{2}\left(n_{3}^{3} \pi_{35}-n_{1}^{3} \pi_{15}\right) \sigma_{13} \\
+\frac{1}{2} \frac{\left(n_{1}^{3}+n_{3}^{3}\right) n_{1}^{2} n_{3}^{2} \pi_{55}^{2} \sigma_{13}^{2}}{n_{1}^{2}-n_{3}^{2}+n_{1}^{2} n_{3}^{2}\left(\pi_{35}-\pi_{15}\right) \sigma_{13}} \\
=\left(n_{3}^{3} \pi_{35}-n_{1}^{3} \pi_{15}\right) \frac{M_{z}}{\pi R^{4}} Y \\
+2 \frac{\left(n_{1}^{3}+n_{3}^{3}\right) n_{1}^{2} n_{3}^{2} \pi_{55}^{2} \frac{M_{z}^{2}}{\pi^{2} R^{8}} Y^{2}}{n_{1}^{2}-n_{3}^{2}+n_{1}^{2} n_{3}^{2}\left(\pi_{35}-\pi_{15}\right) \frac{2 M_{z}}{\pi R^{4}} Y} \\
\simeq \frac{1}{2}\left(n_{3}^{3} \pi_{35}-n_{1}^{3} \pi_{15}\right) \sigma_{13} \\
=\left(n_{3}^{3} \pi_{35}-n_{1}^{3} \pi_{15}\right) \frac{M_{z}}{\pi R^{4}} Y\end{array}$ & $\begin{array}{l}\tan 2 \zeta_{Y}=\frac{2 n_{1}^{2} n_{3}^{2} \pi_{55} \sigma_{13}}{n_{1}^{2}-n_{3}^{2}+n_{1}^{2} n_{3}^{2}\left(\pi_{35}-\pi_{15}\right) \sigma_{13}} \\
=\frac{4 n_{1}^{2} n_{3}^{2} \pi_{55} \frac{M_{z}}{\pi R^{4}} Y}{n_{1}^{2}-n_{3}^{2}+n_{1}^{2} n_{3}^{2}\left(\pi_{35}-\pi_{15}\right) \frac{2 M_{z}}{\pi R^{4}} Y}\end{array}$ \\
\hline $\begin{array}{l}\delta(\Delta n)_{12}=\frac{1}{2}\left(n_{2}^{3} \pi_{25}-n_{1}^{3} \pi_{15}\right) \sigma_{13} \\
-\frac{1}{2} \frac{\left(n_{1}^{3}+n_{2}^{3}\right) n_{1}^{2} n_{2}^{2} \pi_{64}^{2} \sigma_{23}^{2}}{n_{2}^{2}-n_{1}^{2}+n_{1}^{2} n_{2}^{2}\left(\pi_{15}-\pi_{25}\right) \sigma_{13}} \\
=\left(n_{2}^{3} \pi_{25}-n_{1}^{3} \pi_{15}\right) \frac{M_{z}}{\pi R^{4}} Y \\
-2 \frac{\left(n_{1}^{3}+n_{2}^{3}\right) n_{1}^{2} n_{2}^{2} \pi_{64}^{2} \frac{M_{z}^{2}}{\pi^{2} R^{8}} X^{2}}{n_{2}^{2}-n_{1}^{2}+n_{1}^{2} n_{2}^{2}\left(\pi_{15}-\pi_{25}\right) \frac{2 M_{z}}{\pi R^{4}} Y} \\
\simeq \frac{1}{2}\left(n_{2}^{3} \pi_{25}-n_{1}^{3} \pi_{15}\right) \sigma_{13} \\
=\left(n_{2}^{3} \pi_{25}-n_{1}^{3} \pi_{15}\right) \frac{M_{z}}{\pi R^{4}} Y\end{array}$ & $\begin{array}{l}\tan 2 \zeta_{Z}=\frac{2 n_{1}^{2} n_{2}^{2} \pi_{64} \sigma_{23}}{n_{2}^{2}-n_{1}^{2}+n_{1}^{2} n_{2}^{2}\left(\pi_{15}-\pi_{25}\right) \sigma_{13}} \\
=\frac{4 n_{1}^{2} n_{2}^{2} \pi_{64} \frac{M_{z}}{\pi R^{4}} X}{n_{2}^{2}-n_{1}^{2}+n_{1}^{2} n_{2}^{2}\left(\pi_{15}-\pi_{25}\right) \frac{2 M_{z}}{\pi R^{4}} Y}\end{array}$ \\
\hline
\end{tabular}


Table 9. Changes in the optical indicatrix parameters under the torsion moment applied in crystals of the point symmetry groups 1 and $\overline{1}: M_{x}, \sigma_{12}, \sigma_{13}, k \| X$

\begin{tabular}{|c|c|}
\hline & 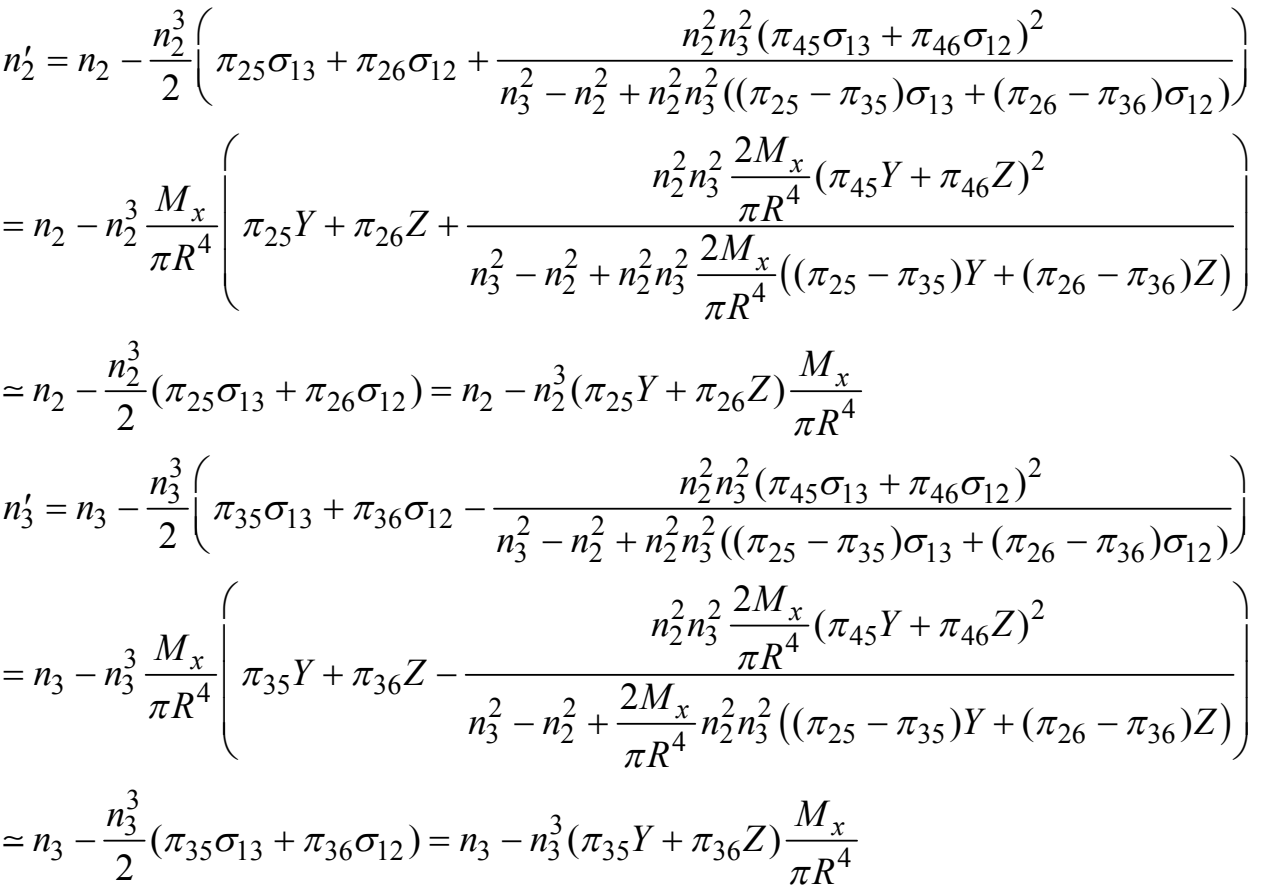 \\
\hline & 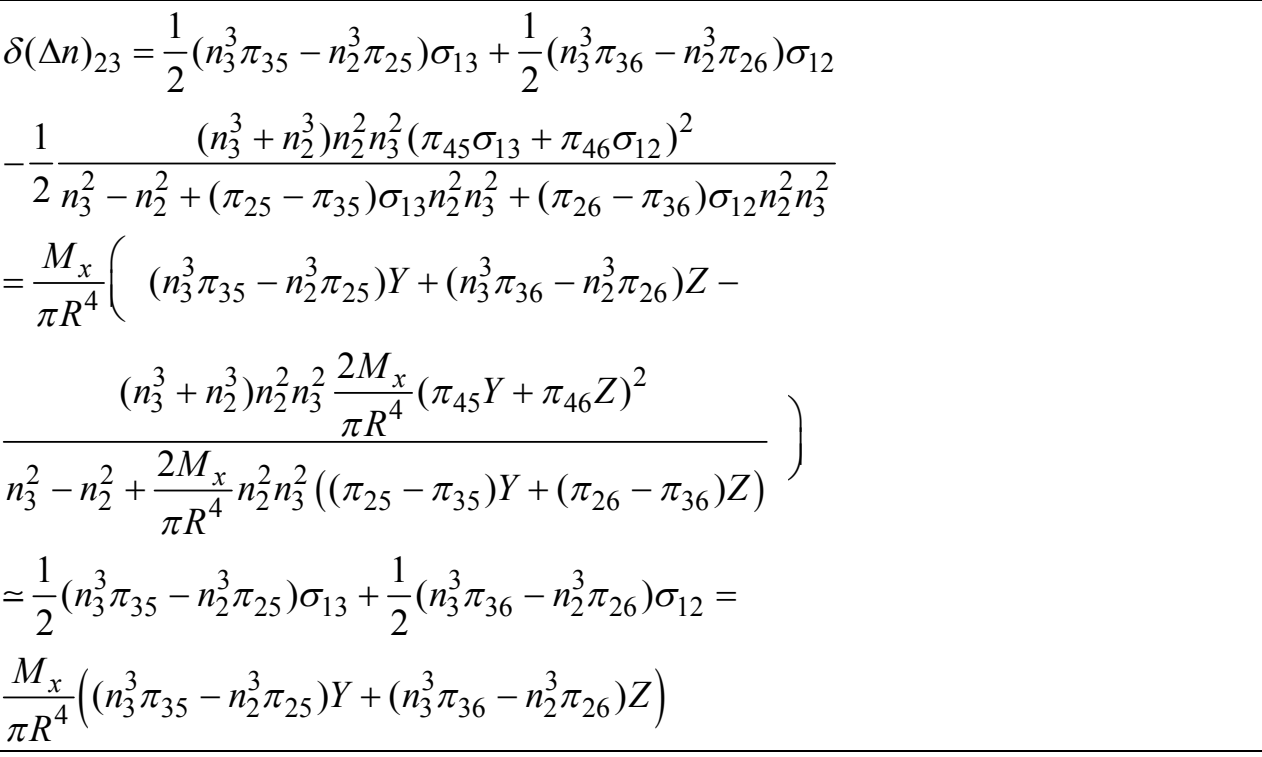 \\
\hline 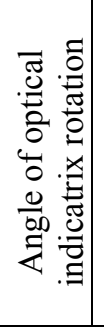 & $\begin{array}{l}\tan 2 \zeta_{X}=\frac{2 n_{2}^{2} n_{3}^{2}\left(\pi_{45} \sigma_{13}+\pi_{46} \sigma_{12}\right)}{n_{2}^{2}-n_{3}^{2}+n_{2}^{2} n_{3}^{2}\left(\pi_{25}-\pi_{35}\right) \sigma_{13}+n_{2}^{2} n_{3}^{2}\left(\pi_{26}-\pi_{36}\right) \sigma_{12}} \\
=\frac{4 n_{2}^{2} n_{3}^{2} \frac{M_{x}}{\pi R^{4}}\left(\pi_{45} Y+\pi_{46} Z\right)}{n_{2}^{2}-n_{3}^{2}+n_{2}^{2} n_{3}^{2} \frac{2 M_{x}}{\pi R^{4}}\left(\left(\pi_{25}-\pi_{35}\right) Y+\left(\pi_{26}-\pi_{36}\right) Z\right)}\end{array}$ \\
\hline
\end{tabular}


Torsion moment $-M_{x}$, stress tensor components $-\sigma_{12}, \sigma_{13}$ and direction of light propagation- $k \| Y$

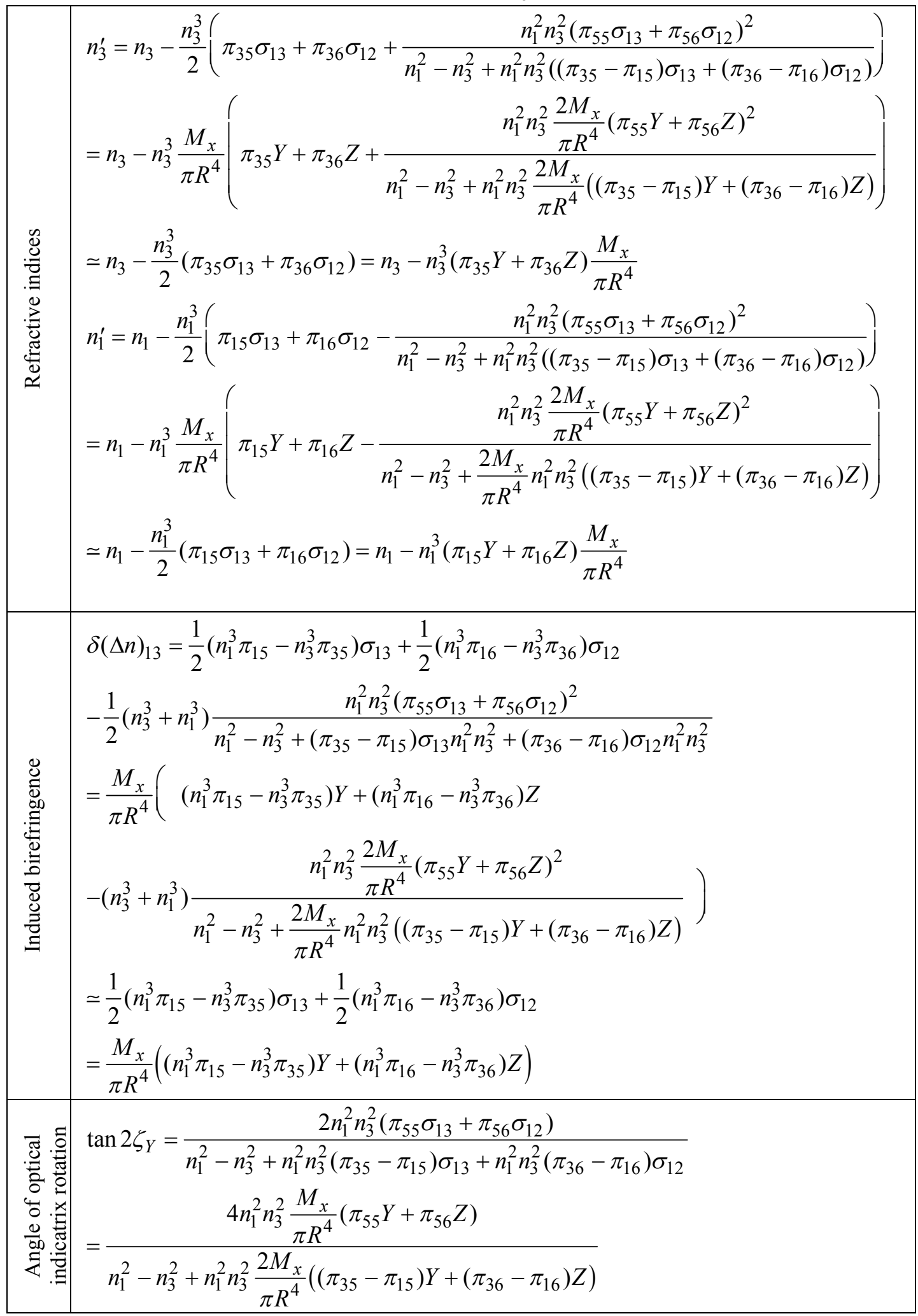


Torsion moment $-M_{x}$, stress tensor components $-\sigma_{12}, \sigma_{13}$ and direction of light propagation $-k \| Z$

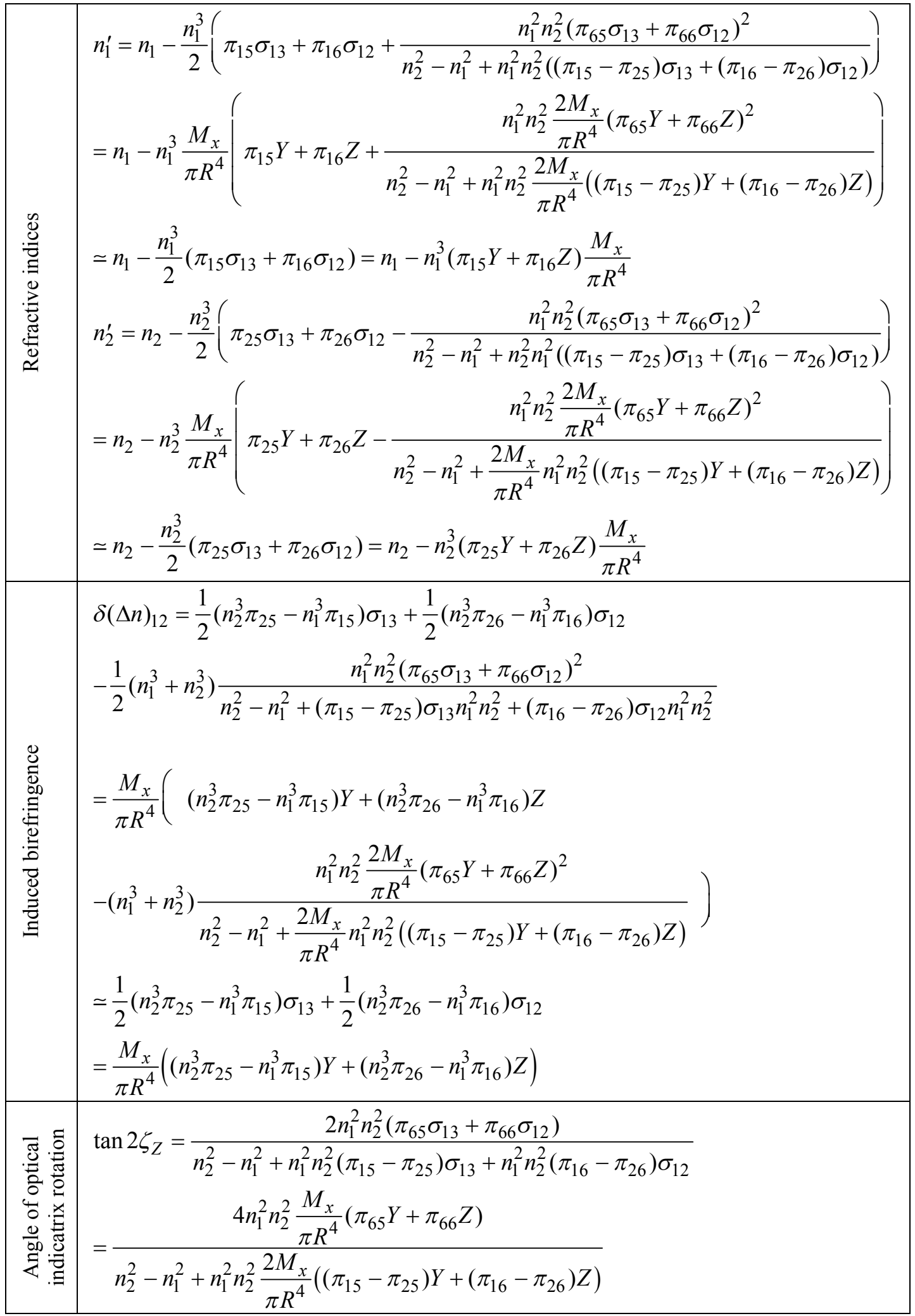


Torsion moment - $M_{y}$, stress tensor components- $\sigma_{12}, \sigma_{23}$ and direction of light propagation- $k \| X$

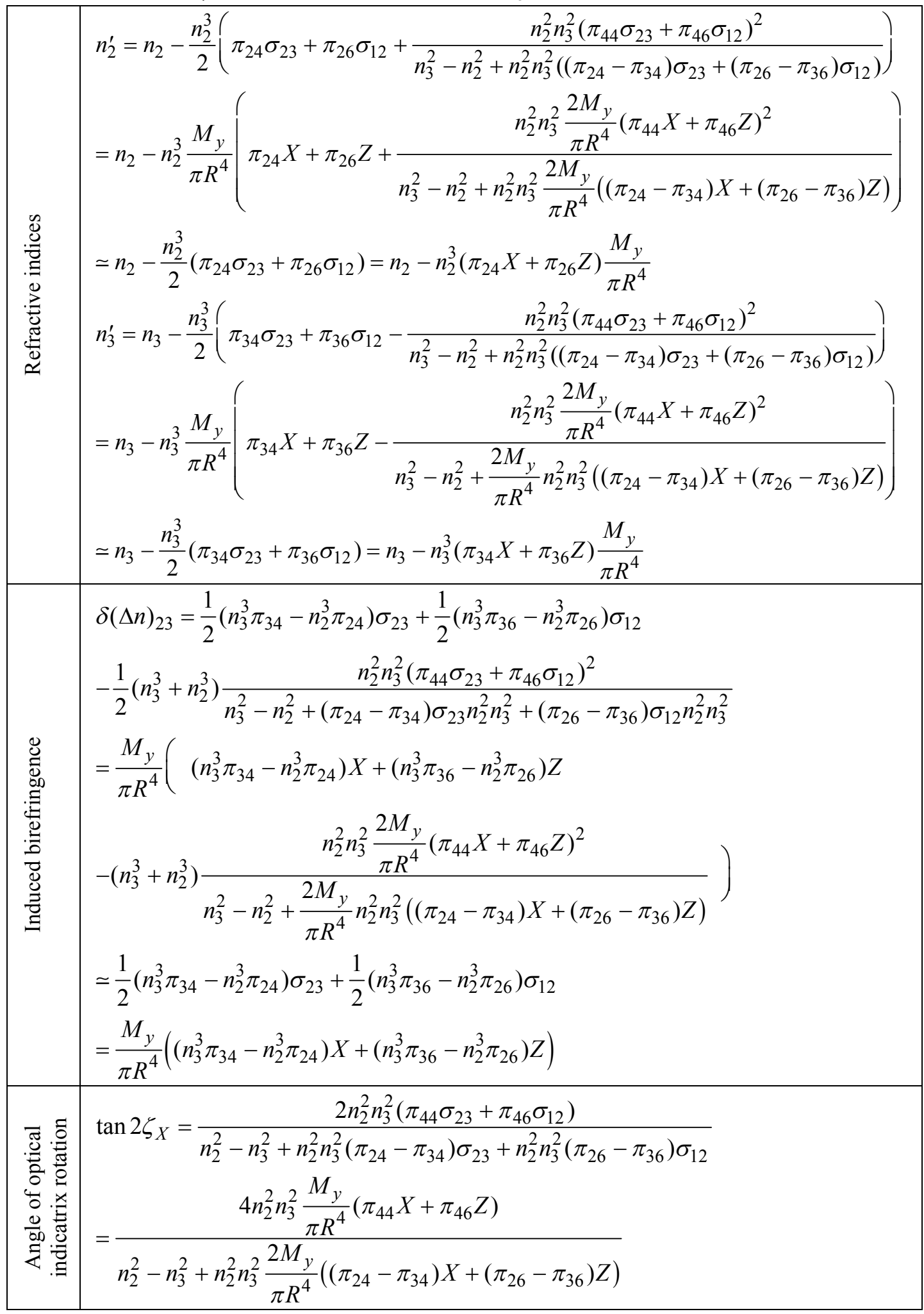


Torsion moment $-M_{y}$, stress tensor components- $\sigma_{12}, \sigma_{23}$ and direction of light propagation- $k \| Y$

\begin{tabular}{|c|c|}
\hline & $\begin{array}{l}n_{3}^{\prime}=n_{3}-\frac{n_{3}^{3}}{2}\left(\pi_{34} \sigma_{23}+\pi_{36} \sigma_{12}+\frac{n_{1}^{2} n_{3}^{2}\left(\pi_{54} \sigma_{23}+\pi_{56} \sigma_{12}\right)^{2}}{n_{1}^{2}-n_{3}^{2}+n_{1}^{2} n_{3}^{2}\left(\left(\pi_{34}-\pi_{14}\right) \sigma_{23}+\left(\pi_{36}-\pi_{16}\right) \sigma_{12}\right)}\right) \\
=n_{3}-n_{3}^{3} \frac{M_{y}}{\pi R^{4}}\left(\pi_{34} X+\pi_{36} Z+\frac{n_{3}^{2} \frac{2 M_{y}}{\pi R^{4}}\left(\pi_{54} X+\pi_{56} Z\right)^{2}}{n_{1}^{2}-n_{3}^{2}+n_{1}^{2} n_{3}^{2} \frac{2 M_{y}}{\pi R^{4}}\left(\left(\pi_{34}-\pi_{14}\right) X+\left(\pi_{36}-\pi_{16}\right) Z\right)}\right) \\
\simeq n_{3}-\frac{n_{3}^{3}}{2}\left(\pi_{34} \sigma_{23}+\pi_{36} \sigma_{12}\right)=n_{3}-n_{3}^{3}\left(\pi_{34} X+\pi_{36} Z\right) \frac{M_{y}}{\pi R^{4}} \\
n_{1}^{\prime}=n_{1}-\frac{n_{1}^{3}}{2}\left(\pi_{14} \sigma_{23}+\pi_{16} \sigma_{12}-\frac{n_{1}^{2} n_{3}^{2}\left(\pi_{54} \sigma_{23}+\pi_{56} \sigma_{12}\right)^{2}}{n_{1}^{2}-n_{3}^{2}+n_{1}^{2} n_{3}^{2}\left(\left(\pi_{34}-\pi_{14}\right) \sigma_{23}+\left(\pi_{36}-\pi_{16}\right) \sigma_{12}\right)}\right) \\
=n_{1}-n_{1}^{3} \frac{n_{1}^{2} n_{3}^{2} \frac{2 M_{y}}{\pi R^{4}}\left(\pi_{54} X+\pi_{56} Z\right)^{2}}{\pi R^{4}}\left(\pi_{14} X+\pi_{16} Z-\frac{\pi_{y}}{n_{1}^{2}-n_{3}^{2}+\frac{2 M_{y}}{\pi R^{4}} n_{1}^{2} n_{3}^{2}\left(\left(\pi_{34}-\pi_{14}\right) X+\left(\pi_{36}-\pi_{16}\right) Z\right)}\right) \\
\simeq n_{1}-\frac{n_{1}^{3}}{2}\left(\pi_{14} \sigma_{23}+\pi_{16} \sigma_{12}\right)=n_{1}-n_{1}^{3}\left(\pi_{14} X+\pi_{16} Z\right) \frac{M}{\pi R^{4}}\end{array}$ \\
\hline & $\begin{array}{l}\delta(\Delta n)_{13}=\frac{1}{2}\left(n_{1}^{3} \pi_{14}-n_{3}^{3} \pi_{34}\right) \sigma_{23}+\frac{1}{2}\left(n_{1}^{3} \pi_{16}-n_{3}^{3} \pi_{36}\right) \sigma_{12} \\
-\frac{1}{2}\left(n_{3}^{3}+n_{1}^{3}\right) \frac{n_{1}^{2} n_{3}^{2}\left(\pi_{54} \sigma_{23}+\pi_{56} \sigma_{12}\right)^{2}}{n_{1}^{2}-n_{3}^{2}+\left(\pi_{34}-\pi_{14}\right) \sigma_{23} n_{1}^{2} n_{3}^{2}+\left(\pi_{36}-\pi_{16}\right) \sigma_{12} n_{1}^{2} n_{3}^{2}} \\
=\frac{M_{y}}{\pi R^{4}}\left(n_{1}^{3} \pi_{14}-n_{3}^{3} \pi_{34}\right) X+\left(n_{1}^{3} \pi_{16}-n_{3}^{3} \pi_{36}\right) Z \\
\left.-\left(n_{3}^{3}+n_{1}^{3}\right) \frac{n_{1}^{2} n_{3}^{2} \frac{2 M_{y}}{\pi R^{4}}\left(\pi_{54} X+\pi_{56} Z\right)^{2}}{n_{1}^{2}-n_{3}^{2}+\frac{2 M_{y}}{\pi R^{4}} n_{1}^{2} n_{3}^{2}\left(\left(\pi_{34}-\pi_{14}\right) X+\left(\pi_{36}-\pi_{16}\right) Z\right)}\right) \\
\simeq \frac{1}{2}\left(n_{1}^{3} \pi_{14}-n_{3}^{3} \pi_{34}\right) \sigma_{23}+\frac{1}{2}\left(n_{1}^{3} \pi_{16}-n_{3}^{3} \pi_{36}\right) \sigma_{12} \\
=\frac{M_{y}}{\pi R^{4}}\left(\left(n_{1}^{3} \pi_{14}-n_{3}^{3} \pi_{34}\right) X+\left(n_{1}^{3} \pi_{16}-n_{3}^{3} \pi_{36}\right) Z\right)\end{array}$ \\
\hline 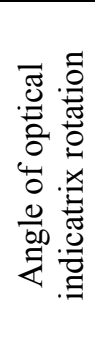 & $\begin{array}{l}\tan 2 \zeta_{Y}=\frac{2 n_{1}^{2} n_{3}^{2}\left(\pi_{54} \sigma_{23}+\pi_{56} \sigma_{12}\right)}{n_{1}^{2}-n_{3}^{2}+n_{1}^{2} n_{3}^{2}\left(\pi_{34}-\pi_{14}\right) \sigma_{23}+n_{1}^{2} n_{3}^{2}\left(\pi_{36}-\pi_{16}\right) \sigma_{12}} \\
=\frac{4 n_{1}^{2} n_{3}^{2} \frac{M_{y}}{\pi R^{4}}\left(\pi_{54} X+\pi_{56} Z\right)}{n_{1}^{2}-n_{3}^{2}+n_{1}^{2} n_{3}^{2} \frac{2 M_{y}}{\pi R^{4}}\left(\left(\pi_{34}-\pi_{14}\right) X+\left(\pi_{36}-\pi_{16}\right) Z\right)}\end{array}$ \\
\hline
\end{tabular}


Torsion moment $-M_{y}$, stress tensor components- $\sigma_{12}, \sigma_{23}$ and direction of light propagation - $k \| Z$

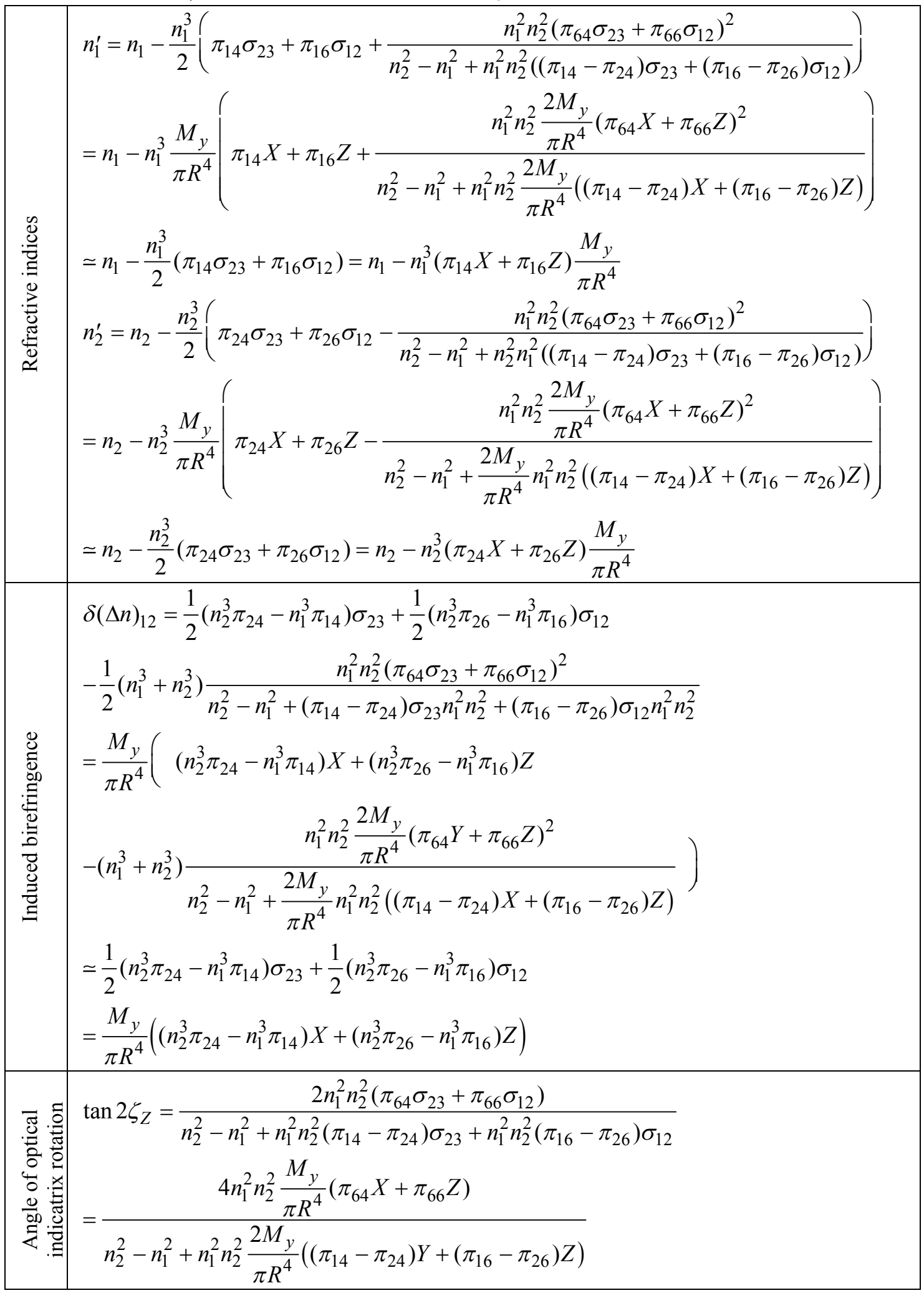


Torsion moment- $M_{z}$, stress tensor components- $\sigma_{13}, \sigma_{23}$ and direction of light propagation- $k \| X$

\begin{tabular}{|c|c|}
\hline & $\begin{array}{l}n_{2}^{\prime}=n_{2}-\frac{n_{2}^{3}}{2}\left(\pi_{24} \sigma_{23}+\pi_{25} \sigma_{13}+\frac{n_{2}^{2} n_{3}^{2}\left(\pi_{44} \sigma_{23}+\pi_{45} \sigma_{13}\right)^{2}}{n_{3}^{2}-n_{2}^{2}+n_{2}^{2} n_{3}^{2}\left(\left(\pi_{24}-\pi_{34}\right) \sigma_{23}+\left(\pi_{25}-\pi_{35}\right) \sigma_{13}\right)}\right) \\
=n_{2}-n_{2}^{3} \frac{M_{z}}{\pi R^{4}}\left(\pi_{24} X+\pi_{25}^{2} Y+\frac{n_{3}^{2} \frac{2 M_{z}}{\pi R^{4}}\left(\pi_{44} X+\pi_{45} Y\right)^{2}}{n_{3}^{2}-n_{2}^{2}+n_{2}^{2} n_{3}^{2} \frac{2 M_{z}}{\pi R^{4}}\left(\left(\pi_{24}-\pi_{34}\right) X+\left(\pi_{25}-\pi_{35}\right) Y\right)}\right) \\
\simeq n_{2}-\frac{n_{2}^{3}}{2}\left(\pi_{24} \sigma_{23}+\pi_{25} \sigma_{13}\right)=n_{2}-n_{2}^{3}\left(\pi_{24} X+\pi_{25} Y\right) \frac{M_{z}}{\pi R^{4}} \\
n_{3}^{\prime}=n_{3}-\frac{n_{3}^{3}}{2}\left(\pi_{34} \sigma_{23}+\pi_{35} \sigma_{13}-\frac{n_{2}^{2} n_{3}^{2}\left(\pi_{44} \sigma_{23}+\pi_{45} \sigma_{13}\right)^{2}}{n_{3}^{2}-n_{2}^{2}+n_{2}^{2} n_{3}^{2}\left(\left(\pi_{24}-\pi_{34}\right) \sigma_{23}+\left(\pi_{25}-\pi_{35}\right) \sigma_{13}\right)}\right) \\
=n_{3}-n_{3}^{3} \frac{n_{3}^{2} \frac{2 n_{z}}{\pi R^{4}}\left(\pi_{44} X+\pi_{45} Y\right)^{2}}{\pi R^{4}}\left(\pi_{34} X+\pi_{35} Y-\frac{\pi_{z}}{n_{3}^{2}-n_{2}^{2}+\frac{2 M_{z}}{\pi R^{4}} n_{2}^{2} n_{3}^{2}\left(\left(\pi_{24}-\pi_{34}\right) X+\left(\pi_{25}-\pi_{35}\right) Y\right)}\right) \\
\simeq n_{3}-\frac{n_{3}^{3}}{2}\left(\pi_{34} \sigma_{23}+\pi_{35} \sigma_{13}\right)=n_{3}-n_{3}^{3}\left(\pi_{34} X+\pi_{35} Y\right) \frac{M_{z}}{\pi R^{4}}\end{array}$ \\
\hline & $\begin{array}{l}\delta(\Delta n)_{23}=\frac{1}{2}\left(n_{3}^{3} \pi_{34}-n_{2}^{3} \pi_{24}\right) \sigma_{23}+\frac{1}{2}\left(n_{3}^{3} \pi_{35}-n_{2}^{3} \pi_{25}\right) \sigma_{13} \\
-\frac{1}{2}\left(n_{3}^{3}+n_{2}^{3}\right) \frac{n_{2}^{2} n_{3}^{2}\left(\pi_{44} \sigma_{23}+\pi_{45} \sigma_{13}\right)^{2}}{n_{3}^{2}-n_{2}^{2}+\left(\pi_{24}-\pi_{34}\right) \sigma_{23} n_{2}^{2} n_{3}^{2}+\left(\pi_{25}-\pi_{35}\right) \sigma_{13} n_{2}^{2} n_{3}^{2}} \\
=\frac{M_{z}}{\pi R^{4}}\left(\left(n_{3}^{3} \pi_{34}-n_{2}^{3} \pi_{24}\right) X+\left(n_{3}^{3} \pi_{35}-n_{2}^{3} \pi_{25}\right) Y\right. \\
\left.-\left(n_{3}^{3}+n_{2}^{3}\right) \frac{n_{2}^{2} n_{3}^{2} \frac{2 M_{z}}{\pi R^{4}}\left(\pi_{44} X+\pi_{45} Y\right)^{2}}{n_{3}^{2}-n_{2}^{2}+\frac{2 M_{z}}{\pi R^{4}} n_{2}^{2} n_{3}^{2}\left(\left(\pi_{24}-\pi_{34}\right) X+\left(\pi_{25}-\pi_{35}\right) Y\right)}\right) \\
\simeq \frac{1}{2}\left(n_{3}^{3} \pi_{34}-n_{2}^{3} \pi_{24}\right) \sigma_{23}+\frac{1}{2}\left(n_{3}^{3} \pi_{35}-n_{2}^{3} \pi_{25}\right) \sigma_{13} \\
=\frac{M_{z}}{\pi R^{4}}\left(\left(n_{3}^{3} \pi_{34}-n_{2}^{3} \pi_{24}\right) X+\left(n_{3}^{3} \pi_{35}-n_{2}^{3} \pi_{25}\right) Y\right)\end{array}$ \\
\hline 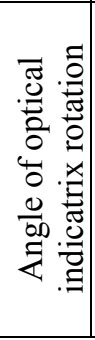 & $\begin{array}{l}\tan 2 \zeta_{X}=\frac{2 n_{2}^{2} n_{3}^{2}\left(\pi_{44} \sigma_{23}+\pi_{45} \sigma_{13}\right)}{n_{2}^{2}-n_{3}^{2}+n_{2}^{2} n_{3}^{2}\left(\pi_{24}-\pi_{34}\right) \sigma_{23}+n_{2}^{2} n_{3}^{2}\left(\pi_{25}-\pi_{35}\right) \sigma_{13}} \\
=\frac{4 n_{2}^{2} n_{3}^{2} \frac{M_{z}}{\pi R^{4}}\left(\pi_{44} X+\pi_{45} Y\right)}{n_{2}^{2}-n_{3}^{2}+n_{2}^{2} n_{3}^{2} \frac{2 M_{z}}{\pi R^{4}}\left(\left(\pi_{24}-\pi_{34}\right) X+\left(\pi_{25}-\pi_{35}\right) Y\right)}\end{array}$ \\
\hline
\end{tabular}


Torsion moment $-M_{z}$, stress tensor components- $\sigma_{13}, \sigma_{23}$ and direction of light propagation - $k \| Y$

\begin{tabular}{|c|c|}
\hline 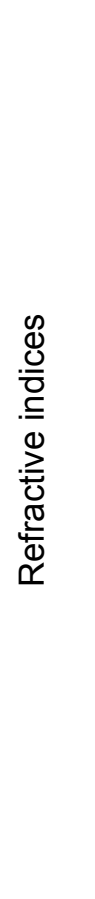 & 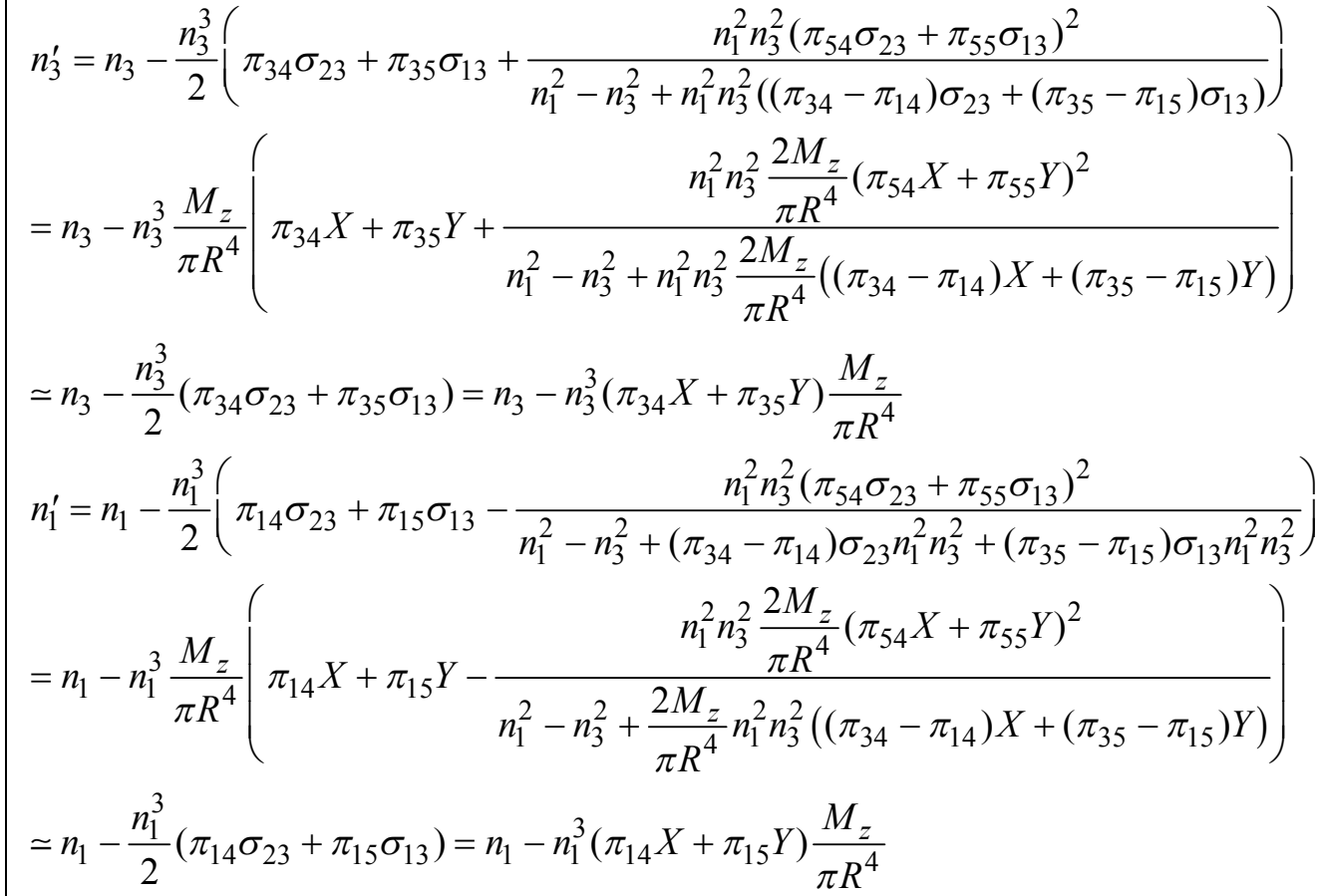 \\
\hline & $\begin{array}{l}\delta(\Delta n)_{13}=\frac{1}{2}\left(n_{1}^{3} \pi_{14}-n_{3}^{3} \pi_{34}\right) \sigma_{23}+\frac{1}{2}\left(n_{1}^{3} \pi_{15}-n_{3}^{3} \pi_{35}\right) \sigma_{13} \\
-\frac{1}{2}\left(n_{3}^{3}+n_{1}^{3}\right) \frac{n_{1}^{2} n_{3}^{2}\left(\pi_{54} \sigma_{23}+\pi_{55} \sigma_{13}\right)^{2}}{n_{1}^{2}-n_{3}^{2}+\left(\pi_{34}-\pi_{14}\right) \sigma_{23} n_{1}^{2} n_{3}^{2}+\left(\pi_{35}-\pi_{15}\right) \sigma_{13} n_{1}^{2} n_{3}^{2}} \\
=\frac{M_{z}}{\pi R^{4}}\left(\left(n_{1}^{3} \pi_{14}-n_{3}^{3} \pi_{34}\right) X+\left(n_{1}^{3} \pi_{15}-n_{3}^{3} \pi_{35}\right) Y\right. \\
\left.-\left(n_{3}^{3}+n_{1}^{3}\right) \frac{n_{1}^{2} n_{3}^{2} \frac{2 M_{z}}{\pi R^{4}}\left(\pi_{54} X+\pi_{55} Y\right)^{2}}{n_{1}^{2}-n_{3}^{2}+\frac{2 M_{z}}{\pi R^{4}} n_{1}^{2} n_{3}^{2}\left(\left(\pi_{34}-\pi_{14}\right) X+\left(\pi_{35}-\pi_{15}\right) Y\right)}\right) \\
\simeq \frac{1}{2}\left(n_{1}^{3} \pi_{14}-n_{3}^{3} \pi_{34}\right) \sigma_{23}+\frac{1}{2}\left(n_{1}^{3} \pi_{15}-n_{3}^{3} \pi_{35}\right) \sigma_{13} \\
=\frac{M_{z}}{\pi R^{4}}\left(\left(n_{1}^{3} \pi_{14}-n_{3}^{3} \pi_{34}\right) X+\left(n_{1}^{3} \pi_{15}-n_{3}^{3} \pi_{35}\right) Y\right)\end{array}$ \\
\hline 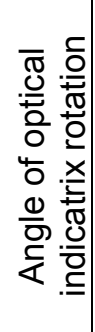 & $\begin{array}{l}\tan 2 \zeta_{Y}=\frac{2 n_{1}^{2} n_{3}^{2}\left(\pi_{54} \sigma_{23}+\pi_{55} \sigma_{13}\right)}{n_{1}^{2}-n_{3}^{2}+n_{1}^{2} n_{3}^{2}\left(\pi_{34}-\pi_{14}\right) \sigma_{23}+n_{1}^{2} n_{3}^{2}\left(\pi_{35}-\pi_{15}\right) \sigma_{13}} \\
=\frac{4 n_{1}^{2} n_{3}^{2} \frac{M_{z}}{\pi R^{4}}\left(\pi_{54} X+\pi_{55} Y\right)}{n_{1}^{2}-n_{3}^{2}+n_{1}^{2} n_{3}^{2} \frac{2 M_{z}}{\pi R^{4}}\left(\left(\pi_{34}-\pi_{14}\right) X+\left(\pi_{35}-\pi_{15}\right) Y\right)}\end{array}$ \\
\hline
\end{tabular}


Torsion moment- $M_{z}$, stress tensor components- $\sigma_{13}, \sigma_{23}$ and direction of light propagation- $k \| Z$

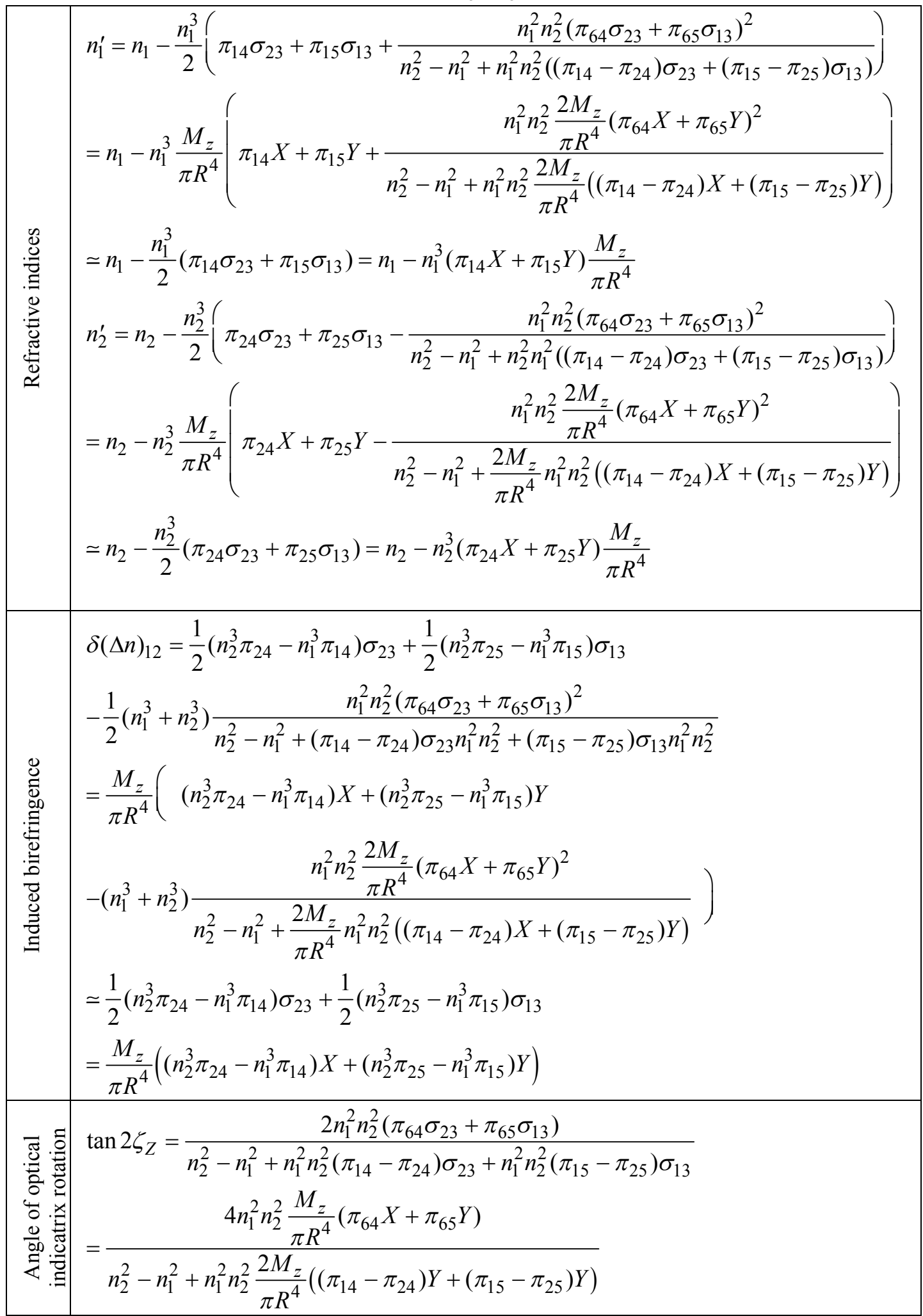


accompanied by additional normal displacements in any other geometry of sample loading, thus leading to appearance of the compression and/or extension stress components. As a matter of fact, this is one of the reasons why complicated relations appear that couple so many piezooptic tensor components with the mechanical stresses. This is also a clear reason for increasing error of determination of a particular piezooptic coefficient.

In spite of promises associated with the piezooptic experiments that use torsion loading, the relations for the optical indicatrix perturbed by the torsion in crystals have not yet been derived. As a result, the goal of the present work is to deduce theoretical relations for the refractive indices, the birefringence and the optical indicatrix rotation describing the torsion of crystals belonging to different point groups of symmetry.

\section{Results}

In general, the piezooptic tensor may be presented as

\begin{tabular}{l|llllll} 
& $\sigma_{11}$ & $\sigma_{22}$ & $\sigma_{33}$ & $\sigma_{32}$ & $\sigma_{31}$ & $\sigma_{21}$ \\
\hline$\Delta B_{11}$ & $\pi_{11}$ & $\pi_{12}$ & $\pi_{13}$ & $\pi_{14}$ & $\pi_{15}$ & $\pi_{16}$ \\
$\Delta B_{22}$ & $\pi_{21}$ & $\pi_{22}$ & $\pi_{23}$ & $\pi_{24}$ & $\pi_{25}$ & $\pi_{26}$ \\
$\Delta B_{33}$ & $\pi_{31}$ & $\pi_{32}$ & $\pi_{33}$ & $\pi_{34}$ & $\pi_{35}$ & $\pi_{36}$ \\
$\Delta B_{32}$ & $\pi_{41}$ & $\pi_{42}$ & $\pi_{43}$ & $\pi_{44}$ & $\pi_{45}$ & $\pi_{46}$ \\
$\Delta B_{31}$ & $\pi_{51}$ & $\pi_{52}$ & $\pi_{53}$ & $\pi_{54}$ & $\pi_{55}$ & $\pi_{56}$ \\
$\Delta B_{21}$ & $\pi_{61}$ & $\pi_{62}$ & $\pi_{63}$ & $\pi_{64}$ & $\pi_{65}$ & $\pi_{66}$
\end{tabular}

The piezooptic coefficients under our interest are indicated by the blue colour. The general form of equation for the optical indicatrix subjected to the torsions around the $X$, $Y$ and $Z$ axes (the torque moments $M_{x}, M_{y}$ and $M_{z}$, respectively) is as follows:

$$
B_{11} X^{2}+B_{22} Y^{2}+B_{33} Z^{2}+2 B_{23} Y Z+2 B_{13} X Z+2 B_{12} X Z=1,
$$

where $B_{i j}$ denote the coefficients depending upon the stress tensor components and, subsequently, on the torque moments.

The optical indicatrix parameters may be derived basing on eigen values of the optical impermeability tensor for different cross sections perpendicular to the light wave vector direction. The principal refractive indices, the optical birefringence and the angles of the optical indicatrix rotation thus obtained by us for the crystals and textures of different symmetry systems are presented in Tables 1 to 9 .

\section{Conclusion}

In the present work we have derived the relations that describe the optical indicatrix changes appearing for all of the point symmetry groups for different cases of geometries concerned with the torque moment application and the light propagation. The aim of this study has not included a comprehensive analysis of the relations presented above, so that 
the paper has mainly a systematic value. A detailed analysis should be performed separately for each specific experimental situation.

\section{References}

1. Narasimhamurty T S, Photoelastic and electro-optic properties of crystals. New York: Plenum, (1981).

2. Mytsyk B, 2003. Methods for the studies of the piezo-optical effect in crystals and the analysis of experimental data. I. Methodology for the studies of piezo-optical effect. Ukr. J. Phys. Opt. 4: 1-26.

3. Mytsyk B, 2003. Methods for the studies of the piezo-optical effect in crystals and the analysis of experimental data. II. Analysis of experimental data. Ukr. J. Phys. Opt. 4: $105-118$.

4. Vasylkiv $\mathrm{Yu}$, Kvasnyuk $\mathrm{O}$, Krupych $\mathrm{O}$, Mys $\mathrm{O}$, Maksymuk $\mathrm{O}$ and Vlokh $\mathrm{R}$, Reconstruction of 3D stress fields basing on piezooptic experiment. 2009. Ukr. J. Phys. Opt. 10: 22-37.

5. Peng H J, Wong S P and Ho H P, 2004. Measurement of orientation dependent stressoptic coefficient of GaAs single crystals. Appl. Phys. Lett. 84: 1829-1831.

6. Vlokh RO, Pyatak YA and Skab IP, 1991. The elasto-optic effect in $\mathrm{LiNbO}_{3}$ crystals under the torsion. Fiz. Tverd. Tela. 33: 2467-2470.

7. Vlokh R, Kostyrko M and Skab I, 1998. Principle and application of crystallo-optical effects induced by inhomogeneous deformation. Japan. J. Appl. Phys. 37: 5418-5420.

8. Vlokh R, Pyatak Y and Skab I, 1992. Elasto-optic effect in $\mathrm{LiNbO}_{3}$ under the crystal bending. Ferroelectrics. 126: 239-242.

9. Vlokh R, Kostyrko M and Skab I, 1997. The observation of "neutral" birefringence line in $\mathrm{LiNbO}_{3}$ crystals under torsion. Ferroelectrics. 203: 113-117.

10. Vlokh R O, Kostyrko M E and Skab I P, 1997. Description of gradients of piezogyration and piezo-optics caused by twisting and bending. Crystallogr. Rep. 42: 1011-1013.

11. Sirotin Yu I and Shaskolskaya M P. Fundamentals of crystal physics. Moscow: Nauka (1979).

Skab I., Vasylkiv Yu., Savaryn V. and Vlokh R., 2010. Relations for optical indicatrix parameters in the conditions of crystal torsion. Ukr.J.Phys.Opt. 11: 193-240.

Анотація. В роботі отримані співвідношення, які описують зміни оптичних індикатрис в кристалах всіх точкових груп симетрії при різних геометріях прикладання торсійного моменту і напрямках поширення світла. 